\title{
Brain
}

\section{Alpha-synuclein targets GluN2A NMDA receptor subunit causing striatal synaptic dysfunction and visuo-spatial memory alteration}

\begin{tabular}{|c|c|}
\hline Journal: & Brain \\
\hline Manuscript ID & BRAIN-2018-00774.R2 \\
\hline Manuscript Type: & Original Article \\
\hline $\begin{array}{r}\text { Date Submitted by the } \\
\text { Author: }\end{array}$ & 07-Jan-2019 \\
\hline Complete List of Authors: & $\begin{array}{l}\text { Durante, Valentina; Università degli Studi di Perugia } \\
\text { de Iure, Antonio; Università degli Studi di Perugia, } \\
\text { Loffredo, Vittorio; La Sapienza University, PhD Program in Behavioral } \\
\text { Neuroscience } \\
\text { Vaikath, Nishant; Neurological Disorders Research Center, Qatar } \\
\text { Biomedical Research Institute (QBRI), Hamad Bin Khalifa University } \\
\text { (HBKU), Qatar Foundation, Doha, Qatar } \\
\text { De Risi, Maria; Telethon Institute of Genetics and Medicine (TIGEM), } \\
\text { Faculty and Head of the Behavioral Facility } \\
\text { Paciotti, Silvia; Universita degli Studi di Perugia, } \\
\text { Quiroga-Varela, Ana; Università degli Studi di Perugia Italy, } \\
\text { Chiasserini, Davide; Università di Perugia, Ospedale S. Maria della } \\
\text { Misericordia, Clinica Neurologica } \\
\text { Mellone, Manuela; University of Milan, Department of Pharmacological } \\
\text { Sciences } \\
\text { Mazzocchetti, Petra; Università degli Studi di Perugia } \\
\text { Calabrese, Valeria; Università degli Studi di Perugia Italy } \\
\text { Campanelli, Federica; Università degli Studi di Perugia Italy } \\
\text { Mechelli, Alessandro; Università degli Studi di Perugia } \\
\text { Di Filippo, Massimiliano; Università degli Studi di Perugia, } \\
\text { Ghiglieri, Veronica; Fondazione Santa Lucia, IRCCS, Neurophysiology } \\
\text { Picconi, Barbara; IRCCS, San Raffaele Pisana, Rome } \\
\text { El-Agnaf, Omar; Neurological Disorders Research Center, Qatar } \\
\text { Biomedical Research Institute (QBRI), Hamad Bin Khalifa University } \\
\text { (HBKU), Qatar Foundation, Doha, Qatar } \\
\text { De Leonibus, Elvira; Telethon Institute of Genetics and Medicine } \\
\text { (TIGEM), Faculty and Head of the Behavioral Facility } \\
\text { Gardoni, Fabrizio; University of Milan, Department of Pharmacological } \\
\text { Sciences } \\
\text { Tozzi, Alessandro; Università degli Studi di Perugia, Neurologia } \\
\text { Sperimentale } \\
\text { Calabresi, Paolo; Università di Perugia, Ospedale S. Maria della } \\
\text { Misericordia, Clinica Neurologica }\end{array}$ \\
\hline Subject category: & Movement disorders \\
\hline To search keyword list, use & Parkinson's disease < MOVEMENT DISORDERS, Parkinson's disease: \\
\hline
\end{tabular}


whole or part words followed neurophysiology < MOVEMENT DISORDERS, alpha-Synuclein < by an *: NEURODEGENERATION: CELLULAR AND MOLECULAR, Neurodegeneration: biomarkers < NEURODEGENERATION: CELLULAR AND MOLECULAR, Basal ganglia < MOVEMENT DISORDERS

\section{SCHOLARONE ${ }^{\text {M }}$ \\ Manuscripts}




\section{Alpha-synuclein targets GluN2A NMDA receptor subunit causing striatal synaptic dysfunction and visuo-spatial memory alteration}

Durante $\mathrm{V}^{1}$, de Iure $\mathrm{A}^{1,2}$, Loffredo $\mathrm{V}^{3,4}$, Vaikath $\mathrm{N}^{5}$, De Risi $\mathrm{M}^{6}$, Paciotti $\mathrm{S}^{7}$, Quiroga-Varela $\mathrm{A}^{1}$, Chiasserini $\mathrm{D}^{1}$, Mellone $\mathrm{M}^{8}$, Mazzocchetti $\mathrm{P}^{1}$, Calabrese $\mathrm{V}^{1}$, Campanelli $\mathrm{F}^{1,9}$, Mechelli $\mathrm{A}^{1}$, Di Filippo $\mathrm{M}^{1}$, Ghiglieri $\mathrm{V}^{9,10}$, Picconi $\mathrm{B}^{2}$, El-Agnaf $\mathrm{OM}^{5}$, De Leonibus E ${ }^{3,6}$, Gardoni $\mathrm{F}^{8}$, Tozzi $\mathrm{A}^{7,9}$, and Calabresi $\mathrm{P}^{1,9^{*}}$

1. Neurological Clinic, Department of Medicine, Hospital Santa Maria della Misericordia, University of Perugia, Perugia, Italy;

2. Laboratory of Experimental Neurophysiology, IRCCS San Raffaele Pisana, Rome, Italy

3. Institute of Cellular Biology and Neurobiology, National Research Council, Monterotondo (Rome), Italy;

4. PhD Program in Behavioral Neuroscience, Sapienza University of Rome, Italy;

5. Neurological Disorders Research Center, Qatar Biomedical Research Institute (QBRI), Hamad Bin Khalifa University (HBKU), Qatar Foundation, Doha, Qatar;

6. Telethon Institute of Genetics and Medicine, Telethon Foundation, Pozzuoli (NA), Italy;

7. Department of Experimental Medicine, Section of Physiology and Biochemistry, University of Perugia, Perugia, Italy;

8. Department of Pharmacological and Biomolecular Sciences, University of Milan, Italy;

9. Laboratory of Neurophysiology, Santa Lucia Foundation, IRCCS, Rome, Italy;

10. Department of Philosophy, Human, Social and Educational Sciences, University of Perugia, Perugia, Italy.

Correspondence to:

Professor Paolo Calabresi

Neurological Clinic, Department of Medicine, University of Perugia,

Hospital Santa Maria della Misericordia,

06132 - S. Andrea delle Fratte, Perugia, Italy

Phone +39-075-578-4230; Fax +39-075-578-4229

Email: paolo.calabresi@unipg.it

Running title: Alpha-synuclein in synaptic transmission 


\begin{abstract}
Parkinson's disease is a progressive neurodegenerative disorder characterized by altered striatal dopaminergic signalling that leads to motor and cognitive deficits. Parkinson's disease is also characterized by abnormal presence of soluble toxic forms of alpha-synuclein ( $\alpha$-syn) that, when clustered into Lewy bodies, represents one of the pathological hallmark of the disease. However, also $\alpha$-syn oligomers might directly affect synaptic transmission and plasticity in Parkinson's disease models. Accordingly, by combining electrophysiological, optogenetic, immunofluorescence, molecular and behavioural analyses, here we report that $\alpha$-syn reduces $\mathrm{N}$ methyl-D-aspartate (NMDA) receptor-mediated synaptic currents and impairs corticostriatal longterm potentiation (LTP) of striatal spiny projection neurons (SPNs), of both direct (D1-positive) and indirect (putative D2-positive) pathways. Intrastriatal injections of $\alpha$-syn produce deficits in visuospatial learning associated with reduced function of GluN2A NMDA receptor subunit indicating that this protein selectively targets this subunit both in vitro and ex vivo. Interestingly, this effect is observed in SPNs activated by optical stimulation of either cortical or thalamic glutamatergic afferents. We also found that treatment of striatal slices with antibodies targeting $\alpha$-syn prevents the $\alpha$-syn-induced loss of LTP and the reduced synaptic localization of GluN2A NMDA receptor subunit suggesting that this strategy might counteract synaptic dysfunction occurring in Parkinson's disease.
\end{abstract}

KEYWORDS: Dopamine; Glutamate; Long-term potentiation; Monoclonal antibodies; Oligomers; Optogenetics; Parkinson's disease; Synaptic plasticity; Striatum. 


\section{INTRODUCTION}

Alpha-synuclein ( $\alpha$-syn) is a 140 amino acids presynaptic protein causally involved in the pathogenesis of Parkinson's Disease (Spillantini and Goedert, 2016). The main mechanisms of $\alpha$ syn-mediated cellular damage involve mitochondrial function, endoplasmic reticulum-Golgi trafficking, calcium ions influx through formation of pore-like structures, proteins degradation and oxidative stress (Gallegos et al., 2015; Wong and Krainc, 2017).

The protein exists as unstructured monomer widely distributed in the central nervous system and is involved in the regulation of presynaptic vesicle pool, neurotransmitter release, synaptic function and plasticity (Abeliovich et al., 2000; Cabin et al., 2002). However, $\alpha$-syn monomers have the intrinsic tendency to aggregate in structures of higher molecular weights (Gallegos et al., 2015) and in particular conditions, such as $\mathrm{pH}$ variations, oxidative stress, mutations and overexpression of $\alpha$ syn gene. This process can lead to the formation of $\alpha$-syn oligomers, protofibrils and eventually fibrils, the main components of Lewy Bodies (LBs) (Spillantini et al., 1997; Wong and Krainc, 2017). While LBs represent a neuropathological hallmark of Parkinson's disease and the histological sign of synucleinopathies, they show no actual direct neurotoxic properties (Tanaka et al., 2004). Mechanisms mediating $\alpha$-syn toxicity in humans have not been determined yet, however, several studies point to $\alpha$-syn oligomers and to protofibrils as major players in synaptic dysfunction (Diogenes et al., 2012; Rockenstein et al., 2014).

The striatum, a subcortical nucleus receiving major excitatory inputs from the cortex and the thalamus (Ding et al., 2008), is a brain region particularly involved in Parkinson's disease and oligomeric forms of $\alpha$-syn may affect synaptic transmission and long-term synaptic plasticity in this structure, leading to the motor and behavioural deficits observed in Parkinson's disease (Tozzi et al., 2016). Alpha-synuclein oligomers may produce distinct effects on different striatal neuronat subtypes or synaptic sites. In fact, we recently provided evidence that long-term potentiation (LTP) 
of excitatory synaptic transmission of striatal cholinergic interneurons (ChIs) is impaired by a low nanomolar dose of exogenous human a-syn oligomers (Tozzi et al., 2016). In this previous study, we-found that the loss of LTP in striatal ChIs was induced by a direct effect of $\alpha$-syn on GluN2Dexpressing $\mathrm{N}$-methyl-D-aspartate receptors (NMDARs), which are selectively expressed in this class of interneurons.

Although in Parkinson's disease models pathological changes in synaptic plasticity have been widely reported in vivo as well as in vitro (Calabresi et al., 2014), at present, no data are available on the effects of $\alpha$-syn on synaptic transmission and synaptic plasticity of striatal spiny projection neurons (SPNs) and their possible underlying mechanisms.

SPNs are GABAergic output neurons, which represent the large majority of neuronal population in the striatum (Calabresi et al., 2014). They express a peculiar NMDA subunit receptor composition including GluN2A and GluN2B subunits (Dunah et al., 2000; Gardoni et al., 2006), whose distinct activation regulates glutamatergic inputs in striatal SPNs. In fact, the selective pharmacological modulation of these subunits differentially alters amplitude and kinetics of NMDARs responses also affecting responses to dopaminergic modulation (Jocoy et al., 2011; Vastagh et al., 2012). Moreover, an unbalanced GluN2A/GluN2B subunit ratio of the striatal synaptic NMDAR is a key element in the regulation of motor behaviour and synaptic plasticity in Parkinson's disease (Mellone et al., 2015).

For this reason, it is of great importance to assess whether $\alpha$-syn could alter synaptic transmission and plasticity in striatal SPNs by interfering with different NMDAR subunits.

In the present study, by utilizing a multidisciplinary approach including electrophysiological, optogenetic, immunofluorescence and molecular techniques as well as behavioural testing, we have determined the pathological effects of $\alpha$-syn on the striatal network controlled by NMDAR subunits. 
Finally, we have provided the first proof of concept evidence that antibodies against $\alpha$-syn can prevent these pathological synaptic dysfunctions.

\section{MATERIALS AND METHODS}

All experimental procedures were conducted in conformity with the European Directive 2010/63/EU, in accordance with protocols approved by the Animal Care and Use Committee at the University of Perugia (Perugia, Italy) and IRCCS Fondazione Santa Lucia (Rome, Italy).

\section{Animals}

For electrophysiological and behavioural experiments we used 2-3 month-old male Wistar rats (Charles River, Calco, Italy); 2 month-old transgenic male BAC Drd1a-tdTomato mice containing a mouse Drdla promoter directing the expression of a modified DsRed fluorescent protein (B6.CgTg(Drd1a-tdTomato)6Calak/J, tdTomato, The Jackson Laboratory, Bar Harbor, Maine, U.S.A.) and B6.Cg-Tg(Thy1-COP4/EYFP)18Gfng/J transgenic mice (Thy1-ChR2-YFP) expressing the lightactivated ion channel Channelrhodopsin-2 (ChR2) fused to yellow fluorescent protein under the control of the mouse thymus cell antigen 1 (Thy1) promoter. Selective expression of ChR2 has been demonstrated both in cortical neurons of layer $\mathrm{V}$ and thalamic neurons (Arenkiel et al., 2007; Lim et al., 2012).

Animals were housed at $23^{\circ} \mathrm{C}$ room temperature with food and water ad libitum and a 12-hour light-dark cycle. All efforts were made to minimize the number of animals used and their suffering.

\section{Electrophysiology}

Coronal brain slices including the cortex and the striatum were cut from Wistar rats and tdTomato mice using a vibratome at $220-240 \mu \mathrm{m}$ thickness. To better preserve the connectivity of thalamic fibres to the striatum, para-horizontal slices $(250 \mu \mathrm{m})$ were cut from Thy1-ChR2-YFP mice for optogenetic experiments (Smeal et al., 2007; Ding et al., 2008; Sciamanna et al., 2012). Slices were maintained in a Kreb's solution, bubbled with a $95 \% \mathrm{O}_{2}-5 \% \mathrm{CO}_{2}$ gas mixture at room temperature 
containing (in $\mathrm{mM}$ ): $126 \mathrm{NaCl}, 2.5 \mathrm{KCl}, 1.2 \mathrm{MgCl}_{2}, 1.2 \mathrm{NaH}_{2} \mathrm{PO}_{4}, 2.4 \mathrm{CaCl}_{2}, 10$ glucose, and 25 $\mathrm{NaHCO}_{3}$ (Calabresi et al., 1992b). Single slices were transferred to a recording chamber and submerged in a continuously flowing Krebs' $\left(34{ }^{\circ} \mathrm{C} ; 2.5-3 \mathrm{ml} / \mathrm{minute}\right)$ bubbled with a $95 \% \mathrm{O}_{2}-5 \%$ $\mathrm{CO}_{2}$ gas mixture. The internal solution for path-clamp recordings contained (in $\mathrm{mM}$ ): $145 \mathrm{~K}^{+}-$ gluconate, $0.1 \mathrm{CaCl}_{2}, 2 \mathrm{MgCl}_{2}, 0.1$ EGTA, $10 \mathrm{HEPES}, 0.3 \mathrm{Na}-\mathrm{GTP}$ and $2 \mathrm{Mg}$-ATP, adjusted to $\mathrm{pH}$ 7.3 with $\mathrm{KOH}$. For recording NMDAR-mediated currents, neurons were held at $+40 \mathrm{mV}$ membrane holding potential and the internal solution contained (in $\mathrm{mM}$ ): $120 \mathrm{CsMeSO}_{3}, 10 \mathrm{CsCl}, 8 \mathrm{NaCl}, 2$ $\mathrm{MgCl}_{2}, 10$ HEPES, 0.2 EGTA, 10 TEA, 5 QX314, 0.3 Na-GTP and $2 \mathrm{Mg}$-ATP, adjusted to pH 7.3 with $\mathrm{CsOH}$. Only neurons identified as striatal SPNs were considered for the experiments. Normally, for each animal we obtained 2-3 experiments. SPNs were identified by their hyperpolarized resting membrane potential (RMP, $\sim-80 \mathrm{mV}$ ), absence of spontaneous action potential discharge and presence of tonic firing activity during current-induced membrane depolarization. Signals were amplified with a Multiclamp 700B amplifier (Molecular Devices), recorded and stored on PC using pClamp10. Whole-cell voltage-clamp recordings (access resistance 15-30 M $\Omega$; holding potential $-80 \mathrm{mV}$ ) were performed with borosilicate pipettes (4-7 M $\Omega$ ) filled with the standard internal solution. A bipolar electrode, connected to a stimulation unit (Grass Telefactor), was located in the white matter between the cortex and the striatum to stimulate glutamatergic fibres $(0.1 \mathrm{~Hz})$ and evoke excitatory post-synaptic currents (EPSCs), while the recording electrode was placed in the dorsolateral striatum. In all patch-clamp experiments $50 \mu \mathrm{M}$ picrotoxin was added to the external medium to block $\mathrm{GABA}_{\mathrm{A}}$ receptors. To induce LTP or longterm depression (LTD), a high-frequency stimulation (HFS) protocol consisting of three trains of 3 seconds (20 seconds interval) was delivered at $100 \mathrm{~Hz}$. During HFS protocol, the stimulus intensity was increased to supra-threshold levels. External $\mathrm{Mg}^{2+}$ ions were omitted to maximize the contribution of NMDARs during LTP experiments (Calabresi et al., 1992b). The AMPA receptor (AMPAR)- and NMDAR-mediated synaptic currents (AMPA-EPSCs and NMDA-EPSCs) were 
recorded in pharmacological isolation, with $50 \mu \mathrm{M}$ picrotoxin plus $50 \mu \mathrm{M}$ APV and $50 \mu \mathrm{M}$ picrotoxin plus $10 \mu \mathrm{M} C N Q X$, respectively. AMPAR rectification index (RI) was obtained by dividing the AMPA-EPSC amplitude measured at $+40 \mathrm{mV}$ by the one measured at $-80 \mathrm{mV}$. NMDA/AMPA ratios were calculated by dividing the NMDA-EPSC positive peak amplitude, acquired at $+40 \mathrm{mM}$ holding potential in the presence of $50 \mu \mathrm{M}$ picrotoxin plus $10 \mu \mathrm{M}$ CNQX, by the EPSC negative peak amplitude acquired at $-80 \mathrm{mV}$ in picrotoxin.

\section{Optogenetic}

Optical stimulation of cortex or thalamus was performed using an infinity cube (CAIRN Research, UK), consisting of a $470 \mathrm{~nm}$ filter system mounted on an Olympus BX-51WI microscope and controlled by an Optoflash (CAIRN Research, UK) LED light Switch. The experiments were performed on Thy1-ChR2-YFP mice. Light power at microscope objective exit was 2-30 $\mathrm{mW} / \mathrm{mm}^{2}$. Spotlight was made by utilizing a diaphragm placed along the light path. Slices were illuminated from the top and a round spot was created with a radius of approximately 500-600 $\mu \mathrm{m}$ in order to avoid light spread to unwanted brain structures and delivered on the cortex or the thalamus. A 3 mseconds light pulse was delivered every 15 seconds on cortical layer $\mathrm{V}$ or in the thalamus in order to evoke EPSCs in MSNs.

\section{Preparation of alpha-synuclein in Krebs solution}

Different concentrations of $\alpha$-syn cocktail (3, 10, 30 and $300 \mathrm{nM}$ ) (referred in the text as $\alpha$-syn OLIGO) were prepared in Krebs solution bubbled with a 95\% $\mathrm{O}_{2}-5 \% \mathrm{CO}_{2}$ gas mixture at room. The solutions were incubated up to 1 hour taking aliquots at different time points.

\section{Alpha-synuclein preformed fibrils (PFF) preparation}

Lyophilized fresh monomeric $\alpha$-syn $(0.33 \mathrm{mg}$ ) (recombinant human $\alpha$-syn) was dissolved in sterile PBS to a final concentration of $1 \mathrm{mg} / \mathrm{mL}(\approx 70 \mu \mathrm{M}) .300 \mu \mathrm{L} \alpha$-syn-containing buffer has been incubated for 7 consecutive days at $37{ }^{\circ} \mathrm{C}$ on a shaker $(300 \mathrm{rpm})$ in a sterile 96 -well plate. At the 
end of this procedure the $\alpha$-syn PFF aliquots were stored at $-80{ }^{\circ} \mathrm{C}$ until the use for intra-brain injection.

\section{Transmission electron microscopy (TEM)}

The samples $(5 \mu \mathrm{l})$ were deposited on a glow-discharged Formvar-Carbon 300-mesh coated copper grid (Agar Scientific, UK) for 2 minutes, blotted with Whatman filter paper and negatively stained with $2 \%$ uranyl acetate for few seconds. Grids were examined with an Energy Filtering transmission electron microscope Leo 912 ab (Zeiss, Germany) operating at 100KV and digital images were acquired by a CCD camera (Proscan, Germany) $1 \mathrm{kx} 1 \mathrm{k}$ with the iTEM software (Olympus, Germany).

\section{Detection of alpha-synuclein oligomers in the Krebs solution}

A 384-well ELISA microplate (Nunc MaxiSorb, Nunc) was coated by overnight incubation at $4{ }^{\circ} \mathrm{C}$ with $\mathrm{SynO} 2(0.2 \mu \mathrm{g} / \mathrm{ml})$ in $200 \mathrm{mM} \mathrm{NaHCO} 3$, pH $9.6(50 \mu \mathrm{l} /$ well). The plate was then washed with PBST and incubated with $100 \mu \mathrm{l} /$ well of blocking buffer for 2-hours at $37{ }^{\circ} \mathrm{C}$. After washing, $50 \mu \mathrm{l}$ of the samples $(1 \mathrm{nM})$ was added to each well, and plates were incubated at $37{ }^{\circ} \mathrm{C}$ for 2.5 -hours. FL-140 (rabbit polyclonal antibody, Santa Cruz Biotechnology, Santa Cruz, CA, USA), diluted in blocking buffer at 1:1 K, was added to the appropriate wells, and incubated at $37^{\circ} \mathrm{C}$ for $2 \mathrm{~h}$. Next, the plate was washed and incubated for $2 \mathrm{~h}$ at $37{ }^{\circ} \mathrm{C}$ with $50 \mu \mathrm{l} /$ well of goat anti-rabbit IgG HRP (Jackson ImmunoResearch) diluted in blocking buffer $(1: 15 \mathrm{~K})$. After washing, the plate was incubated with $50 \mu \mathrm{l} /$ well of an enhanced chemiluminescent substrate (SuperSignal ELISA Femto, Pierce Biotechnology, Rockford, IL). The chemiluminescence, expressed in relative light units, was immediately measured using VICTOR ${ }^{\mathrm{TM}}$ X3 multilabel plate reader (PerkinElmer).

\section{Thioflavin $\mathbf{T}$ binding assay}

The formation of $\alpha$-syn oligomers was confirmed by Thioflavin T (ThT) binding assay (Nilsson, 2004). ThT was incubated at a final concentration of $20 \mu \mathrm{M}$ in different $\alpha$-syn solutions (30 $\mathrm{nM}$ and $300 \mathrm{nM}$, respectively) collected at T0 and after 60 minutes of incubation in Krebs solution bubbled 
with a $95 \% \mathrm{O}_{2}-5 \% \mathrm{CO}_{2}$ gas mixture at room temperature. Emission wavelength scan was performed with an excitation wavelength of $450 \mathrm{~nm}$ using a plate reader (BMG Labtech Clariostar). The assay was performed in triplicate.

\section{Immunoprecipitation of alpha-synuclein oligomers formed in Krebs solution}

$500 \mu \mathrm{l}$ of the samples collected at time points 0 and 30 minutes were incubated with 0.043 and 4.3 $\mathrm{X} 10^{-5} \mu \mathrm{g} / \mathrm{ml}$ of either SynO2 or Syn211 antibodies for 2-hours at room temperature. $100 \mu 1$ of protein $\mathrm{G}$ beads was added and incubated for 1-hour at room temperature. The samples were centrifuged and the supernatant were collected and checked for the presence of $\alpha$-syn oligomers by ELISA. The bound $\alpha$-syn oligomers form the beads were eluted by adding $50 \mu 1$ of $0.2 \mathrm{M}$ glycine pH 2.5 and immediately neutralized with $5 \mu$ of 1 M Tris pH 8.0 and taken for western blot analysis.

\section{Subcellular fractionation}

For the extraction of triton-insoluble postsynaptic fraction (TIF), striatal areas were carefully isolated and collected on dry ice from control slices and slices treated with $\alpha$-syn alone (30 nM) and with Abs against $\alpha$-syn (Syn211 and SynO2). Striatal areas were then homogenized with a Teflonglass potter in ice-cold buffer containing (in mM) 320 sucrose, 1 Hepes, $1 \mathrm{MgCl}_{2}, 1 \mathrm{NaHCO}_{3}, 0.1$ phenylmethylsulfonylfluoride at $\mathrm{pH} 7.4$ in the presence of Complete ${ }^{\mathrm{TM}}$ Protease Inhibitor Cocktail Tablets (Roche Diagnostics, Basel, Switzerland) and phosSTOPTM Phosphatase Inhibitor (Roche Diagnostics). The sample was spun at $13.000 \mathrm{~g} \mathrm{x} 15$ minutes at $4{ }^{\circ} \mathrm{C}$. The resulting pellet was resuspended in Triton- $\mathrm{KCl}$ buffer $(0.5 \%$ Triton $\mathrm{X}-100$ and $150 \mathrm{mM} \mathrm{KCl})$ and, after 15 minutes incubation on ice, it was spun further at $100.000 \mathrm{~g} \mathrm{x} \mathrm{1-hour} \mathrm{at} 4{ }^{\circ} \mathrm{C}$. The pellet (triton-insoluble postsynaptic fraction, TIF) was resuspended in $20 \mathrm{mM}$ Hepes buffer supplemented with Complete ${ }^{\mathrm{TM}}$ Protease Inhibitor Cocktail Tablets and stored at $-80{ }^{\circ} \mathrm{C}$.

\section{Western blotting (WB)}


The levels of NMDARs and AMPARs in the TIF were analysed by WB. TIF samples were separated onto a 7\% acrylamide/bis acrylamide gel. Proteins were blotted onto a nitrocellulose membrane (Bio-Rad, Hercules, CA, USA) and probed with the appropriate primary antibodies followed by the corresponding HRP-conjugated secondary antibodies. Labeling detection was performed with ChemiDoc MP Imaging System (Bio-Rad) and images were acquired with ImageLab software (Bio-Rad). The primary antibodies used in this study are anti-GluN2A 1:500 (Sigma-Aldrich, St. Louis, MO, USA), anti-GluN2B 1:500 (Neuromab, Davis, CA, USA), antiGluA1 1:1000 (Neuromab), anti-GluA2 1:500 (Neuromab) and anti-tubulin 1:20000 (SigmaAldrich). The latter was always used as loading control for normalization.

\section{Immunolabeling}

Primary antibodies of hu- $\alpha$-Syn $(1: 1000$, sc-12767, Santa Cruz Biotechnology) in combination with hu- $\alpha$-Syn phosphorylated on Ser129 (hu- $\alpha$-Syn-phospho S129, ab51253; abcam) or in combination with tyrosine hydroxylase (TH 1:500, AB152; Merck Millipore) were used. $30 \mu \mathrm{m}$ coronal slices, obtained with the use of a vibratome (Leica VT1000 S) were incubated overnight at $4{ }^{\circ} \mathrm{C}$ with a PBS solution containing BSA $0.1 \%$ and the combination of two primary antibodies. After rinsing in PBS, the sections were incubated for 2-hours with a mixture of secondary antibodies: goat antirabbit Alexa Fluor 647, goat anti-mouse Alexa Fluor 488 (1:300; AP187SA6 and AP124JA, Merck Millipore). After subsequent washes in PBS, brain slices were incubated with DAPI (S7113; Merck Millipore) for 10 minutes and finally mounted on gelatin-coated slides and coverslipped. The specificity of immunoreaction was controlled by omitting primary antibodies. Images of the medial and lateral striatum (60x magnification) were taken using a fully motorized confocal microscope Nikon A1 and Nikon NIS Elements Advanced Research software.

\section{Surgery}

A total of 30 male Wistar rats (Charles River, Italy) were housed three $\mathrm{x}$ cage with free access to chow and water under a 12:12 h light-dark cycle. All surgical operations were performed under 
ketamine/xylazine anaesthesia. Animals received two bilateral intrastriatal injections of either $\alpha$-syn PFF $(n=9)$, OLIGO $(n=6)$ or PBS (control, $n=15)$. The volume injected using a $10 \mu$ l Hamilton microsyringe was $1 \mu \mathrm{l}$ for each site. The injection rate was $0.38 \mu 1 /$ minute and for each site the cannula was left in place for additional $2 \mathrm{~min}$ before retracting it. The coordinates, based on a previous study using these parameters to perform striatal lesions (Kirik et al., 1998), were the following: 1) anteroposterior (AP) +1.0; mediolateral (ML) +3.0; dorsoventral (DV) -5.0; 2) AP +1.0; $\mathrm{ML}$-3.0; DV -5.0. ML coordinates were taken from lambda, AP coordinates were from bregma, according to Paxinos and Watson (Paxinos, 2005). Tooth bar was set at 0.00. After the surgery the animals were monitored until awake and then brought back to animal facility. Six weeks after the surgery the animals were tested for motor behaviour and cognition deficit.

\section{Visuo-Spatial Memory Task}

Visuo-Spatial Memory Task was a rat adapted version of the visuo-spatial memory task previously described in mouse (De Leonibus et al., 2007; De Leonibus et al., 2009; Rodo et al., 2017). The test was divided in 10 sessions of 5 minute each. The interval between the sessions was 3 minutes. Briefly, during session 1 (S1), rats were placed in an empty a square open field to habituate to the context $(60 \times 60 \mathrm{~cm})$. After this, from session 2 to session $7(\mathrm{~S} 2-\mathrm{S} 7)$, rats were free to explore three objects positioned in the arena to allow them to learn information about the objects and their spatial location in the arena (habituation phase). To test whether the animals learned spatial information related to the position of the objects, during the spatial test session 8 (S8), the objects configuration was changed by moving one object (the displaced object, DO) and leaving the other two objects in the same position (non-displaced objects, NDO1 and NDO2). In session 9 (S9), the configuration of the objects was kept unchanged to let the animal to habituate to the spatial change. In the last session (S10), to test whether animals learned information about the shape of the object, one of the familiar objects was replaced by a new one (substituted object, SO); the other two objects were left unchanged (non-substituted objects, NSO1 and NSO2). Object exploration was defined as the time 
in which the nose of the animal was in directed toward the object ( $<2 \mathrm{~cm}$ distance). The animals' ability to selectively react to the spatial change was analysed by calculating the spatial exploration index $(\mathrm{DO}=\mathrm{DO}[\mathrm{S} 8]-\mathrm{DO}[\mathrm{S} 7] ; \mathrm{NDO}=\mathrm{NDO}[\mathrm{S} 8]-\mathrm{NDO}[\mathrm{S} 7])$, while the animals' ability to selectively react to the non-spatial change (novel object) was analysed by calculating non-spatial exploration index $(\mathrm{SO}=\mathrm{SO}[\mathrm{S} 10]-\mathrm{SO}[\mathrm{S9}] ; \mathrm{NSO}=\mathrm{NSO}[\mathrm{S} 10]-\mathrm{NSO}[\mathrm{S} 9])$. Basal motor activity was evaluated during S1, by measuring time interval changes in distance travelled $(\mathrm{cm})$, immobility time (seconds) and maximal speed (cm/second).

\section{Statistical Analysis}

Data analysis of electrophysiological experiments was performed off-line using Clampfit10 (Molecular Devices) and GraphPad Prism 5.0 (GraphPad Software). Values in the text and figures are mean \pm standard error of the mean (SEM), $n$ representing the number of recorded neurons. Changes of EPSC amplitude induced by drugs or by stimulation protocols were expressed as a percentage of the baseline, the latter representing the normalized EPSC mean amplitude acquired during a stable period (10-15 minutes) before delivering drugs or stimulation and statistically evaluated by the Student's $t$-test for unpaired samples. LTP and LTD presence were statistically verified with the Student's $t$-test for paired samples by comparing in each experiment values of the EPSC amplitudes at 25-30 minutes after the application of the HFS protocol relatively to the baseline. Current-voltage (I-V) curves were compared using the two-way ANOVA. The significance level was established at $\mathrm{P}<0.05$. For WB experiments, significance of the differences was determined by one-way ANOVA followed by Tukey's post-hoc test or by unpaired Student's $t$ test as appropriate. For ThT binding assay significance of the differences was determined by Student's $t$-test. For behavioural analysis, data were analysed by using two-way Analysis of Variance (ANOVA) for repeated measure, with treatment (two levels: control and $\alpha$-syn) as between factor. Duncan post hoc analysis was used when appropriate. 
When parametric tests were used, normality was checked with Shapiro-Wilk test and visual inspection of data distribution. The criterion for significance was set at $\mathrm{P}<0.05$. We used ShapiroWilk test for its level of power compared to other procedures in small sample size scenarios (Royston, 1993).

\section{Drugs}

Picrotoxin, D-(-)2-amino-5-phosphonopentanoic acid (APV), 6-cyano-7- nitroquinoxaline-2,3dione disodium (CNQX), threo ifenprodil hemitartrate, 3-chloro-4-fluoro-N-[4-[[2(phenylcarbonyl)hydrazino]carbonyl]benzyl]benzenesulfonamide (TCN-201), NVP-AAM077 were from Tocris; dopamine hydrochloride (DA) was from Sigma.

Drugs were bath-applied by switching the Kreb's solution to one containing known concentrations of drugs. Drugs applied in the recording chamber were delivered for at least 10 minutes before recording AMPA-EPSC and NMDA-EPSC and maintained throughout the experiment. We used two different mouse antibodies against $\alpha$-syn: a monoclonal IgG1 Ab, raised against the amino acid 121-125 of human $\alpha$-syn, which recognizes all forms of the protein (Syn211, Santa Cruz Biotechnology, Inc.,) (El-Agnaf et al., 2003) and a monoclonal IgG1 Ab which has a higher affinity to oligomers more than $\alpha$-syn fibrils (SynO2) (Vaikath et al., 2015). Abs and $\alpha$-syn were provided by Prof. O. El-Agnaf (U.A.E. University, United Arab Emirates). Corticostriatal slices were incubated in a solution containing $8.6 \mu \mathrm{g} \alpha$-syn, corresponding to a dose of $30 \mathrm{nM}$, plus the $\mathrm{Ab}$ for 1 hour $(1: 1, \mu \mathrm{g} / \mu \mathrm{g})$. The Syn211 Ab was further diluted 1:10 $(0.043 \mu \mathrm{g} / \mathrm{ml})$ and 1:100 $(0.0043$ $\mu \mathrm{g} / \mathrm{ml})$, while SynO2 Ab was used at dilution 1:10.000 $\left(4.3 \times 10^{-5} \mu \mathrm{g} / \mathrm{ml}\right)$.

\section{RESULTS}

Alpha-synuclein does not affect basal membrane properties and LTD but impairs LTP in striatal SPNs 
In order to characterize the $\alpha$-syn effect on striatal SPNs we first explored the effect of this protein on the basal membrane properties performing patch-clamp recordings of SPNs in striatal slices obtained from 2-3 months-old rats. The current-voltage relationship was evaluated applying hyperpolarizing and depolarizing steps of currents to SPNs of control slices and of slices incubated for 1-hour with $30 \mathrm{nM}$ monomeric/oligomeric $\alpha$-syn freshly diluted in the Kreb's solution (see Materials and Methods). We found that the measured electrical properties were unaffected by $\alpha$-syn since statistical analysis showed no significant differences in the I-V curves between SPNs recorded in control slices $(\mathrm{n}=5)$ and in slices treated with $\alpha$-syn $(\mathrm{n}=4)$ (control $v s \alpha$-syn, $\mathrm{P}>0.05)$ (Fig. 1A). Striatal slices were subsequently tested to assess the effect of $30 \mathrm{nM} \alpha$-syn on long-term changes of synaptic transmission (LTD and LTP) of SPNs. EPSCs were recorded for 10 minutes to obtain a stable baseline and then for 30 minutes after the application of the HFS protocol. We found that SPNs recorded in control and in $\alpha$-syn-incubated slices showed LTD of similar amplitudes (Calabresi et al., 1992a). In fact, the EPSCs amplitude was reduced by $46.0 \pm 2.8 \%(\mathrm{n}=6)$ and by $49.8 \pm 2.1 \%(\mathrm{n}=6)$ in SPNs from control slices and $\alpha$-syn-incubated slices, respectively $\left(\mathrm{t}_{(10)}=1.094\right.$, $\mathrm{P}<0.05$, pre vs 30min after HFS, Fig. 1B). To explore the effect of $\alpha$-syn on SPNs LTP, EPSCs were recorded before and for 30 minutes after the application of magnesium free HFS protocol. While SPNs from control slices showed a robust LTP of $148.7 \pm 3.2 \%\left(\mathrm{n}=7, \mathrm{t}_{(6)}=15.37, \mathrm{P}<0.001\right)$, neurons from $\alpha$-syn-treated slices showed no LTP $(n=7, P>0.05$, pre $v s 30 \mathrm{~min}$ after HFS, Fig. 1C). We also analysed the EPSC amplitude, measured $30 \mathrm{~min}$ after the induction of the LTP, in the presence of different concentrations of $\alpha$-syn. We found that while $3 \mathrm{nM} \alpha$-syn (n=6) did not affect this parameter, both $10 \mathrm{nM}(\mathrm{n}=7)$ and $30 \mathrm{nM}(\mathrm{n}=7)$ produced a significant reduction. Higher concentrations of $\alpha$-syn (300 nM, n=6) did not further decrease the EPSC amplitude (Fig. 1D). It has been postulated a major detrimental effect of $\alpha$-syn is the reduction of striatal dopamine (DA) levels (Ghiglieri et al., 2018), thus the $\alpha$-syn-induced LTP impairment could be induced by lower DA levels in the synaptic cleft. To test this hypothesis, $30 \mu \mathrm{M}$ exogenous DA were bath applied 
before the HFS in order to obtain a recovery of the LTP in the presence of $\alpha$-syn $(n=6)$. Surprisingly, DA failed to restore this form of synaptic plasticity suggesting that other nondopaminergic mechanisms might be involved in the loss of LTP in the presence of $\alpha$-syn (Fig. 1E). In order to investigate the time- and dose-dependent formation of $\alpha$-syn oligomers in the Krebs solution used for the electrophysiological experiments, $\alpha$-syn samples at time 0 and 30 min were taken and measured by specific oligomeric ELISA. As expected, the formation of $\alpha$-syn oligomers was time- and dose-dependent (Fig. $1 \mathrm{~F},{ }^{*} \mathrm{P}<0.05,{ }^{*} * \mathrm{P}<0.01$ ), as higher level of $\alpha$-syn oligomers were detected in the $300 \mathrm{nM} \alpha$-syn samples collected at $30 \mathrm{~min}$. To evaluate the presence of fibrils we used the Thioflavin T (ThT) fluorescence assay; the results clearly show that little fluorescence was detected with the $30 \mathrm{nM}$ concentration, independently on the timing of sampling (Fig. 1G, $* * * \mathrm{P}<0.001)$

\section{Alpha-synuclein affects SPNs glutamatergic transmission by selectively targeting the GluN2A- NMDAR current, but not the GluN2B-NMDAR and AMPAR currents}

We then evaluated the effect of $\alpha$-syn on the AMPA- and NMDA-EPSCs since both these glutamate receptors exert a pivotal role in the long-term changes of synaptic plasticity of SPNs. To isolate AMPA-EPSCs, SPNs were recorded in the presence of $50 \mu \mathrm{M}$ of the NMDAR antagonist APV and $50 \mu \mathrm{M}$ of the $\mathrm{GABA}_{\mathrm{A}} \mathrm{R}$ antagonist picrotoxin. After obtaining stable EPSCs for 10 minutes, synaptic currents were measured in the presence of $30 \mathrm{nM} \alpha$-syn for additional 10 minutes. We found no significant difference between AMPA-EPSCs recorded before and following $\alpha$-syn application $(\mathrm{n}=6, \mathrm{P}>0.05$, Fig. $2 \mathrm{~A})$. We also evaluated the AMPAR rectification index (RI) calculated in control conditions and in the presence of $30 \mathrm{nM} \alpha$-syn (Fig. 2B). Statistical analysis shows that in these conditions the AMPA RI was not significantly different from that measured before $\alpha$-syn application ( $n=6, P>0.05$, Fig. $2 B$ ), further suggesting that in SPNs $\alpha$-syn does not alter the function of AMPAR. 
We also explored whether $\alpha$-syn affected the NMDAR by targeting the GluN2A- or the GluN2Bmediated NMDA-EPSC (GluN2A-EPSC and GluN2B-EPSC). To isolate the GluN2A-EPSC, synaptic responses were recorded in the presence of $10 \mu \mathrm{M}$ of the AMPAR antagonist CNQX, 50 $\mu \mathrm{M}$ picrotoxin and $3 \mu \mathrm{M}$ of the GluN2B antagonist ifenprodil. We found that after recording a stable baseline for 10 minutes, the subsequent application of $30 \mathrm{nM} \alpha$-syn significantly reduced the GluN2A-EPSC by $40.8 \pm 6.1 \%\left(n=6, \mathrm{t}_{(5)}=6.684, \mathrm{P}<0.05\right.$, Fig. $\left.2 \mathrm{C}\right)$. We subsequently isolated the GluN2B-EPSC by recording synaptic responses in the presence of CNQX, $50 \mathrm{nM}$ of the GluN2A antagonist NVP-AAM077 and picrotoxin. The application of $30 \mathrm{nM} \alpha$-syn did not affect the GluN2B-EPSC ( $n=6, P>0.05$, Fig. 2D). Similarly, we found that the GluN2B-EPSC, isolated using the more specific GluN2A antagonist TCN-201 (10 $\mu \mathrm{M})$, was not significantly affected by $\alpha$-syn application ( $n=6, P>0.05$, Fig. 2E). Taken together these findings show that $\alpha$-syn affects the SPNs NMDA-EPSC selectively targeting the GluN2A-containing NMDA receptors. Moreover, we also evaluated, by WB analysis in a triton-insoluble post-synaptic fraction (TIF), the possibility that alterations in the molecular composition of ionotropic glutamate receptors may lead to the observed synaptic alterations in SPNs induced by $\alpha$-syn. In line with the electrophysiological experiments, we found that incubation (1-hour) of the slices in $30 \mathrm{nM} \alpha$-syn did not alter GluA1 and GluA2 AMPAR subunit synaptic levels ( $\mathrm{n}=8, \mathrm{P}>0.05, \alpha$-syn $v$ s control, Fig. $2 \mathrm{~F}$ ) while it significantly reduced the GluN2A but not GluN2B NMDAR subunit synaptic levels ( $\mathrm{n}=6$, GluN2A, $\mathrm{P}=0.014$, $\alpha$-syn vs control, Fig. 2G).

\section{GluN2A but not GluN2B NMDAR subunit plays a critical role in the induction of LTP in SPNs}

To ascertain the involvement of GluN2A and GluN2B NMDAR subunits in LTP, we first induced this form of synaptic plasticity in the presence of $3 \mu \mathrm{M}$ of the GluN2B antagonist ifenprodil, to isolate the GluN2A-EPSC. In these conditions we found a normal LTP since the increase of EPSCs 
amplitude obtained after the delivery of HFS protocol was not different from that one of control slices (control, $147.2 \pm 3.0 \%, \mathrm{n}=6$; ifenprodil, $143.3 \pm 2.8 \%, \mathrm{n}=5, \mathrm{P}>0.05$, Supplementary Fig. 1). Conversely, the application of the GluN2A antagonists NVP-AAM077 (50 nM, n=5) or TCN-201 (10 $\mu \mathrm{M}, \mathrm{n}=5)$ completely prevented the LTP induction $(\mathrm{P}>0.05$, Supplementary Fig. 1), further confirming that the NMDAR expressing the GluN2A subunit mediates the LTP induction of striatal SPNs.

\section{Alpha-synuclein affects LTP and NMDAR-mediated synaptic currents in SPNs of both direct and indirect pathways}

To investigate whether SPNs expressing either the D1-like or D2-like DA receptors (Calabresi et $a l ., 2014)$ were differentially affected by $\alpha$-syn, we recorded SPNs from striatal slices of dtTomato mice. In these animals, we firstly optically identified fluorescent (D1-like expressing: D1Rpositive) and non-fluorescent (putative D2-like expressing: putative D2R-positive) SPNs (Fig. 3A). Subsequently, we patch-clamped SPNs for an electrophysiological characterization and for LTP analysis in both these neuronal sub-populations. We found that both fluorescent (D1R-positive) and non-fluorescent (putative D2R-positive) SPNs showed LTP of similar amplitude. In fact, 30 minutes after the induction of the HFS protocol, the EPSC amplitude was increased respect to the baseline by $134.6 \pm 4.0 \%\left(\mathrm{n}=6, \mathrm{t}_{(5)}=11.33, \mathrm{P}<0.001\right)$ and $137.1 \pm 5.9 \%\left(\mathrm{n}=6, \mathrm{t}_{(5)}=5.452, \mathrm{P}<0.001\right)$, respectively in fluorescent and non-fluorescent SPNs (Fig. 3B).

Moreover, we aimed at verifying whether $\alpha$-syn equally affected LTP of SPNs of both neuronal sub-populations. Striatal slices from dtTomato mice were incubated for 1-hour with $30 \mathrm{nM} \alpha$-syn. In these conditions we found that the induction of LTP was completely prevented in both fluorescent ( $n=5, P>0.05)$ and non-fluorescent SPNs ( $n=5, P>0.05)$ (Fig. 3C-D).

Since $\alpha$-syn affected NMDA-EPSCs (Fig. 2C-E), we tested whether this glutamatergic current was differentially altered by $\alpha$-syn in SPNs expressing and not expressing (putative D2R-positive) the 
D1 DA receptor. The NMDA-EPSC amplitudes were recorded in pharmacological isolation in the presence of $50 \mu \mathrm{M}$ picrotoxin plus $10 \mu \mathrm{M}$ CNQX in both fluorescent and non-fluorescent SPNs of dtTomato mice. After acquiring a stable baseline for 10 minutes, $30 \mathrm{nM} \alpha$-syn was bath applied for subsequent 10 minutes. We found that $\alpha$-syn significantly reduced the NMDA-EPSC in both SPN populations. The EPSCs were reduced by $27.2 \pm 4.2 \%$ in D1R-positive SPNs $\left(n=6, t_{(5)}=6.510\right.$, $\mathrm{P}<0.05$, Fig. 3E) and by $30.1 \pm 6.3 \%$ in putative D2R-positive SPNs $\left(\mathrm{n}=4, \mathrm{t}_{(3)}=4.764, \mathrm{P}<0.05\right.$, Fig. $3 \mathrm{~F})$ and the amplitude of these reductions was similar in these two groups of SPNs $(\mathrm{P}>0.05)$. These findings suggest that $\alpha$-syn exerts similar effects in SPNs of the direct and indirect basal ganglia pathways equally affecting the NMDAR-mediated synaptic current.

\section{Alpha-synuclein affects the NMDAR-current evoked in SPNs by cortico-striatal and thalamo- striatal optical stimulation}

Our data suggest that $\alpha$-syn reduced the GluN2A-EPSC amplitude following the stimulation of glutamatergic afferents to the striatum. However, striatal SPNs receive glutamatergic inputs by both the cortex and the thalamus and these pathways might be differentially regulated by pharmacological agents (Wu et al., 2015). Thus, we investigated whether $\alpha$-syn affected the NMDAR-mediated transmission in cortico-striatal synapses as well as in the thalamo-striatal pathway by using an optogenetic approach to better dissect inputs from these two brain structures. We firstly recorded NMDA-EPSCs in pharmacological isolation to evaluate the contribution of the GluN2A and GluN2B NMDAR subunits in cortico-striatal and thalamo-striatal synapses and then analysed the effect of $\alpha$-syn on these currents (Fig. 4A-H). SPNs EPSCs, evoked by optical stimulation of cortico-striatal projections, were recorded from Thy1-ChR2-YFP mice. GluN2A- or GluN2B-EPSCs were isolated in the presence of $50 \mu \mathrm{M}$ picrotoxin, $10 \mu \mathrm{M}$ CNQX and $3 \mu \mathrm{M}$ ifenprodil (GluN2B antagonist) or $10 \mu \mathrm{M}$ TCN-201 (GluN2A antagonist), respectively. 
We found that the NMDA-EPSC was significantly reduced by $45.46 \pm 3.29 \%$ in the presence of the solution containing ifenprodil and by $35.04 \pm 5.47 \%$ in that with TCN-201 (ifenprodil, $\mathrm{n}=6$, $\mathrm{t}_{(5)}=13.86, \mathrm{P}<0.0001 ; \mathrm{TCN}-201, \mathrm{n}=6, \mathrm{t}_{(5)}=6.405, \mathrm{P}<0.05$, Fig. 4C). We subsequently tested the effect of $\alpha$-syn on the isolated GluN2A- and GluN2B-EPSC. $30 \mathrm{nM} \alpha$-syn significantly reduced the GluN2A-EPSC by $40.82 \pm 4.46 \%\left(\mathrm{n}=6, \mathrm{t}_{(5)}=9.148, \mathrm{P}<0.05\right.$, Fig. 4D). This effect was observed at 5 , but not at 2 minutes, after the $\alpha$-syn application (at least $n=4$ for all the time points, $\mathrm{P}<0.001$, Fig. 4D). Notably, longer $\alpha$-syn applications (10 minutes) did not further decrease the NMDA current. Interestingly, we found no effect of $\alpha$-syn on the GluN2B-EPSC at all time-points tested (at least $\mathrm{n}=4$ for all time points, $\mathrm{P}>0.05$, Fig. 4D).

Striatal SPNs EPSCs were also evoked by optical stimulation of the thalamus of Thy1-ChR2-YFP mice to evaluate the contribution of the GluN2A and GluN2B currents in the NMDAR response activated by thalamic glutamatergic fibres. NMDA-EPSCs, recorded in the presence of picrotoxin plus CNQX, were significantly reduced by $34.08 \pm 2.87 \%$ in the presence of ifenprodil $(\mathrm{n}=5$, $\mathrm{t}_{(4)}=11.87, \mathrm{P}<0.05$, Fig. $\left.4 \mathrm{~F}\right)$ and by $32.98 \pm 3.12 \%$ in TCN-201 $\left(\mathrm{n}=6, \mathrm{t}_{(5)}=10.57, \mathrm{P}<0.05\right.$, Fig. 4F $)$. Isolated GluN2A and GluN2B currents were subsequently measured in the presence of $\alpha$-syn. We found that in these conditions the GluN2A-EPSCs were reduced by $42.35 \pm 4.0 \%\left(\mathrm{n}=7, \mathrm{t}_{(6)}=10.58\right.$, $\mathrm{P}<0.0001$, Fig. 4G) while, similarly to what observed for GluN2B-EPSCs evoked by optical cortico-striatal stimulation, $\alpha$-syn had no effect on the GluN2B currents ( $n=7, P>0.05$, Fig. 4G). This effect was observed at 5 after the $\alpha$-syn application. Notably, also in this case longer $\alpha$-syn applications did not further decrease the NMDA current and we found no effect of $\alpha$-syn on the GluN2B-EPSC at all time-points tested (at least $\mathrm{n}=3$ for all time points, $\mathrm{P}>0.05$, Fig. 4G).

\section{Monoclonal antibodies targeting $\alpha$-synuclein rescue the impairment of LTP}

Alpha-synuclein is a small protein with intrinsic tendency to aggregate. Oligomeric forms of the protein can impair neuronal function (Diogenes et al., 2012; Rockenstein et al., 2014). Accordingly, 
we found that $30 \mathrm{nM} \alpha$-syn cause LTP loss in SPNs by affecting the GluN2A NMDAR-mediated current. To counteract the effect of $\alpha$-syn on SPN LTP we used the monoclonal Ab Syn211, that recognizes all forms of human $\alpha$-syn (Giasson et al., 2000) and the $\mathrm{Ab} \mathrm{SynO} 2$, which mainly recognizes early soluble oligomers and late aggregates amyloid fibrils of $\alpha$-syn (Majbour et al., 2016).

Incubation of the slices for 1 hour with the Syn211 Ab 1:10 alone did not affect LTP $(158.3 \pm 7.4 \%$, $\mathrm{n}=7, \mathrm{t}_{(6)}=6.863, \mathrm{P}<0.001$, Fig. $\left.5 \mathrm{~A}\right)$. However, incubation of the slices for 1 hour with the Syn211 $\mathrm{Ab}$ 1:10 in combination with $30 \mathrm{nM} \alpha$-syn was able to prevent the loss of LTP induced by $\alpha$-syn (153.0 $\pm 54.9 \%, \mathrm{n}=7, \mathrm{t}_{(6)}=10.52, \mathrm{P}<0.0001$, Fig. 5A). Conversely, a lower Ab concentration (1:100) was not sufficient to counteract the $\alpha$-syn effect on LTP amplitude; in fact, SPNs recorded in the presence of Syn211 Ab 1:100 plus 30 nM $\alpha$-syn showed no LTP (n=7, P>0.05, Supplementary Fig. 2).

We subsequently tested the effect of SynO2 $\mathrm{Ab}$, which recognizes oligomeric forms of $\alpha$-syn more specifically than fibrillar forms. We found that while a very low concentration $(1: 10.000)$ of this $\mathrm{Ab}$ did not affect LTP per se $\left(143.2 \pm 3.8 \%, \mathrm{n}=7, \mathrm{t}_{(6)}=13.33, \mathrm{P}<0.0001\right.$, Fig. $\left.5 \mathrm{~B}\right)$, it rescued this form of synaptic plasticity when incubated in the presence of $30 \mathrm{nM} \alpha$-syn $\left(140.9 \pm 3.8 \%, \mathrm{n}=7, \mathrm{t}_{(6)}=12.01\right.$, $\mathrm{P}<0.0001$, Fig. 5B). Taken together these data suggest that Abs against $\alpha$-syn represent a valid tool for preventing the detrimental effect of $\alpha$-syn on striatal synaptic plasticity.

Next, we tested the ability of SynO2 and Syn211 antibodies to capture $\alpha$-syn oligomers formed during incubation in Krebs solution. We conducted immunoprecipitation experiment with the samples collected at time 0 and 30 minutes with antibodies (SynO2 or Syn211) and estimated the $\alpha$ syn oligomers left in the sample using our oligomeric ELISA. Interestingly, when the samples were immunoprecipitated using SynO2, the supernatant contained very low levels of $\alpha$-syn oligomers with both high and low concentrations (Fig. 5C, left and right panel respectively), suggesting sequestration of $\alpha$-syn oligomers species with high efficacy (Fig. 5C, $* * \mathrm{P}<0.01$ ). Whereas, 
incubation with Syn211 antibody exerted a milder effect, and higher levels of $\alpha$-syn oligomers were detected in the supernatant (Fig. $5 \mathrm{C}, * * \mathrm{P}<0.01$ ). This dose-response nicely parallels that observed in their relative efficacy in rescuing the LTP impairment induced by $\alpha$-syn oligomers (Fig. 5A-B).

\section{Incubation with alpha-synuclein specifically affects GluN2A localization at the post-synaptic} membrane

Notably, incubation of the slices with Syn211 Ab 1:10 or with SynO2 Ab 1:10.000 in combination with $30 \mathrm{nM} \alpha$-syn was sufficient to prevent the reduction of GluN2A levels in the postsynaptic compartment ( $\mathrm{n}=6-11, \mathrm{P}<0.01$, control vs $\alpha$-syn; $\mathrm{P}<0.05$, Syn211 plus $\alpha$-syn $v$ s $\alpha$-syn; $\mathrm{P}<0.05$, SynO2 plus $\alpha$-syn vs $\alpha$-syn, Fig. 5D-E). In agreement with LTP results (see Supplementary Fig. 2), incubation of the slices with Syn211 Ab 1:100 did not revert the effect of $30 \mathrm{nM} \alpha$-syn on GluN2A synaptic levels ( $\mathrm{n}=7-11, \mathrm{P}<0.01$, control $v s \alpha$-syn, Supplementary Fig. 3). No effect of $30 \mathrm{nM} \alpha$-syn on synaptic localization of GluN2B subunit of NMDAR or GluA1 and GluA2 subunits of AMPAR was observed either in absence or presence of co-incubation with Syn211 Ab 1:10 or with SynO2 Ab 1:10.000 (Supplementary Fig. 4A-D). Overall these results indicate that the observed decrease in GluN2A-NMDARs current induced by $\alpha$-syn is directly correlated to a reduction of the synaptic availability of this receptor subunit.

\section{Intrastriatal injections of alpha-synuclein protofibrils impairs visuo-spatial learning}

To test in vivo the effects of human $\alpha$-syn aggregates, we injected directly into the dorsal striatum preformed $\alpha$-syn fibrils (PFF), which is an already characterized progressive model of synucleinopathy. In fact, using a similar approach, a time-dependent spreading of $\alpha$-syn and neuronal degeneration has been reported about 9 months after the injection (Luk et al., 2012), while spreading all over the brain. Figure 6A shows the Transmission Electron Microscopy (TEM) analysis of protofibrils and shorter aggregates (putative oligomers) injected into the rat striatum. We 
decided to test the effects at 6 weeks post-injection (Fig. 7A-B); at this time point aggregates, and other $\alpha$-syn forms, are evidenced through their reaction with the phospho-Ser129hu- $\alpha$-Syn antibody (in red) and remained localized into the dorsal striatum (Fig. 6B-C).

To test whether behavioural performance was affected, PBS- and $\alpha$-syn-injected rats were subjected to a visuo-spatial learning task. The task is based on a habituation/dishabituation paradigm in which animals during the habituation phase (from session 2 to session 7), through direct exploration, acquire information about 3 different objects and their spatial location in the arena (Fig. 7A-B). After this acquisition phase, one of the objects is displaced (session 8); the spatial preference for the displaced object (DO) over the non-displaced objects (NDO) is an index of spatial novelty detection. As reported in figure 7B, $\alpha$-syn-injected rats, as well as PBS-injected animals, explore the objects during the habituation phase, reducing the exploration time across sessions [Treatment $\left(\mathrm{F}_{1,15}=0.026 ; \mathrm{P}=0.874\right)$; Exploration $\left(\mathrm{F}_{5,75}=7.673 ; \mathrm{P}<0.0001\right)$; Exploration $\mathrm{x}$ Treatment $\left(\mathrm{F}_{5,75}=0.75\right.$; $\mathrm{P}=0.589$ )] (Fig. 7B). After the spatial displacement the PBS group showed a preference for DO as compared to NDO1 and NDO2, as expected; in contrast, the $\alpha$-syn-injected group did not show any preference for the $\mathrm{DO}$ [Treatment $\left(\mathrm{F}_{1,15}=0.152 ; \mathrm{P}=0.702\right) ; \mathrm{DO}\left(\mathrm{F}_{2,30}=3.387 ; \mathrm{P}=0.047\right) ; \mathrm{DO} \times$ Treatment $\left.\left(\mathrm{F}_{2,30}=3.347 ; \mathrm{P}=0.049\right)\right]$. This effect was not due to a general effect on object exploration, attention or motivation to explore novelty, as both groups showed a clear preference when the same object was substituted with a new one ( $\mathrm{SO})$ during the last testing session [Treatment $\left(\mathrm{F}_{1,15}=0.348\right.$; $\mathrm{P}=0.564) ; \mathrm{SO}\left(\mathrm{F}_{2,30}=15.153 ; \mathrm{P}<0.0001\right)$; $\mathrm{SO} \times$ Treatment $\left.\left(\mathrm{F}_{2,30}=0.87 ; \mathrm{P}=0.429\right)\right]$.

\section{Intrastriatal injections of alpha-synuclein aggregates impair the SPNs GluN2A-bearing NMDAR-current}

We subsequently attempted to test the possible causal link among the presence of striatal $\alpha$-syn aggregates, the synaptic transmission deficits, as observed in the in vitro electrophysiological 
experiments using $\alpha$-syn oligomers, and the behavioural deficits of $\alpha$-syn-injected rats in performing the visuo-spatial learning task.

A group of human $\alpha$-syn- and sham-injected rats were sacrificed one week after the behavioural task. SPNs of the two groups were patch-clamped and EPSCs were evoked at $-80 \mathrm{mV}$ holding potential, in the presence of $50 \mu \mathrm{M}$ picrotoxin, and at $+40 \mathrm{mV}$, in picrotoxin plus $10 \mu \mathrm{M}$ CNQX, to calculate the NMDA/AMPA ratio of the synaptic current. Statistical analysis revealed that SPNs of $\alpha$-syn-injected rats presented reduced NMDA/AMPA ratio in respect to sham-injected animals $(\alpha-$ syn $v$ s sham, $\mathrm{n}=5$ for each group, $\mathrm{t}$-test, $\mathrm{t}_{(8)}=6.6, \mathrm{P}<0.001$; Fig. $7 \mathrm{C}$ ). In addition, since $\alpha$-syn oligomers specifically reduced the GluN2A-bearing NMDAR-currents (Fig. 2C), EPSCs of SPNs from $\alpha$-syn- and sham-injected rats were also recorded at $+40 \mathrm{mV}$ holding current in the presence of CNQX plus $3 \mu \mathrm{M}$ ifenprodil to measure the GluN2A-EPSC amplitude. We found that in SPNs of $\alpha-$ syn-injected rats the GluN2A-current was significantly smaller than that of sham rats ( $\alpha$-syn vs sham in ifenprodil, $\mathrm{t}$-test, $\mathrm{t}_{(11)}=2.2, \mathrm{P}<0.05$; Fig. 7D) confirming that the spreading of $\alpha$-syn aggregates into the striatum affects the striatal synaptic transmission by reducing the SPN GluN2ANMDA current with significant implications on behavioural learning.

\section{Behavioural effects produced by intrastriatal injection of oligomeric alpha-synuclein}

Intrastriatal injections of human $\alpha$-syn aggregates, containing protofibrils but also shorter forms (see Fig. 6A), clearly produced behavioural and synaptic alterations (See Fig. 7). These alterations could be due either to the action of oligomeric and monomeric $\alpha$-syn forms, similar to those applied in our in vitro experiments, or to the critical presence of fibrillary PFF forms of this protein. Thus, to better dissect the pathogenetic role of different species of $\alpha$-syn (protofibrils versus oligomers and monomers), we performed a similar in vivo intrastriatal injection with $\alpha$-syn cocktail mainly containing oligomers (OLIGO). 
We injected the $\alpha$-syn cocktail ( $\alpha$-syn OLIGO) directly into the dorsal striatum (Fig. 8A-B), using the same protocol used for PFF. As previously indicated for PFF (see Fig. 6A), also the presence of $\alpha$-syn OLIGO has been detected by TEM (Fig. 8A). Electron Microscopy was always used to analyse the quality of $\alpha$-syn preparations before injection into the rat striatum confirming the presence of oligomers with very few protofibrils (Fig. 8A). In addition, $\alpha$-syn OLIGO has been confirmed by immunofluorescence with antibodies against $\alpha$-syn and phospho- $\alpha$-syn (Fig. 8B-C).

As reported in Fig. 8D-F, $\alpha$-syn OLIGO-injected rats, as well as PBS-injected animals, explore the objects during the habituation phase, reducing the exploration time across sessions [Treatment $\left(\mathrm{F}_{1,10}=0.86 ; \mathrm{P}=0.375\right)$; Exploration $\left(\mathrm{F}_{5,50}=9.65 ; \mathrm{P}<0.0001\right)$; Exploration $\mathrm{x}$ Treatment $\left(\mathrm{F}_{5,50}=0.45\right.$; $\mathrm{P}=0.80)$ ] (Fig. 8D). The $\alpha$-syn OLIGO-injected group did not show any preference for the DO [Treatment $\left(\mathrm{F}_{1,10}=0.138 ; \mathrm{P}=0.702\right) ; \mathrm{DO}\left(\mathrm{F}_{2,20}=8.039 ; \mathrm{P}=0.0027\right) ; \mathrm{DO} \times$ Treatment $\left(\mathrm{F}_{2,20}=4.7\right.$; $\mathrm{P}=0.02)]$ and $\mathrm{SO}$ [Treatment $\left(\mathrm{F}_{1,10}=0.713 ; \mathrm{P}=0.41\right) ; \mathrm{SO}\left(\mathrm{F}_{2,20}=8.70 ; \mathrm{P}=0.0019\right) ; \mathrm{SO} \times$ Treatment $\left.\left(\mathrm{F}_{2,20}=3.99 ; \mathrm{P}=0.03\right)\right]$ (Fig. 8E-F), thus suggesting that it impaired both spatial and object novelty recognition, in the absence of major degeneration of $\mathrm{TH}$ positive terminals in the striatum (Supplementary Fig. 5).

Analysis of basal motor activity in the open field reveals that at this early stage neither animals injected with protofibrils nor animals injected with $\alpha$-syn OLIGO show significant changes of motor behaviour (Supplementary Fig. 6).

\section{DISCUSSION}

In this study we have investigated the effects of exogenous human $\alpha$-syn on synaptic transmission and plasticity of SPNs, the main neuronal subtype of the striatum. We obtained four novel findings: 1) $\alpha$-syn impairs LTP by altering the activity of the GluN2A-containing NMDARs in SPNs belonging to the direct and indirect basal ganglia pathways; 2) $\alpha$-syn produces a reduction of NMDAR-mediated currents in SPNs optically activated by stimulation of either cortical or thalamic 
afferents; 3) the application of $\alpha$-syn-neutralizing antibodies prevents the widespread impairment of striatal LTP; 4) intrastriatal injection of $\alpha$-syn PFF produces deficits in visuo-spatial learning and GluN2A NMDA receptor function; 5) the intrastriatal injection of oligomers ( $\alpha$-syn OLIGO) mimics the behavioural effects produced by PFF, causing an even more severe visuo-spatial alteration. These data suggest that oligomeric/monomeric forms play a more relevant role than fibrillary forms on striatal-dependent behaviour.

\section{Striatal dysfunction as an early effect of alpha-synuclein toxicity}

The complex alterations of the basal ganglia pathways in Parkinson's disease involve degeneration of nigral DA neurons that leads to reduced striatal DA content and impaired synaptic plasticity of SPNs (Calabresi et al., 2007; Gerfen and Surmeier, 2011). These changes are accompanied by accumulation of $\alpha$-syn (Spillantini and Goedert, 2016), exerting a toxic role in Parkinson's disease in its oligomeric forms (Conway et al., 1998; Winner et al., 2011; Rockenstein et al., 2014) rather than in the fibrillar state or when clustered into LBs (Ghosh et al., 2015).

Although it has been previously reported that in the hippocampus micromolar concentrations of extracellular $\alpha$-syn oligomers are able to impair LTP and to increase basal synaptic transmission through a NMDAR-mediated action (Diogenes et al., 2012), little is known on the direct effects of $\alpha$-syn oligomers on striatal neurotransmission, a fundamental player in the regulation of voluntary motor function in physiological conditions and in Parkinson's disease. Our findings, demonstrating widespread involvement of much lower (nanomolar) concentrations of $\alpha$-syn oligomers in the alteration of synaptic transmission in striatal SPNs, are particularly relevant given the role exerted by altered forms of this protein in Parkinson's disease (Polymeropoulos et al., 1997; Spillantini et al., 1997; Vekrellis et al., 2011). These results extend our previous findings demonstrating that $\alpha$ syn oligomers is able to block LTP of striatal ChIs at lower concentrations $(3 \mathrm{nM})$ respect to that effective on SPNs in the present study $(30 \mathrm{nM})$, by selectively modulating the GluN2D-expressing 
NMDA receptors (Tozzi et al., 2016). Taken together these findings suggest that increasing concentrations of $\alpha$-syn oligomers progressively affect NMDAR-mediated synaptic plasticity on distinct neuronal populations indicating that the vulnerability to this protein is cell- and regionspecific.

\section{Distinct vulnerability of different NMDA receptor subunits to alpha-synuclein}

We found that $\alpha$-syn impairs NMDAR-dependent LTP in striatal SPNs with no detrimental effect on LTD, according to the observation that this latter form of synaptic plasticity in the striatum does not require NMDAR activation (Calabresi et al., 2007).

We observed that $\alpha$-syn affected SPNs glutamatergic transmission by selectively affecting GluN2Aexpressing NMDAR currents. This is a remarkable finding since NMDAR bearing the GluN2A receptor subunit is necessary for LTP induction (Paoletti et al., 2013). Accordingly, here we have shown that activity-dependent LTP in SPNs is blocked by antagonists acting on the GluN2A, but not GluN2B, NMDAR subunit. In line with these findings, our data rule out significant effects of $\alpha$ syn on currents mediated by GluN2B subunit of NMDAR and by AMPAR. Moreover, as evidenced by WB assay, $\alpha$-syn is able to lower the synaptic localization of GluN2A NMDAR subunit in striatal slices without affecting levels of other subunits of ionotropic glutamate receptors.

\section{Alpha-synuclein alters NMDAR-mediated excitatory transmission at SPNs of both direct and indirect pathways}

According to the classical view, striato-nigral SPNs express D1-like DA receptors, representing the direct pathway of the basal ganglia, while striato-pallidal SPNs express the D2-like DA receptors, representing neurons of the indirect pathway (Gerfen et al., 1990; Gerfen and Surmeier, 2011). This differential molecular expression of DA receptors is important for two functional reasons. Firstly, activation of D1 DA receptor signalling is required for LTP (Reynolds et al., 2001; Calabresi et al., 
2007), while D2 DA receptors are mainly implicated in LTD (Kreitzer and Malenka, 2007; Shen et al., 2008). The second aspect regards their possible different role in the motor function. In fact, activation of the direct pathway by DA is thought to promote movement initiation, whereas the activation of the indirect pathway would be involved in inhibition of motor activity (Mink, 2003; Nambu, 2008; Gerfen and Surmeier, 2011). In apparent contrast with this dichotomous view, more recent studies propose a new vision of the basal ganglia functionality, based on signalling integration at different levels (Cui et al., 2013; Calabresi et al., 2014). Anatomical evidences reported the existence of "bridging collaterals", axon collaterals connecting neurons of the direct and indirect pathways (Cazorla et al., 2015). On the basis of these different theories, using striatal slices from dtTomato mice, we investigated whether the alteration of LTP caused by $\alpha$-syn occurs either in a single or in both sub-populations of SPNs. We found that the detrimental effect of $\alpha$-syn on LTP and on NMDAR synaptic currents is evident both in D1- and D2-expressing SPNs.

\section{Cortico-striatal and thalamo-striatal glutamatergic inputs are equally affected by alpha-} synuclein

Further evidence of the widespread toxic effect of $\alpha$-syn on striatal synapses come from our investigation of the NMDAR-mediated current in cortico-striatal and in thalamo-striatal pathways. The striatum receives glutamatergic inputs both from cerebral cortex and thalamus (McGeorge and Faull, 1989; Smith et al., 2004; Doig et al., 2010) and these two systems act in concert to regulate motor function differentially contributing to specific tasks. Cortical afferents projecting to the dorsal striatum are mainly involved in planning, learning and execution of motor behaviour (Jog et al., 1999), whereas the thalamo-striatal inputs modulate attention-related stimuli, being engaged in behavioural switching, reinforcement functions and attention set-shifting (Smith et al., 2011). Moreover, these two systems might differentially contribute, in Parkinson's disease patients, to the generation of glutamatergic dysfunction in the striatum. Thus, we have investigated a possible 
differential effect of $\alpha$-syn on cortico-striatal versus thalamo-striatal synapses. To this aim we used a distinct optical stimulation of the two pathways using para-horizontal brain slices. Compared to coronal slices, this particular tissue preparation allows to specifically discriminating the two projections converging on SPNs (Smeal et al., 2007). Interestingly, $\alpha$-syn caused a similar reduction of GluN2A NMDAR current evoked by selectively activating either cortical or thalamic fibres. This finding, together with previous experiments performed with electrical stimulation, supports the idea that $\alpha$-syn causes a widespread alteration of excitatory synaptic transmission within the striatum.

The widespread striatal synaptic dysfunction can be prevented by antibodies against alphasynuclein

Several studies described the consequences of the application of Abs against $\alpha$-syn, reporting improvement in intracellular $\alpha$-syn aggregates clearing, reduction of $\alpha$-syn fibrils propagation, together with attenuation of memory and neurodegenerative deficits (Spencer et al., 2017). In the attempt to clarify whether specific monoclonal Abs against $\alpha$-syn exert protective effects over synaptic plasticity, we employed Syn211 Ab and SynO2 Ab; both Abs recognizing all forms of human $\alpha$-syn, however, SynO2 Ab is much more potent than Syn211 Ab in binding $\alpha$-syn oligomers and fibrils (Giasson et al., 2000; Majbour et al., 2016). The depletion experiment we performed, confirmed that either Syn211 Ab or SynO2 Ab shows affinity for $\alpha$-syn oligomeric aggregates, with a different binding specificity between the two Abs. Interestingly, we found that, an overlapping dose-response effect of the two Abs in rescuing the LTP impairment induced by $\alpha$ syn in striatal SPNs. The two Abs also prevented the reduction of GluN2A expression levels caused by $\alpha$-syn. This protective effect is consistent with our observation that $\alpha$-syn alters LTP affecting NMDAR-mediated currents, in particular the GluN2A subunit. Interestingly, the PFF preparation injected intrastriatally in in vivo experiments contained both OLIGO and PFF aggregates. 


\section{Differential effect of fibrillar versus oligomeric forms of alpha-synuclein on visuo-spatial} learning

Accumulating in vivo evidence showed that $\alpha$-syn protofibrils directly injected in the striatum lead to a time-dependent spreading all over the brain accompanied by neuronal loss and motor deficits in mice within 9 months after the injection (Luk et al., 2012). In this study we aimed at verifying whether the pathological interaction between $\alpha$-syn aggregates and NMDA receptors in the striatum led to impaired behavioural function; for this reason, we focused on an early time point when the aggregates remained localized within the striatum. To dissect the behavioural effects of $\alpha$-syn aggregates we used a visuo-spatial learning task that we have previously shown to rely on NMDA receptors activation in the dorsal striatum (De Leonibus et al., 2003; De Leonibus et al., 2005). $\alpha$ syn PFF injected rats show impaired spatial novelty detection, although they normally explore the objects during the habituation phase and can discriminate a new object among familiar ones.

Interestingly, the intrastriatal injection of oligomers mimics the behavioural effects produced by PFF. This effect is even more severe than that one induced by PFF injection since while fibrils only affect the ability to discriminate the object displacement, oligomers impair both spatial and object novelty recognition. This is surprising considering that the PFF preparation injected intrastriatally in in vivo experiments contained both OLIGO and PFF aggregates and that the $\alpha$-syn cocktail producing the rapid synaptic and plastic alterations mainly contain oligomeric/monomeric forms, but little or no fibrils, as confirmed by the ThT analyses.

While the effect of in vivo injection of $\alpha$-syn on SPN NMDAR currents does not provide a direct mechanistic explanation that NMDAR changes are responsible of the described behavioural alteration, it confirms that $\alpha$-syn is able to produce molecular changes affecting the function of this receptor with possible behavioural consequences. 
Our experiments in $\alpha$-syn-injected rats showed specific deficits in visuo-spatial exploration while locomotor activity was not affected respect to control animals. It possible that we failed to detect motor deficits because of the site and/or size of the injection within the striatum.

All together, these findings suggest that oligomeric $\alpha$-syn itself is harmful for synaptic plasticity and behavioural functions of the dorsal striatum. We speculate that further $\alpha$-syn aggregation into protofibrils might limit their direct impact within the area of injection, while favouring their spreading across brain regions as previously reported (Luk et al., 2012). Further studies are necessary to address this issue. The translational relevance of this preclinical data for PD are evidenced by findings in humans showing visuo-spatial learning deficit in de novo PD patients showing spatial location deficits (Pillon et al., 1997).

\section{Conclusions}

Although the role of $\alpha$-syn in the pathogenesis of Parkinson's disease has been deeply investigated, little is known on the effect of $\alpha$-syn on striatal synaptic transmission and related early behavioural alterations. Our research demonstrates a widespread and NMDAR-related dysfunction of striatal synaptic network induced by $\alpha$-syn. We report for the first time that $\alpha$-syn, through interaction with GluN2A-bearing NMDAR, impairs LTP in SPNs of the direct and indirect basal ganglia pathways and causes visuo-spatial learning deficit. The $\alpha$-syn-induced synaptopathy is also observed when neurons are optogenetically activated by either cortical or thalamic inputs. Notably, both plastic and behavioural alterations observed in our study do not seem to be dependent on dopaminergic degeneration. In fact, the plastic dysfunctions induced by acute in vitro application of $\alpha$-syn are not rescued by exogenous DA. Accordingly, the observed visuo-spatial learning deficits are not associated with a loss of dopaminergic terminals. Moreover, the open field analysis does not show overt motor impairment indicating that our in vivo model recapitulates an early stage of the disease. 
Finally, we provide evidence that antibodies neutralizing $\alpha$-syn are effective in blocking its detrimental actions. Taken together these data support the use of antibodies against $\alpha$-syn as a valid approach to preserve the functionality of synaptic plasticity in the striatal network.

FINANCIAL DISCLOSURES. All authors reported no biomedical financial interests or potential conflicts of interest.

ACKNOWLEDGMENTS. This work was supported by grants from Fondazione Cariplo Grant 2014-0660 (FG and PC), MIUR-PRIN 2015FNWP34 (PC, FG and EDL), Ricerca Finalizzata RF2013-02356215 (PC and FG) and RF-2013-02357386 (BP). Valentina Durante at present has a fellowship from "Dipartimento Neuroscienze, psicologia, area del farmaco e salute del bambino, Università di Firenze", her position has been supported by "AIRAlzh Onlus-COOP Italia". This work/project was supported by a grant (V.G., P.C.) from the Fresco Parkinson Institute to New York University School of Medicine and The Marlene and Paolo Fresco Institute for Parkinson's and Movement Disorders, which was made possible with support from Marlene and Paolo Fresco. We thank Cristiano Spaccatini, Nadia Santo and Elisa Zianni for their excellent technical support.

SUPPLEMENTARY MATERIAL. Supplementary material is available at Brain online.

\section{REFERENCES}

Abeliovich A, Schmitz Y, Farinas I, Choi-Lundberg D, Ho WH, Castillo PE, et al. Mice lacking alpha-synuclein display functional deficits in the nigrostriatal dopamine system. Neuron 2000; 25(1): 239-52.

Arenkiel BR, Peca J, Davison IG, Feliciano C, Deisseroth K, Augustine GJ, et al. In vivo lightinduced activation of neural circuitry in transgenic mice expressing channelrhodopsin-2. Neuron 2007; 54(2): 205-18.

Cabin DE, Shimazu K, Murphy D, Cole NB, Gottschalk W, McIlwain KL, et al. Synaptic vesicle depletion correlates with attenuated synaptic responses to prolonged repetitive stimulation in mice lacking alpha-synuclein. J Neurosci 2002; 22(20): 8797-807. 
Calabresi P, Maj R, Pisani A, Mercuri NB, Bernardi G. Long-term synaptic depression in the striatum: physiological and pharmacological characterization. J Neurosci 1992a; 12(11): 422433.

Calabresi P, Picconi B, Tozzi A, Di Filippo M. Dopamine-mediated regulation of corticostriatal synaptic plasticity. Trends Neurosci 2007; 30(5): 211-9.

Calabresi P, Picconi B, Tozzi A, Ghiglieri V, Di Filippo M. Direct and indirect pathways of basal ganglia: a critical reappraisal. Nat Neurosci 2014; 17(8): 1022-30.

Calabresi P, Pisani A, Mercuri NB, Bernardi G. Long-term Potentiation in the Striatum is Unmasked by Removing the Voltage-dependent Magnesium Block of NMDA Receptor Channels. Eur J Neurosci 1992b; 4(10): 929-35.

Cazorla M, Kang UJ, Kellendonk C. Balancing the basal ganglia circuitry: a possible new role for dopamine D2 receptors in health and disease. Mov Disord 2015; 30(7): 895-903.

Conway KA, Harper JD, Lansbury PT. Accelerated in vitro fibril formation by a mutant alphasynuclein linked to early-onset Parkinson disease. Nat Med 1998; 4(11): 1318-20.

Cui G, Jun SB, Jin X, Pham MD, Vogel SS, Lovinger DM, et al. Concurrent activation of striatal direct and indirect pathways during action initiation. Nature 2013; 494(7436): 238-42.

De Leonibus E, Lafenetre P, Oliverio A, Mele A. Pharmacological evidence of the role of dorsal striatum in spatial memory consolidation in mice. Behav Neurosci 2003; 117(4): 685-94.

De Leonibus E, Manago F, Giordani F, Petrosino F, Lopez S, Oliverio A, et al. Metabotropic glutamate receptors 5 blockade reverses spatial memory deficits in a mouse model of Parkinson's disease. Neuropsychopharmacology 2009; 34(3): 729-38.

De Leonibus E, Oliverio A, Mele A. A study on the role of the dorsal striatum and the nucleus accumbens in allocentric and egocentric spatial memory consolidation. Learn Mem 2005; 12(5): 491-503.

De Leonibus E, Pascucci T, Lopez S, Oliverio A, Amalric M, Mele A. Spatial deficits in a mouse model of Parkinson disease. Psychopharmacology (Berl) 2007; 194(4): 517-25.

Ding J, Peterson JD, Surmeier DJ. Corticostriatal and thalamostriatal synapses have distinctive properties. J Neurosci 2008; 28(25): 6483-92.

Diogenes MJ, Dias RB, Rombo DM, Vicente Miranda H, Maiolino F, Guerreiro P, et al. Extracellular alpha-synuclein oligomers modulate synaptic transmission and impair LTP via NMDA-receptor activation. J Neurosci 2012; 32(34): 11750-62.

Doig NM, Moss J, Bolam JP. Cortical and thalamic innervation of direct and indirect pathway medium-sized spiny neurons in mouse striatum. J Neurosci 2010; 30(44): 14610-8. 
Dunah AW, Wang Y, Yasuda RP, Kameyama K, Huganir RL, Wolfe BB, et al. Alterations in subunit expression, composition, and phosphorylation of striatal N-methyl-D-aspartate glutamate receptors in a rat 6-hydroxydopamine model of Parkinson's disease. Mol Pharmacol 2000; 57(2): 342-52.

El-Agnaf OM, Salem SA, Paleologou KE, Cooper LJ, Fullwood NJ, Gibson MJ, et al. Alphasynuclein implicated in Parkinson's disease is present in extracellular biological fluids, including human plasma. FASEB J 2003; 17(13): 1945-7.

Gallegos S, Pacheco C, Peters C, Opazo CM, Aguayo LG. Features of alpha-synuclein that could explain the progression and irreversibility of Parkinson's disease. Front Neurosci 2015; 9: 59.

Gardoni F, Picconi B, Ghiglieri V, Polli F, Bagetta V, Bernardi G, et al. A critical interaction between NR2B and MAGUK in L-DOPA induced dyskinesia. J Neurosci 2006; 26(11): 291422.

Gerfen CR, Engber TM, Mahan LC, Susel Z, Chase TN, Monsma FJ, Jr., et al. D1 and D2 dopamine receptor-regulated gene expression of striatonigral and striatopallidal neurons. Science 1990; 250(4986): 1429-32.

Gerfen CR, Surmeier DJ. Modulation of striatal projection systems by dopamine. Annu Rev Neurosci 2011; 34: 441-66.

Ghiglieri V, Calabrese V, Calabresi P. Alpha-Synuclein: From Early Synaptic Dysfunction to Neurodegeneration. Front Neurol 2018; 9: 295.

Ghosh D, Singh PK, Sahay S, Jha NN, Jacob RS, Sen S, et al. Structure based aggregation studies reveal the presence of helix-rich intermediate during alpha-Synuclein aggregation. Sci Rep 2015; 5: 9228.

Giasson BI, Jakes R, Goedert M, Duda JE, Leight S, Trojanowski JQ, et al. A panel of epitopespecific antibodies detects protein domains distributed throughout human alpha-synuclein in Lewy bodies of Parkinson's disease. J Neurosci Res 2000; 59(4): 528-33.

Jocoy EL, Andre VM, Cummings DM, Rao SP, Wu N, Ramsey AJ, et al. Dissecting the contribution of individual receptor subunits to the enhancement of N-methyl-d-aspartate currents by dopamine D1 receptor activation in striatum. Front Syst Neurosci 2011; 5: 28.

Jog MS, Kubota Y, Connolly CI, Hillegaart V, Graybiel AM. Building neural representations of habits. Science 1999; 286(5445): 1745-9.

Kirik D, Rosenblad C, Bjorklund A. Characterization of behavioral and neurodegenerative changes following partial lesions of the nigrostriatal dopamine system induced by intrastriatal 6hydroxydopamine in the rat. Exp Neurol 1998; 152(2): 259-77. 
Kreitzer AC, Malenka RC. Endocannabinoid-mediated rescue of striatal LTD and motor deficits in Parkinson's disease models. Nature 2007; 445(7128): 643-7.

Lim DH, Mohajerani MH, Ledue J, Boyd J, Chen S, Murphy TH. In vivo Large-Scale Cortical Mapping Using Channelrhodopsin-2 Stimulation in Transgenic Mice Reveals Asymmetric and Reciprocal Relationships between Cortical Areas. Front Neural Circuits 2012; 6: 11.

Luk KC, Kehm V, Carroll J, Zhang B, O'Brien P, Trojanowski JQ, et al. Pathological alphasynuclein transmission initiates Parkinson-like neurodegeneration in nontransgenic mice. Science 2012; 338(6109): 949-53.

Majbour NK, Vaikath NN, van Dijk KD, Ardah MT, Varghese S, Vesterager LB, et al. Oligomeric and phosphorylated alpha-synuclein as potential CSF biomarkers for Parkinson's disease. Mol Neurodegener 2016; 11: 7.

McGeorge AJ, Faull RL. The organization of the projection from the cerebral cortex to the striatum in the rat. Neuroscience 1989; 29(3): 503-37.

Mellone M, Stanic J, Hernandez LF, Iglesias E, Zianni E, Longhi A, et al. NMDA receptor GluN2A/GluN2B subunit ratio as synaptic trait of levodopa-induced dyskinesias: from experimental models to patients. Front Cell Neurosci 2015; 9: 245.

Mink JW. The Basal Ganglia and involuntary movements: impaired inhibition of competing motor patterns. Arch Neurol 2003; 60(10): 1365-8.

Nambu A. Seven problems on the basal ganglia. Curr Opin Neurobiol 2008; 18(6): 595-604.

Nilsson MR. Techniques to study amyloid fibril formation in vitro. Methods 2004; 34(1): 151-60.

Paoletti P, Bellone C, Zhou Q. NMDA receptor subunit diversity: impact on receptor properties, synaptic plasticity and disease. Nat Rev Neurosci 2013; 14(6): 383-400.

Paxinos GW, C. . The Rat Brain Stereotaxic Coordinates. 5th Edn San Diego. Elsevier Academic Press 2005 .

Pillon B, Ertle S, Deweer B, Bonnet AM, Vidailhet M, Dubois B. Memory for spatial location in 'de novo' parkinsonian patients. Neuropsychologia 1997; 35(3): 221-8.

Polymeropoulos MH, Lavedan C, Leroy E, Ide SE, Dehejia A, Dutra A, et al. Mutation in the alpha-synuclein gene identified in families with Parkinson's disease. Science 1997; 276(5321): 2045-7.

Reynolds JN, Hyland BI, Wickens JR. A cellular mechanism of reward-related learning. Nature $2001 ; 413(6851): 67-70$. 
Rockenstein E, Nuber S, Overk CR, Ubhi K, Mante M, Patrick C, et al. Accumulation of oligomerprone alpha-synuclein exacerbates synaptic and neuronal degeneration in vivo. Brain 2014; 137(Pt 5): 1496-513.

Rodo C, Sargolini F, Save E. Processing of spatial and non-spatial information in rats with lesions of the medial and lateral entorhinal cortex: Environmental complexity matters. Behav Brain Res 2017; 320: 200-9.

Royston P. A Toolkit for Testing for Non-Normality in Complete and Censored Samples. Journal of the Royal Statistical Society Series D (The Statistician) 1993; 42(1): 37-43.

Sciamanna G, Tassone A, Mandolesi G, Puglisi F, Ponterio G, Martella G, et al. Cholinergic dysfunction alters synaptic integration between thalamostriatal and corticostriatal inputs in DYT1 dystonia. J Neurosci 2012; 32(35): 11991-2004.

Shen W, Flajolet M, Greengard P, Surmeier DJ. Dichotomous dopaminergic control of striatal synaptic plasticity. Science 2008; 321(5890): 848-51.

Smeal RM, Gaspar RC, Keefe KA, Wilcox KS. A rat brain slice preparation for characterizing both thalamostriatal and corticostriatal afferents. J Neurosci Methods 2007; 159(2): 224-35.

Smith Y, Raju DV, Pare JF, Sidibe M. The thalamostriatal system: a highly specific network of the basal ganglia circuitry. Trends Neurosci 2004; 27(9): 520-7.

Smith Y, Surmeier DJ, Redgrave P, Kimura M. Thalamic contributions to Basal Ganglia-related behavioral switching and reinforcement. J Neurosci 2011; 31(45): 16102-6.

Spencer B, Valera E, Rockenstein E, Overk C, Mante M, Adame A, et al. Anti-alpha-synuclein immunotherapy reduces alpha-synuclein propagation in the axon and degeneration in a combined viral vector and transgenic model of synucleinopathy. Acta Neuropathol Commun 2017; 5(1): 7 .

Spillantini MG, Goedert M. Synucleinopathies: past, present and future. Neuropathol Appl Neurobiol 2016; 42(1): 3-5.

Spillantini MG, Schmidt ML, Lee VM, Trojanowski JQ, Jakes R, Goedert M. Alpha-synuclein in Lewy bodies. Nature 1997; 388(6645): 839-40.

Tanaka M, Kim YM, Lee G, Junn E, Iwatsubo T, Mouradian MM. Aggresomes formed by alphasynuclein and synphilin-1 are cytoprotective. J Biol Chem 2004; 279(6): 4625-31.

Tozzi A, de Iure A, Bagetta V, Tantucci M, Durante V, Quiroga-Varela A, et al. Alpha-Synuclein Produces Early Behavioral Alterations via Striatal Cholinergic Synaptic Dysfunction by Interacting With GluN2D N-Methyl-D-Aspartate Receptor Subunit. Biol Psychiatry 2016; 79(5): 402-14. 
Vaikath NN, Majbour NK, Paleologou KE, Ardah MT, van Dam E, van de Berg WD, et al. Generation and characterization of novel conformation-specific monoclonal antibodies for alpha-synuclein pathology. Neurobiol Dis 2015; 79: 81-99.

Vastagh C, Gardoni F, Bagetta V, Stanic J, Zianni E, Giampa C, et al. N-methyl-D-aspartate (NMDA) receptor composition modulates dendritic spine morphology in striatal medium spiny neurons. J Biol Chem 2012; 287(22): 18103-14.

Vekrellis K, Xilouri M, Emmanouilidou E, Rideout HJ, Stefanis L. Pathological roles of alphasynuclein in neurological disorders. Lancet Neurol 2011; 10(11): 1015-25.

Winner B, Jappelli R, Maji SK, Desplats PA, Boyer L, Aigner S, et al. In vivo demonstration that alpha-synuclein oligomers are toxic. Proc Natl Acad Sci U S A 2011; 108(10): 4194-9.

Wong YC, Krainc D. alpha-synuclein toxicity in neurodegeneration: mechanism and therapeutic strategies. Nat Med 2017; 23(2): 1-13.

Wu YW, Kim JI, Tawfik VL, Lalchandani RR, Scherrer G, Ding JB. Input- and cell-type-specific endocannabinoid-dependent LTD in the striatum. Cell Rep 2015; 10(1): 75-87.

\section{Figure legends}

Figure 1. Effect of $\alpha$-synuclein on the intrinsic membrane properties and synaptic plasticity of

striatal SPNs. (A) Representative voltage traces (top) and current-voltage graph (bottom) recorded from SPNs during the injection of hyperpolarizing and depolarizing steps of current in control conditions ( $n=5)$ and in the presence of $30 \mathrm{nM} \alpha$-syn (n=4) show no differences in the firing pattern discharge and intrinsic membrane properties of these neurons. (B) Examples traces (top) and time course (bottom) of the excitatory postsynaptic currents (EPSCs) measured before and 30 minutes after the application of the HFS protocol show no differences in the LTD induction in SPNs recorded from control slices $(n=6)$ and from slices incubated with $\alpha$-syn $(n=6)$. (C) Paired EPSC traces (top) and time-course graph of the EPSC amplitude (bottom) recorded in SPNs from control slices $(n=7)$ and in SPNs of slices incubated with $30 \mathrm{nM} \alpha$-syn $(\mathrm{n}=7)$ before and after the delivery of the HFS protocol show the block of LTP in SPNs recorded from slices treated with $\alpha$-syn. (D) 
Histogram showing the EPSC amplitude 30 min following the HFS protocol of SPNs recorded from control striatal slices and from slices incubated with increasing concentrations of $\alpha$-syn $(3 \mathrm{nM}, \mathrm{n}=6$, $10 \mathrm{nM}, \mathrm{n}=7,300 \mathrm{nM}, \mathrm{n}=6$ ). (E) EPSC traces (top) and time-course graph of the EPSC amplitude (bottom) measured in SPNs of control slices $(\mathrm{n}=7)$ and of slices incubated with $30 \mathrm{nM} \alpha$-syn in the presence of $30 \mu \mathrm{M}$ dopamine (DA) (n=6) bath applied before and after the HFS protocol. Data are represented as mean \pm SEM. (F) Histogram of the ELISA shows time- and dose-dependent $\alpha$-syn oligomers; higher level of $\alpha$-syn oligomers were detected in the $300 \mathrm{nM} \alpha$-syn samples collected at 30 min. (G) ThT assay shows that little fluorescence was detected with the $30 \mathrm{nM}$ concentration, independently on the timing of sampling. In contrast, high fluorescence signal was measurable with the $300 \mathrm{nM}$ dose. Data are reported as percentage of fluorescence registered at $\mathrm{T} 0 \pm \mathrm{SEM} . * \mathrm{P}<0.05$, $* * \mathrm{P}<0.01, * * * \mathrm{P}<0.001$

Figure 2. Effect of alpha-synuclein on AMPAR- and NMDAR-mediated currents. (A) Example traces (top) and histogram (bottom) showing the AMPAR-mediated synaptic current of SPNs recorded in pharmacological isolation, in the presence of APV and picrotoxin, before and after the application of $30 \mathrm{nM} \alpha$-syn (n=6). (B) Example traces of EPSCs (top) evoked at +40 and $-80 \mathrm{mV}$ holding potential and histogram (bottom) showing the AMPAR RI of SPNs recorded in control conditions and in the presence of $\alpha$-syn $(n=6)$. (C) Histogram showing the averaged EPSC amplitude carried by the GluN2A-expressing NMDAR measured in SPNs in the presence of picrotoxin, CNQX and ifenprodil (open orange bar) and after subsequent addition of $30 \mathrm{nM} \alpha$-syn $(n=6)$ (filled cyan bar). The superimposed traces show EPSCs acquired at $+40 \mathrm{mV}$ holding potential of a SPN in the presence of picrotoxin + CNQX (black trace), picrotoxin + CNQX + ifenprodil (orange trace) and picrotoxin + CNQX + ifenprodil $+\alpha$-syn (cyan trace). (D, E) Histograms showing the averaged EPSC amplitudes carried by the GluN2B-expressing NMDAR measured in SPNs in the presence of picrotoxin, CNQX and NVP-AAM077 ( $\mathrm{n}=6)$ (D, open violet bar) or 
picrotoxin, CNQX and TCN-201 (n=6) (E, open violet bar) and in the presence of $30 \mathrm{nM} \alpha$-syn on this current $(n=6)$ (D-E, filled cyan bars). Superimposed traces showing EPSCs acquired from a single SPN following the application of picrotoxin + CNQX (D, black trace), picrotoxin + CNQX + NVP-AAM077 (D, violet trace), picrotoxin + CNQX + NVP-AAM077 + $\alpha$-syn (D, cyan trace) or picrotoxin + CNQX (E, black trace), picrotoxin + CNQX + TCN-201 (E, violet trace), picrotoxin + CNQX + TCN-201 $+\alpha$-syn $(\mathrm{E}$, cyan trace). (F, G) Representative WB images and histograms showing the effects of incubation (1 hour) of corticostriatal slices with $30 \mathrm{nM} \alpha$-syn on the synaptic localization of GluA1 and GluA2 AMPAR subunits $(\mathrm{n}=8)(\mathrm{F})$ and GluN2A and GluN2B NMDAR subunits $(n=6)(G)$. Data are normalized against tubulin and are shown in the graph as mean \pm SEM. $* \mathrm{P}<0.05, * * \mathrm{P}<0.01$.

\section{Figure 3. Effect of alpha-synuclein on SPNs of the direct and indirect basal ganglia pathway.}

(A) Images of three regions acquired from a striatal slice of a transgenic Drdla-tdTomato mouse showing fluorescent D1R-positive neurons (left, in red pseudocolor and top right) and a nonfluorescent (putative D2R-positive) SPN (bottom right). (B) Example traces and time-course graph showing the LTP induced in both fluorescent $(n=6)$ and non-fluorescent $(n=6)$ SPNs after the delivery of the HFS protocol. (C, D) EPSC pairs (top) and time-courses graphs of the EPSC amplitude (bottom) showing that $\alpha$-syn is able to block the LTP in both D1R-positive (n=5) (C) and putative D2R-positive SPNs (n=5) (D) following the HFS protocol. (E, F) Histograms showing the mean EPSC amplitudes of the NMDAR-mediated synaptic currents measured in pharmacological isolation in D1R-positive $(n=6)(E)$ and in putative D2R-positive SPNs $(n=4)(F)$ both in control condition and after the application of $30 \mathrm{nM} \alpha$-syn. The superimposed traces show EPSCs acquired from a D1R-positive SPN (E) and putative D2R-positive SPN (F) in the presence of CNQX plus picrotoxin and in CNQX plus picrotoxin plus $\alpha$-syn. Note that $\alpha$-syn is able to reduce the NMDAR- 
mediated current in a similar manner in these neuronal sub-populations. Data are represented as mean \pm SEM. $* \mathrm{P}<0.05, * * \mathrm{P}<0.01$

Figure 4. Effect of alpha-synuclein on SPNs activated by optical stimulation of cortico-striatal and thalamo-striatal glutamatergic afferents. (A) Representative para-horizontal brain slice including cortical, striatal and thalamic regions and (B) scheme of a para-horizontal slice showing the regions of the cortex and the thalamus where optical stimulation can activate glutamatergic afferents to SPNs recorded in the striatum. (C, F) Histograms of the SPNs mean NMDA-EPSC amplitudes optically stimulated in the cortex (C) or in the thalamus (F) in the presence of $3 \mu \mathrm{M}$ ifenprodil to isolate the GluN2A-EPSC ( $n=6$ cortex, $n=5$ thalamus) (orange open bar) or in the presence of $10 \mu \mathrm{M}$ TCN-201 $(n=6)$ to isolate the GluN2B-EPSC (violet open bar). Data are presented as percentage of the control NMDAR-EPSCs recorded in the presence of picrotoxin and CNQX (dotted line). (D, G) Histograms of the averaged GluN2A- and GluN2B-EPSC amplitudes optically stimulated in the cortex (D) or in the thalamus (G) measured in SPNs in the presence of picrotoxin, CNQX, ifenprodil (orange outlined bars) without $(\mathrm{n}=6)$ and with $30 \mathrm{nM} \alpha$-syn (orange outlined, cyan filled bar) and picrotoxin, CNQX, TCN-201 (violet outlined bars) without $(\mathrm{n}=6)$ and with $30 \mathrm{nM} \alpha$-syn (violet outlined, cyan filled bar). $\alpha$-syn (n=4-6) is applied for 2, 5 and $10 \mathrm{~min}$. Data are presented as percentage of the control GluN2A- and GluN2B-EPSCs. (E, H) Top, superimposed EPSCs evoked by optical stimulation of the cortex $(\mathrm{E})$ or the thalamus $(\mathrm{H})$ acquired from a SPN in the presence of picrotoxin, CNQX, ifenprodil (orange trace) and after further the application of $30 \mathrm{nM} \alpha$-syn (cyan trace). Bottom, EPSCs acquired from a SPN in the presence of picrotoxin, CNQX, TCN-201 (violet trace) and after further the application of $30 \mathrm{nM} \alpha$-syn (cyan trace). Note that $\alpha$-syn significantly reduces the GluN2A- but not GluN2B-EPSC. Data are represented as mean $\pm \mathrm{SEM} . * * \mathrm{P}<0.01, * * * \mathrm{P}<0.001$. 
Figure 5. Action of monoclonal antibodies against $\alpha$-synuclein on SPNs synaptic plasticity. (A) Time-course graph showing the EPSC amplitudes of SPNs recorded before and after the LTP induction by HFS in slices incubated for 1 hour with $30 \mathrm{nM} \alpha$-syn and in slices treated with Syn211 Ab (1:10) alone $(n=7)$ or with Syn211 Ab (1:10) plus $\alpha$-syn $(n=7)$. (B) Time-course of the SPNs EPSC amplitudes recorded before and after the HFS protocol in slices treated with $\alpha$-syn and in SPNs in the presence of SynO2 Ab alone (1:10.000) $(n=7)$ or SynO2 Ab (1:10.000) plus $\alpha$-syn $(n=7)$. Note that the treatment with Syn211 or SynO2 Abs is able to restore the LTP in the presence of $\alpha$-syn. (C) Immunoprecipitation experiment with the samples collected at time 0 and 30 min with antibodies (SynO2 or Syn211) estimates the $\alpha$-syn oligomers left in the sample using oligomeric ELISA. (D, E) WB analysis for GluN2A subunit of the NMDAR in the postsynaptic fraction (TIF) obtained from striatal slices incubated with $\alpha$-syn alone (30 nM) or with $\alpha$-syn plus Syn211 (1:10) or Syn-O2 (1:10.000) antibodies and control slices. $\alpha$-syn reduced GluN2A localization at postsynaptic sites, while treatment with two different anti- $\alpha$-syn antibodies was able to rescue GluN2A levels in the TIF. Data are normalized against tubulin and are shown in the graph as mean \pm SEM. $* \mathrm{P}<0.05, * * \mathrm{P}<0.01$

Figure 6. Intra-striatal PFF alpha-synuclein injection. (A) Representative TEM image of preformed fibrils (PFF) preparation injected in rat striatum. $(\mathbf{B}, \mathbf{C})$ Representative brain slice of animals injected with $\alpha$-syn in the striatum. Immunofluorescence for $\alpha$-syn and phospho-Ser129-hu$\alpha$-Syn. High levels of phosphor-Ser129-hu- $\alpha$-Syn are detectable at the injection site within the striatum, with no spreading in the surrounding brain regions. (B) A higher magnification (60x) evidences the $\alpha$-syn aggregates positive for phospho-Ser129hu- $\alpha$-Syn. (B) Representative brain slice of animals injected with PBS in the striatum. No positive staining of phospho-Ser129-hu- $\alpha-$ Syn is detectable in the striatum of the control group at low and high magnification (C). 


\section{Figure 7. Behavioural and electrophysiological alterations in rats injected with human alpha-}

synuclein. (A) Schematic representation of the visuo-spatial novelty reaction task. Rats were habituated to the empty arena (S1). During the habituation phase they were allowed to explore for 6 consecutive sessions (S2-S7) three different objects kept in constant position. Spatial learning was tested by displacing the position of two objects, the blu one (Displaced object-DO), and comparing its relative preference as compared to the non-displaced objects (NDO), the black and the purple once (S8). Animals were given a further session to habituate to the spatial change (S9). During the last session object learning was tested by substituting one of the three objects (the blu one, substituted object-SO) with a new one (orange), and the other two objects with identical copies, to avoid the use of olfactory cues for object recognition (non-substituted objects-NSO) (S10). (B) Histograms showing the mean time (s) objects exploration during the habituation phase by control and $\alpha$-syn rats (left panel); Re-exploration index of displaced DO and NDO objects after the spatial change, showing that control, but not $\alpha$-syn rats, prefer DO vs. NDO (center panel); Re-exploration index of substituted SO and NSO objects after the object change, showing that both control and $\alpha$ syn rats preferred SO vs. NSO (right panel). ${ }^{*} \mathrm{P}<0.05 \mathrm{DO} / \mathrm{SO}$ vs. NDO/NSO, within group; \# $\mathrm{P}<0.05 \alpha$-syn vs. control, Duncan post-hoc test. DO: displaced objects; NDO: non-displaced objects; SO: substituted object; NSO: non-substituted object. Control rats, $n=9, \alpha$-syn rats, $n=8$. Histogram showing the NMDA/AMPA ratio measured in SPNs of $\alpha$-syn- $(n=5)$ and of shaminjected (control) rats $(\mathrm{n}=5)$. Traces showing an EPSC measured at $+40 \mathrm{mV}$ and $-80 \mathrm{mV}$ holding potential in a SPN of a control and $\alpha$-syn rat. (D) Histogram showing the NMDA-current amplitude of SPNs from $\alpha$-syn- $(n=5)$ and control rats $(n=5)$ before (dashed line) and after the application of ifenprodil to isolate the GluN2A-mediated NMDA-current. Traces showing an EPSC measured at $+40 \mathrm{mV}$ holding potential in a SPN of a control and $\alpha$-syn rat before and after the application of ifenprodil. * $\mathrm{P}<0.05, * * \mathrm{P}<0.01$ 
Figure 8. Intra-striatal oligomeric alpha-synuclein injection and behavioural alterations. (A) Representative TEM image of OLIGO $\alpha$-syn preparation injected in rat striatum showing a majority of small oligomers with very few protofibrils. (B-C) Representative images of animals injected with $\alpha$-syn in the striatum. Immunofluorescence for hu- $\alpha$-syn (B) and phospho-Ser129-hu- $\alpha$-Syn (C). 40x magnification images evidences that high levels of hu- $\alpha$-syn (B) and phosphor-Ser129-hu- $\alpha$ Syn (C) are detectable within the striatum. Representative images of animals injected with PBS in the striatum (B, C). No positive staining of phospho-Ser129-hu- $\alpha-S y n$ (C) is detectable in the striatum of the control group at 40x magnification. Arrows indicate hu- $\alpha$-syn and phospho-Ser129hu- $\alpha$-Syn positive cells; scale bars: $50 \mu \mathrm{m}$. (D-F) Histograms showing the mean time (s) objects exploration during the habituation phase by control and oligomeric $\alpha$-syn rats. Re-exploration index of displaced DO and NDO objects after the spatial change, showing that control, but not $\alpha$-syn rats, prefer DO vs. NDO (center panel). Re-exploration index of substituted SO and NSO objects after the object change, showing that both control and $\alpha$-syn rats preferred SO vs. NSO (right panel). Control rats, $\mathrm{n}=6$, oligomeric $\alpha$-syn rats, $\mathrm{n}=6$. ${ }^{*} \mathrm{P}<0.05 \mathrm{DO} / \mathrm{SO}$ vs. NDO/NSO, within group, Duncan post-hoc test. DO: displaced objects; NDO: non-displaced objects; SO: substituted object; NSO: non-substituted object. 


\section{Alpha-synuclein targets GluN2A NMDA receptor subunit causing striatal synaptic dysfunction and visuo-spatial memory alteration}

Durante $\mathrm{V}^{1}$, de Iure $\mathrm{A}^{1,2}$, Loffredo $\mathrm{V}^{3,4}$, Vaikath $\mathrm{N}^{5}$, De Risi $\mathrm{M}^{6}$, Paciotti $\mathrm{S}^{7}$, Quiroga-Varela $\mathrm{A}^{1}$, Chiasserini $\mathrm{D}^{1}$, Mellone $\mathrm{M}^{8}$, Mazzocchetti $\mathrm{P}^{1}$, Calabrese $\mathrm{V}^{1}$, Campanelli $\mathrm{F}^{1,9}$, Mechelli $\mathrm{A}^{1}$, Di Filippo $\mathrm{M}^{1}$, Ghiglieri $\mathrm{V}^{9,10}$, Picconi $\mathrm{B}^{2}$, El-Agnaf $\mathrm{OM}^{5}$, De Leonibus $\mathrm{E}^{3,6}$, Gardoni $\mathrm{F}^{8}$, Tozzi $\mathrm{A}^{7,9}$, and Calabresi $\mathrm{P}^{1,9^{*}}$

1. Neurological Clinic, Department of Medicine, Hospital Santa Maria della Misericordia, University of Perugia, Perugia, Italy;

2. Laboratory of Experimental Neurophysiology, IRCCS San Raffaele Pisana, Rome, Italy

3. Institute of Cellular Biology and Neurobiology, National Research Council, Monterotondo (Rome), Italy;

4. PhD Program in Behavioral Neuroscience, Sapienza University of Rome, Italy;

5. Neurological Disorders Research Center, Qatar Biomedical Research Institute (QBRI), Hamad Bin Khalifa University (HBKU), Qatar Foundation, Doha, Qatar;

6. Telethon Institute of Genetics and Medicine, Telethon Foundation, Pozzuoli (NA), Italy;

7. Department of Experimental Medicine, Section of Physiology and Biochemistry, University of Perugia, Perugia, Italy;

8. Department of Pharmacological and Biomolecular Sciences, University of Milan, Italy;

9. Laboratory of Neurophysiology, Santa Lucia Foundation, IRCCS, Rome, Italy;

10. Department of Philosophy, Human, Social and Educational Sciences, University of Perugia, Perugia, Italy.

Correspondence to:

Professor Paolo Calabresi

Neurological Clinic, Department of Medicine, University of Perugia,

Hospital Santa Maria della Misericordia,

06132 - S. Andrea delle Fratte, Perugia, Italy

Phone +39-075-578-4230; Fax +39-075-578-4229

Email: paolo.calabresi@unipg.it

Running title: Alpha-synuclein in synaptic transmission 


\begin{abstract}
Parkinson's disease is a progressive neurodegenerative disorder characterized by altered striatal dopaminergic signalling that leads to motor and cognitive deficits. Parkinson's disease is also characterized by abnormal presence of soluble toxic forms of alpha-synuclein ( $\alpha$-syn) that, when clustered into Lewy bodies, represents one of the pathological hallmark of the disease. However, also $\alpha$-syn oligomers might directly affect synaptic transmission and plasticity in Parkinson's disease models. Accordingly, by combining electrophysiological, optogenetic, immunofluorescence, molecular and behavioural analyses, here we report that $\alpha$-syn reduces $\mathrm{N}$ methyl-D-aspartate (NMDA) receptor-mediated synaptic currents and impairs corticostriatal longterm potentiation (LTP) of striatal spiny projection neurons (SPNs), of both direct (D1-positive) and indirect (putative D2-positive) pathways. Intrastriatal injections of $\alpha$-syn produce deficits in visuospatial learning associated with reduced function of GluN2A NMDA receptor subunit indicating that this protein selectively targets this subunit both in vitro and ex vivo. Interestingly, this effect is observed in SPNs activated by optical stimulation of either cortical or thalamic glutamatergic afferents. We also found that treatment of striatal slices with antibodies targeting $\alpha$-syn prevents the $\alpha$-syn-induced loss of LTP and the reduced synaptic localization of GluN2A NMDA receptor subunit suggesting that this strategy might counteract synaptic dysfunction occurring in Parkinson's disease.
\end{abstract}

KEYWORDS: Dopamine; Glutamate; Long-term potentiation; Monoclonal antibodies; Oligomers; Optogenetics; Parkinson's disease; Synaptic plasticity; Striatum. 


\section{INTRODUCTION}

Alpha-synuclein ( $\alpha$-syn) is a 140 amino acids presynaptic protein causally involved in the pathogenesis of Parkinson's Disease (Spillantini and Goedert, 2016). The main mechanisms of $\alpha-$ syn-mediated cellular damage involve mitochondrial function, endoplasmic reticulum-Golgi trafficking, calcium ions influx through formation of pore-like structures, proteins degradation and oxidative stress (Gallegos et al., 2015; Wong and Krainc, 2017).

The protein exists as unstructured monomer widely distributed in the central nervous system and is involved in the regulation of presynaptic vesicle pool, neurotransmitter release, synaptic function and plasticity (Abeliovich et al., 2000; Cabin et al., 2002). However, $\alpha$-syn monomers have the intrinsic tendency to aggregate in structures of higher molecular weights (Gallegos et al., 2015) and in particular conditions, such as $\mathrm{pH}$ variations, oxidative stress, mutations and overexpression of $\alpha$ syn gene. This process can lead to the formation of $\alpha$-syn oligomers, protofibrils and eventually fibrils, the main components of Lewy Bodies (LBs) (Spillantini et al., 1997; Wong and Krainc, 2017). While LBs represent a neuropathological hallmark of Parkinson's disease and the histological sign of synucleinopathies, they show no actual direct neurotoxic properties (Tanaka et al., 2004). Mechanisms mediating $\alpha$-syn toxicity in humans have not been determined yet, however, several studies point to $\alpha$-syn oligomers and to protofibrils as major players in synaptic dysfunction (Diogenes et al., 2012; Rockenstein et al., 2014).

The striatum, a subcortical nucleus receiving major excitatory inputs from the cortex and the thalamus (Ding et al., 2008), is a brain region particularly involved in Parkinson's disease and oligomeric forms of $\alpha$-syn may affect synaptic transmission and long-term synaptic plasticity in this structure, leading to the motor and behavioural deficits observed in Parkinson's disease (Tozzi et al., 2016). 
Although in Parkinson's disease models pathological changes in synaptic plasticity have been widely reported in vivo as well as in vitro (Calabresi et al., 2014), at present, no data are available on the effects of $\alpha$-syn on synaptic transmission and synaptic plasticity of striatal spiny projection neurons (SPNs) and their possible underlying mechanisms.

SPNs are GABAergic output neurons, which represent the large majority of neuronal population in the striatum (Calabresi et al., 2014). They express a peculiar NMDA subunit receptor composition including GluN2A and GluN2B subunits (Dunah et al., 2000; Gardoni et al., 2006), whose distinct activation regulates glutamatergic inputs in striatal SPNs. In fact, the selective pharmacological modulation of these subunits differentially alters amplitude and kinetics of NMDARs responses also affecting responses to dopaminergic modulation (Jocoy et al., 2011; Vastagh et al., 2012). Moreover, an unbalanced GluN2A/GluN2B subunit ratio of the striatal synaptic NMDAR is a key element in the regulation of motor behaviour and synaptic plasticity in Parkinson's disease (Mellone et al., 2015).

For this reason, it is of great importance to assess whether $\alpha$-syn could alter synaptic transmission and plasticity in striatal SPNs by interfering with different NMDAR subunits.

In the present study, by utilizing a multidisciplinary approach including electrophysiological, optogenetic, immunofluorescence and molecular techniques as well as behavioural testing, we have determined the pathological effects of $\alpha$-syn on the striatal network controlled by NMDAR subunits.

Finally, we have provided the first proof of concept evidence that antibodies against $\alpha$-syn can prevent these pathological synaptic dysfunctions. 


\section{MATERIALS AND METHODS}

All experimental procedures were conducted in conformity with the European Directive 2010/63/EU, in accordance with protocols approved by the Animal Care and Use Committee at the University of Perugia (Perugia, Italy) and IRCCS Fondazione Santa Lucia (Rome, Italy).

\section{Animals}

For electrophysiological and behavioural experiments we used 2-3 month-old male Wistar rats (Charles River, Calco, Italy); 2 month-old transgenic male BAC Drd1a-tdTomato mice containing a mouse Drdla promoter directing the expression of a modified DsRed fluorescent protein (B6.CgTg(Drd1a-tdTomato)6Calak/J, tdTomato, The Jackson Laboratory, Bar Harbor, Maine, U.S.A.) and B6.Cg-Tg(Thy1-COP4/EYFP)18Gfng/J transgenic mice (Thy1-ChR2-YFP) expressing the lightactivated ion channel Channelrhodopsin-2 (ChR2) fused to yellow fluorescent protein under the control of the mouse thymus cell antigen 1 (Thy1) promoter. Selective expression of ChR2 has been demonstrated both in cortical neurons of layer V and thalamic neurons (Arenkiel et al., 2007; Lim et al., 2012).

Animals were housed at $23^{\circ} \mathrm{C}$ room temperature with food and water ad libitum and a 12-hour light-dark cycle. All efforts were made to minimize the number of animals used and their suffering.

\section{Electrophysiology}

Coronal brain slices including the cortex and the striatum were cut from Wistar rats and tdTomato mice using a vibratome at $220-240 \mu \mathrm{m}$ thickness. To better preserve the connectivity of thalamic fibres to the striatum, para-horizontal slices $(250 \mu \mathrm{m})$ were cut from Thy1-ChR2-YFP mice for optogenetic experiments (Smeal et al., 2007; Ding et al., 2008; Sciamanna et al., 2012). Slices were maintained in a Kreb's solution, bubbled with a $95 \% \mathrm{O}_{2}-5 \% \mathrm{CO}_{2}$ gas mixture at room temperature containing (in mM): $126 \mathrm{NaCl}, 2.5 \mathrm{KCl}, 1.2 \mathrm{MgCl}_{2}, 1.2 \mathrm{NaH}_{2} \mathrm{PO}_{4}, 2.4 \mathrm{CaCl}_{2}, 10$ glucose, and 25 $\mathrm{NaHCO}_{3}$ (Calabresi et al., 1992b). Single slices were transferred to a recording chamber and submerged in a continuously flowing Krebs' $\left(34{ }^{\circ} \mathrm{C} ; 2.5-3 \mathrm{ml} /\right.$ minute) bubbled with a $95 \% \mathrm{O}_{2}-5 \%$ 
$\mathrm{CO}_{2}$ gas mixture. The internal solution for path-clamp recordings contained (in $\mathrm{mM}$ ): $145 \mathrm{~K}^{+}-$ gluconate, $0.1 \mathrm{CaCl}_{2}, 2 \mathrm{MgCl}_{2}, 0.1$ EGTA, $10 \mathrm{HEPES}, 0.3 \mathrm{Na}-\mathrm{GTP}$ and $2 \mathrm{Mg}$-ATP, adjusted to $\mathrm{pH}$ 7.3 with $\mathrm{KOH}$. For recording NMDAR-mediated currents, neurons were held at $+40 \mathrm{mV}$ membrane holding potential and the internal solution contained (in $\mathrm{mM}$ ): $120 \mathrm{CsMeSO}_{3}, 10 \mathrm{CsCl}, 8 \mathrm{NaCl}, 2$ $\mathrm{MgCl}_{2}, 10$ HEPES, 0.2 EGTA, 10 TEA, 5 QX314, 0.3 Na-GTP and $2 \mathrm{Mg}$-ATP, adjusted to pH 7.3 with $\mathrm{CsOH}$. Only neurons identified as striatal SPNs were considered for the experiments. Normally, for each animal we obtained 2-3 experiments. SPNs were identified by their hyperpolarized resting membrane potential (RMP, $\sim-80 \mathrm{mV}$ ), absence of spontaneous action potential discharge and presence of tonic firing activity during current-induced membrane depolarization. Signals were amplified with a Multiclamp 700B amplifier (Molecular Devices), recorded and stored on PC using pClamp10. Whole-cell voltage-clamp recordings (access resistance 15-30 M $\Omega$; holding potential $-80 \mathrm{mV}$ ) were performed with borosilicate pipettes (4-7 M 2$)$ filled with the standard internal solution. A bipolar electrode, connected to a stimulation unit (Grass Telefactor), was located in the white matter between the cortex and the striatum to stimulate glutamatergic fibres $(0.1 \mathrm{~Hz})$ and evoke excitatory post-synaptic currents (EPSCs), while the recording electrode was placed in the dorsolateral striatum. In all patch-clamp experiments $50 \mu \mathrm{M}$ picrotoxin was added to the external medium to block $\mathrm{GABA}_{\mathrm{A}}$ receptors. To induce LTP or longterm depression (LTD), a high-frequency stimulation (HFS) protocol consisting of three trains of 3 seconds (20 seconds interval) was delivered at $100 \mathrm{~Hz}$. During HFS protocol, the stimulus intensity was increased to supra-threshold levels. External $\mathrm{Mg}^{2+}$ ions were omitted to maximize the contribution of NMDARs during LTP experiments (Calabresi et al., 1992b). The AMPA receptor (AMPAR)- and NMDAR-mediated synaptic currents (AMPA-EPSCs and NMDA-EPSCs) were recorded in pharmacological isolation, with $50 \mu \mathrm{M}$ picrotoxin plus $50 \mu \mathrm{M}$ APV and $50 \mu \mathrm{M}$ picrotoxin plus $10 \mu \mathrm{M} C N Q X$, respectively. AMPAR rectification index (RI) was obtained by dividing the AMPA-EPSC amplitude measured at $+40 \mathrm{mV}$ by the one measured at $-80 \mathrm{mV}$. 
NMDA/AMPA ratios were calculated by dividing the NMDA-EPSC positive peak amplitude, acquired at $+40 \mathrm{mM}$ holding potential in the presence of $50 \mu \mathrm{M}$ picrotoxin plus $10 \mu \mathrm{M}$ CNQX, by the EPSC negative peak amplitude acquired at $-80 \mathrm{mV}$ in picrotoxin.

\section{Optogenetic}

Optical stimulation of cortex or thalamus was performed using an infinity cube (CAIRN Research, UK), consisting of a $470 \mathrm{~nm}$ filter system mounted on an Olympus BX-51WI microscope and controlled by an Optoflash (CAIRN Research, UK) LED light Switch. The experiments were performed on Thy1-ChR2-YFP mice. Light power at microscope objective exit was 2-30 $\mathrm{mW} / \mathrm{mm}^{2}$. Spotlight was made by utilizing a diaphragm placed along the light path. Slices were illuminated from the top and a round spot was created with a radius of approximately 500-600 $\mu \mathrm{m}$ in order to avoid light spread to unwanted brain structures and delivered on the cortex or the thalamus. A 3 mseconds light pulse was delivered every 15 seconds on cortical layer $\mathrm{V}$ or in the thalamus in order to evoke EPSCs in MSNs.

\section{Preparation of alpha-synuclein in Krebs solution}

Different concentrations of $\alpha$-syn cocktail (3, 10, 30 and $300 \mathrm{nM}$ ) (referred in the text as $\alpha$-syn OLIGO) were prepared in Krebs solution bubbled with a $95 \% \mathrm{O}_{2}-5 \% \mathrm{CO}_{2}$ gas mixture at room. The solutions were incubated up to 1 hour taking aliquots at different time points.

\section{Alpha-synuclein preformed fibrils (PFF) preparation}

Lyophilized fresh monomeric $\alpha$-syn $(0.33 \mathrm{mg}$ ) (recombinant human $\alpha$-syn) was dissolved in sterile PBS to a final concentration of $1 \mathrm{mg} / \mathrm{mL}(\approx 70 \mu \mathrm{M}) .300 \mu \mathrm{L} \alpha$-syn-containing buffer has been incubated for 7 consecutive days at $37{ }^{\circ} \mathrm{C}$ on a shaker $(300 \mathrm{rpm})$ in a sterile 96 -well plate. At the end of this procedure the $\alpha$-syn PFF aliquots were stored at $-80{ }^{\circ} \mathrm{C}$ until the use for intra-brain injection. 


\section{Transmission electron microscopy (TEM)}

The samples $(5 \mu \mathrm{l})$ were deposited on a glow-discharged Formvar-Carbon 300-mesh coated copper grid (Agar Scientific, UK) for 2 minutes, blotted with Whatman filter paper and negatively stained with $2 \%$ uranyl acetate for few seconds. Grids were examined with an Energy Filtering transmission electron microscope Leo 912 ab (Zeiss, Germany) operating at 100KV and digital images were acquired by a CCD camera (Proscan, Germany) $1 \mathrm{kx} 1 \mathrm{k}$ with the iTEM software (Olympus, Germany).

\section{Detection of alpha-synuclein oligomers in the Krebs solution}

A 384-well ELISA microplate (Nunc MaxiSorb, Nunc) was coated by overnight incubation at $4{ }^{\circ} \mathrm{C}$ with SynO2 $(0.2 \mu \mathrm{g} / \mathrm{ml})$ in $200 \mathrm{mM}$ NaHCO3, pH $9.6(50 \mu \mathrm{l} /$ well $)$. The plate was then washed with PBST and incubated with $100 \mu \mathrm{l} /$ well of blocking buffer for 2 -hours at $37{ }^{\circ} \mathrm{C}$. After washing, $50 \mu \mathrm{l}$ of the samples $(1 \mathrm{nM})$ was added to each well, and plates were incubated at $37{ }^{\circ} \mathrm{C}$ for 2.5 -hours. FL-140 (rabbit polyclonal antibody, Santa Cruz Biotechnology, Santa Cruz, CA, USA), diluted in blocking buffer at 1:1 K, was added to the appropriate wells, and incubated at $37{ }^{\circ} \mathrm{C}$ for $2 \mathrm{~h}$. Next, the plate was washed and incubated for $2 \mathrm{~h}$ at $37{ }^{\circ} \mathrm{C}$ with $50 \mu \mathrm{l} /$ well of goat anti-rabbit IgG HRP (Jackson ImmunoResearch) diluted in blocking buffer $(1: 15 \mathrm{~K})$. After washing, the plate was incubated with $50 \mu \mathrm{l} /$ well of an enhanced chemiluminescent substrate (SuperSignal ELISA Femto, Pierce Biotechnology, Rockford, IL). The chemiluminescence, expressed in relative light units, was immediately measured using VICTOR ${ }^{\mathrm{TM}}$ X3 multilabel plate reader (PerkinElmer).

\section{Thioflavin $\mathbf{T}$ binding assay}

The formation of $\alpha$-syn oligomers was confirmed by Thioflavin T (ThT) binding assay (Nilsson, 2004). ThT was incubated at a final concentration of $20 \mu \mathrm{M}$ in different $\alpha$-syn solutions (30 $\mathrm{nM}$ and $300 \mathrm{nM}$, respectively) collected at T0 and after 60 minutes of incubation in Krebs solution bubbled with a $95 \% \mathrm{O}_{2}-5 \% \mathrm{CO}_{2}$ gas mixture at room temperature. Emission wavelength scan was performed 
with an excitation wavelength of $450 \mathrm{~nm}$ using a plate reader (BMG Labtech Clariostar). The assay was performed in triplicate.

\section{Immunoprecipitation of alpha-synuclein oligomers formed in Krebs solution}

$500 \mu \mathrm{l}$ of the samples collected at time points 0 and 30 minutes were incubated with 0.043 and 4.3 $\mathrm{X} 10^{-5} \mu \mathrm{g} / \mathrm{ml}$ of either SynO2 or Syn211 antibodies for 2-hours at room temperature. $100 \mu \mathrm{l}$ of protein $\mathrm{G}$ beads was added and incubated for 1-hour at room temperature. The samples were centrifuged and the supernatant were collected and checked for the presence of $\alpha$-syn oligomers by ELISA. The bound $\alpha$-syn oligomers form the beads were eluted by adding $50 \mu 1$ of $0.2 \mathrm{M}$ glycine $\mathrm{pH} 2.5$ and immediately neutralized with $5 \mu \mathrm{l}$ of $1 \mathrm{M}$ Tris $\mathrm{pH} 8.0$ and taken for western blot analysis.

\section{Subcellular fractionation}

For the extraction of triton-insoluble postsynaptic fraction (TIF), striatal areas were carefully isolated and collected on dry ice from control slices and slices treated with $\alpha$-syn alone $(30 \mathrm{nM})$ and with Abs against $\alpha$-syn (Syn211 and SynO2). Striatal areas were then homogenized with a Teflonglass potter in ice-cold buffer containing (in mM) 320 sucrose, 1 Hepes, $1 \mathrm{MgCl}_{2}, 1 \mathrm{NaHCO}_{3}, 0.1$ phenylmethylsulfonylfluoride at $\mathrm{pH} 7.4$ in the presence of Complete ${ }^{\mathrm{TM}}$ Protease Inhibitor Cocktail Tablets (Roche Diagnostics, Basel, Switzerland) and phosSTOPTM Phosphatase Inhibitor (Roche Diagnostics). The sample was spun at $13.000 \mathrm{~g} \mathrm{x} 15$ minutes at $4{ }^{\circ} \mathrm{C}$. The resulting pellet was resuspended in Triton- $\mathrm{KCl}$ buffer $(0.5 \%$ Triton $\mathrm{X}-100$ and $150 \mathrm{mM} \mathrm{KCl})$ and, after 15 minutes incubation on ice, it was spun further at $100.000 \mathrm{~g} \mathrm{x} \mathrm{1-hour} \mathrm{at} 4{ }^{\circ} \mathrm{C}$. The pellet (triton-insoluble postsynaptic fraction, TIF) was resuspended in $20 \mathrm{mM}$ Hepes buffer supplemented with Complete ${ }^{\mathrm{TM}}$ Protease Inhibitor Cocktail Tablets and stored at $-80{ }^{\circ} \mathrm{C}$.

\section{Western blotting (WB)}

The levels of NMDARs and AMPARs in the TIF were analysed by WB. TIF samples were separated onto a $7 \%$ acrylamide/bis acrylamide gel. Proteins were blotted onto a nitrocellulose 
membrane (Bio-Rad, Hercules, CA, USA) and probed with the appropriate primary antibodies followed by the corresponding HRP-conjugated secondary antibodies. Labeling detection was performed with ChemiDoc MP Imaging System (Bio-Rad) and images were acquired with ImageLab software (Bio-Rad). The primary antibodies used in this study are anti-GluN2A 1:500 (Sigma-Aldrich, St. Louis, MO, USA), anti-GluN2B 1:500 (Neuromab, Davis, CA, USA), antiGluA1 1:1000 (Neuromab), anti-GluA2 1:500 (Neuromab) and anti-tubulin 1:20000 (SigmaAldrich). The latter was always used as loading control for normalization.

\section{Immunolabeling}

Primary antibodies of hu- $\alpha$-Syn (1:1000, sc-12767, Santa Cruz Biotechnology) in combination with hu- $\alpha$-Syn phosphorylated on Ser129 (hu- $\alpha$-Syn-phospho S129, ab51253; abcam) or in combination with tyrosine hydroxylase (TH 1:500, AB152; Merck Millipore) were used. $30 \mu \mathrm{m}$ coronal slices, obtained with the use of a vibratome (Leica VT1000 S) were incubated overnight at $4{ }^{\circ} \mathrm{C}$ with a PBS solution containing BSA $0.1 \%$ and the combination of two primary antibodies. After rinsing in PBS, the sections were incubated for 2-hours with a mixture of secondary antibodies: goat antirabbit Alexa Fluor 647, goat anti-mouse Alexa Fluor 488 (1:300; AP187SA6 and AP124JA, Merck Millipore). After subsequent washes in PBS, brain slices were incubated with DAPI (S7113; Merck Millipore) for 10 minutes and finally mounted on gelatin-coated slides and coverslipped. The specificity of immunoreaction was controlled by omitting primary antibodies. Images of the medial and lateral striatum (60x magnification) were taken using a fully motorized confocal microscope Nikon A1 and Nikon NIS Elements Advanced Research software.

\section{Surgery}

A total of 30 male Wistar rats (Charles River, Italy) were housed three x cage with free access to chow and water under a 12:12 h light-dark cycle. All surgical operations were performed under ketamine/xylazine anaesthesia. Animals received two bilateral intrastriatal injections of either $\alpha$-syn PFF $(n=9)$, OLIGO $(n=6)$ or PBS (control, $n=15)$. The volume injected using a $10 \mu$ Hamilton 
microsyringe was $1 \mu \mathrm{l}$ for each site. The injection rate was $0.38 \mu \mathrm{l} /$ minute and for each site the cannula was left in place for additional $2 \mathrm{~min}$ before retracting it. The coordinates, based on a previous study using these parameters to perform striatal lesions (Kirik et al., 1998), were the following: 1) anteroposterior (AP) +1.0; mediolateral (ML) +3.0; dorsoventral (DV) -5.0; 2) AP +1.0; $\mathrm{ML}$-3.0; DV -5.0. ML coordinates were taken from lambda, AP coordinates were from bregma, according to Paxinos and Watson (Paxinos, 2005). Tooth bar was set at 0.00 . After the surgery the animals were monitored until awake and then brought back to animal facility. Six weeks after the surgery the animals were tested for motor behaviour and cognition deficit.

\section{Visuo-Spatial Memory Task}

Visuo-Spatial Memory Task was a rat adapted version of the visuo-spatial memory task previously described in mouse (De Leonibus et al., 2007; De Leonibus et al., 2009; Rodo et al., 2017). The test was divided in 10 sessions of 5 minute each. The interval between the sessions was 3 minutes. Briefly, during session 1 (S1), rats were placed in an empty a square open field to habituate to the context $(60 \times 60 \mathrm{~cm})$. After this, from session 2 to session $7(\mathrm{~S} 2-\mathrm{S} 7)$, rats were free to explore three objects positioned in the arena to allow them to learn information about the objects and their spatial location in the arena (habituation phase). To test whether the animals learned spatial information related to the position of the objects, during the spatial test session 8 (S8), the objects configuration was changed by moving one object (the displaced object, DO) and leaving the other two objects in the same position (non-displaced objects, NDO1 and NDO2). In session 9 (S9), the configuration of the objects was kept unchanged to let the animal to habituate to the spatial change. In the last session (S10), to test whether animals learned information about the shape of the object, one of the familiar objects was replaced by a new one (substituted object, SO); the other two objects were left unchanged (non-substituted objects, NSO1 and NSO2). Object exploration was defined as the time in which the nose of the animal was in directed toward the object ( $<2 \mathrm{~cm}$ distance). The animals' ability to selectively react to the spatial change was analysed by calculating the spatial exploration 
index $(\mathrm{DO}=\mathrm{DO}[\mathrm{S} 8]-\mathrm{DO}[\mathrm{S} 7] ; \mathrm{NDO}=\mathrm{NDO}[\mathrm{S} 8]-\mathrm{NDO}[\mathrm{S} 7])$, while the animals' ability to selectively react to the non-spatial change (novel object) was analysed by calculating non-spatial exploration index $(\mathrm{SO}=\mathrm{SO}[\mathrm{S} 10]-\mathrm{SO}[\mathrm{S} 9] ; \mathrm{NSO}=\mathrm{NSO}[\mathrm{S} 10]-\mathrm{NSO}$ [S9]). Basal motor activity was evaluated during $\mathrm{S}$, by measuring time interval changes in distance travelled $(\mathrm{cm})$, immobility time (seconds) and maximal speed (cm/second).

\section{Statistical Analysis}

Data analysis of electrophysiological experiments was performed off-line using Clampfit10 (Molecular Devices) and GraphPad Prism 5.0 (GraphPad Software). Values in the text and figures are mean \pm standard error of the mean (SEM), $n$ representing the number of recorded neurons. Changes of EPSC amplitude induced by drugs or by stimulation protocols were expressed as a percentage of the baseline, the latter representing the normalized EPSC mean amplitude acquired during a stable period (10-15 minutes) before delivering drugs or stimulation and statistically evaluated by the Student's $t$-test for unpaired samples. LTP and LTD presence were statistically verified with the Student's $t$-test for paired samples by comparing in each experiment values of the EPSC amplitudes at 25-30 minutes after the application of the HFS protocol relatively to the baseline. Current-voltage (I-V) curves were compared using the two-way ANOVA. The significance level was established at $\mathrm{P}<0.05$. For WB experiments, significance of the differences was determined by one-way ANOVA followed by Tukey's post-hoc test or by unpaired Student's $t$ test as appropriate. For ThT binding assay significance of the differences was determined by Student's $t$-test. For behavioural analysis, data were analysed by using two-way Analysis of Variance (ANOVA) for repeated measure, with treatment (two levels: control and $\alpha$-syn) as between factor. Duncan post hoc analysis was used when appropriate.

When parametric tests were used, normality was checked with Shapiro-Wilk test and visual inspection of data distribution. The criterion for significance was set at $\mathrm{P}<0.05$. We used Shapiro- 
Wilk test for its level of power compared to other procedures in small sample size scenarios (Royston, 1993).

\section{Drugs}

Picrotoxin, D-(-)2-amino-5-phosphonopentanoic acid (APV), 6-cyano-7- nitroquinoxaline-2,3dione disodium (CNQX), threo ifenprodil hemitartrate, 3-chloro-4-fluoro-N-[4-[[2(phenylcarbonyl)hydrazino]carbonyl]benzyl]benzenesulfonamide (TCN-201), NVP-AAM077 were from Tocris; dopamine hydrochloride (DA) was from Sigma.

Drugs were bath-applied by switching the Kreb's solution to one containing known concentrations of drugs. Drugs applied in the recording chamber were delivered for at least 10 minutes before recording AMPA-EPSC and NMDA-EPSC and maintained throughout the experiment. We used two different mouse antibodies against $\alpha$-syn: a monoclonal IgG1 Ab, raised against the amino acid 121-125 of human $\alpha$-syn, which recognizes all forms of the protein (Syn211, Santa Cruz Biotechnology, Inc.,) (El-Agnaf et al., 2003) and a monoclonal IgG1 Ab which has a higher affinity to oligomers more than $\alpha$-syn fibrils (SynO2) (Vaikath et al., 2015). Abs and $\alpha$-syn were provided by Prof. O. El-Agnaf (U.A.E. University, United Arab Emirates). Corticostriatal slices were incubated in a solution containing $8.6 \mu \mathrm{g} \alpha$-syn, corresponding to a dose of $30 \mathrm{nM}$, plus the $\mathrm{Ab}$ for 1 hour $(1: 1, \mu \mathrm{g} / \mu \mathrm{g})$. The Syn211 Ab was further diluted 1:10 $(0.043 \mu \mathrm{g} / \mathrm{ml})$ and 1:100 $(0.0043$ $\mu \mathrm{g} / \mathrm{ml})$, while SynO2 Ab was used at dilution 1:10.000 $\left(4.3 \times 10^{-5} \mu \mathrm{g} / \mathrm{ml}\right)$.

\section{RESULTS}

Alpha-synuclein does not affect basal membrane properties and LTD but impairs LTP in striatal SPNs

In order to characterize the $\alpha$-syn effect on striatal SPNs we first explored the effect of this protein on the basal membrane properties performing patch-clamp recordings of SPNs in striatal slices obtained from 2-3 months-old rats. The current-voltage relationship was evaluated applying 
hyperpolarizing and depolarizing steps of currents to SPNs of control slices and of slices incubated for 1-hour with $30 \mathrm{nM}$ monomeric/oligomeric $\alpha$-syn freshly diluted in the Kreb's solution (see Materials and Methods). We found that the measured electrical properties were unaffected by $\alpha$-syn since statistical analysis showed no significant differences in the I-V curves between SPNs recorded in control slices $(\mathrm{n}=5)$ and in slices treated with $\alpha$-syn $(\mathrm{n}=4)$ (control $v s \alpha$-syn, $\mathrm{P}>0.05)$ (Fig. 1A). Striatal slices were subsequently tested to assess the effect of $30 \mathrm{nM} \alpha$-syn on long-term changes of synaptic transmission (LTD and LTP) of SPNs. EPSCs were recorded for 10 minutes to obtain a stable baseline and then for 30 minutes after the application of the HFS protocol. We found that SPNs recorded in control and in $\alpha$-syn-incubated slices showed LTD of similar amplitudes (Calabresi et al., 1992a). In fact, the EPSCs amplitude was reduced by $46.0 \pm 2.8 \%(\mathrm{n}=6)$ and by $49.8 \pm 2.1 \%(n=6)$ in SPNs from control slices and $\alpha$-syn-incubated slices, respectively $\left(\mathrm{t}_{(10)}=1.094\right.$, $\mathrm{P}<0.05$, pre $v s 30 \mathrm{~min}$ after HFS, Fig. 1B). To explore the effect of $\alpha$-syn on SPNs LTP, EPSCs were recorded before and for 30 minutes after the application of magnesium free HFS protocol. While SPNs from control slices showed a robust LTP of $148.7 \pm 3.2 \%\left(n=7, t_{(6)}=15.37, P<0.001\right)$, neurons from $\alpha$-syn-treated slices showed no LTP ( $\mathrm{n}=7, \mathrm{P}>0.05$, pre $v s$ 30min after HFS, Fig. 1C). We also analysed the EPSC amplitude, measured $30 \mathrm{~min}$ after the induction of the LTP, in the presence of different concentrations of $\alpha$-syn. We found that while $3 \mathrm{nM} \alpha$-syn (n=6) did not affect this parameter, both $10 \mathrm{nM}(\mathrm{n}=7)$ and $30 \mathrm{nM}(\mathrm{n}=7)$ produced a significant reduction. Higher concentrations of $\alpha$-syn (300 nM, n=6) did not further decrease the EPSC amplitude (Fig. 1D). It has been postulated a major detrimental effect of $\alpha$-syn is the reduction of striatal dopamine (DA) levels (Ghiglieri et al., 2018), thus the $\alpha$-syn-induced LTP impairment could be induced by lower DA levels in the synaptic cleft. To test this hypothesis, $30 \mu \mathrm{M}$ exogenous DA were bath applied before the HFS in order to obtain a recovery of the LTP in the presence of $\alpha$-syn $(n=6)$. Surprisingly, DA failed to restore this form of synaptic plasticity suggesting that other nondopaminergic mechanisms might be involved in the loss of LTP in the presence of $\alpha$-syn (Fig. 1E). 
In order to investigate the time- and dose-dependent formation of $\alpha$-syn oligomers in the Krebs solution used for the electrophysiological experiments, $\alpha$-syn samples at time 0 and 30 min were taken and measured by specific oligomeric ELISA. As expected, the formation of $\alpha$-syn oligomers was time- and dose-dependent (Fig. $1 \mathrm{~F}, * \mathrm{P}<0.05, * * \mathrm{P}<0.01$ ), as higher level of $\alpha$-syn oligomers were detected in the $300 \mathrm{nM} \alpha$-syn samples collected at $30 \mathrm{~min}$. To evaluate the presence of fibrils we used the Thioflavin T (ThT) fluorescence assay; the results clearly show that little fluorescence was detected with the $30 \mathrm{nM}$ concentration, independently on the timing of sampling (Fig. 1G, $* * * \mathrm{P}<0.001)$

\section{Alpha-synuclein affects SPNs glutamatergic transmission by selectively targeting the GluN2A- NMDAR current, but not the GluN2B-NMDAR and AMPAR currents}

We then evaluated the effect of $\alpha$-syn on the AMPA- and NMDA-EPSCs since both these glutamate receptors exert a pivotal role in the long-term changes of synaptic plasticity of SPNs. To isolate AMPA-EPSCs, SPNs were recorded in the presence of $50 \mu \mathrm{M}$ of the NMDAR antagonist APV and $50 \mu \mathrm{M}$ of the $\mathrm{GABA}_{\mathrm{A}} \mathrm{R}$ antagonist picrotoxin. After obtaining stable EPSCs for 10 minutes, synaptic currents were measured in the presence of $30 \mathrm{nM} \alpha$-syn for additional 10 minutes. We found no significant difference between AMPA-EPSCs recorded before and following $\alpha$-syn application ( $\mathrm{n}=6, \mathrm{P}>0.05$, Fig. 2A). We also evaluated the AMPAR rectification index (RI) calculated in control conditions and in the presence of $30 \mathrm{nM} \alpha$-syn (Fig. 2B). Statistical analysis shows that in these conditions the AMPA RI was not significantly different from that measured before $\alpha$-syn application ( $n=6, P>0.05$, Fig. $2 B$ ), further suggesting that in SPNs $\alpha$-syn does not alter the function of AMPAR.

We also explored whether $\alpha$-syn affected the NMDAR by targeting the GluN2A- or the GluN2Bmediated NMDA-EPSC (GluN2A-EPSC and GluN2B-EPSC). To isolate the GluN2A-EPSC, synaptic responses were recorded in the presence of $10 \mu \mathrm{M}$ of the AMPAR antagonist CNQX, 50 
$\mu \mathrm{M}$ picrotoxin and $3 \mu \mathrm{M}$ of the GluN2B antagonist ifenprodil. We found that after recording a stable baseline for 10 minutes, the subsequent application of $30 \mathrm{nM} \alpha$-syn significantly reduced the GluN2A-EPSC by $40.8 \pm 6.1 \%\left(\mathrm{n}=6, \mathrm{t}_{(5)}=6.684, \mathrm{P}<0.05\right.$, Fig. $\left.2 \mathrm{C}\right)$. We subsequently isolated the GluN2B-EPSC by recording synaptic responses in the presence of CNQX, $50 \mathrm{nM}$ of the GluN2A antagonist NVP-AAM077 and picrotoxin. The application of $30 \mathrm{nM} \alpha$-syn did not affect the GluN2B-EPSC ( $n=6, P>0.05$, Fig. 2D). Similarly, we found that the GluN2B-EPSC, isolated using the more specific GluN2A antagonist TCN-201 $(10 \mu \mathrm{M})$, was not significantly affected by $\alpha$-syn application ( $n=6, P>0.05$, Fig. 2E). Taken together these findings show that $\alpha$-syn affects the SPNs NMDA-EPSC selectively targeting the GluN2A-containing NMDA receptors. Moreover, we also evaluated, by WB analysis in a triton-insoluble post-synaptic fraction (TIF), the possibility that alterations in the molecular composition of ionotropic glutamate receptors may lead to the observed synaptic alterations in SPNs induced by $\alpha$-syn. In line with the electrophysiological experiments, we found that incubation (1-hour) of the slices in $30 \mathrm{nM} \alpha$-syn did not alter GluA1 and GluA2 AMPAR subunit synaptic levels ( $\mathrm{n}=8, \mathrm{P}>0.05, \alpha$-syn $v$ s control, Fig. $2 \mathrm{~F}$ ) while it significantly reduced the GluN2A but not GluN2B NMDAR subunit synaptic levels ( $\mathrm{n}=6$, GluN2A, $\mathrm{P}=0.014$, $\alpha$-syn vs control, Fig. 2G).

\section{GluN2A but not GluN2B NMDAR subunit plays a critical role in the induction of LTP in} SPNs

To ascertain the involvement of GluN2A and GluN2B NMDAR subunits in LTP, we first induced this form of synaptic plasticity in the presence of $3 \mu \mathrm{M}$ of the GluN2B antagonist ifenprodil, to isolate the GluN2A-EPSC. In these conditions we found a normal LTP since the increase of EPSCs amplitude obtained after the delivery of HFS protocol was not different from that one of control slices (control, $147.2 \pm 3.0 \%, \mathrm{n}=6$; ifenprodil, $143.3 \pm 2.8 \%, \mathrm{n}=5, \mathrm{P}>0.05$, Supplementary Fig. 1). Conversely, the application of the GluN2A antagonists NVP-AAM077 (50 nM, n=5) or TCN-201 
(10 $\mu \mathrm{M}, \mathrm{n}=5)$ completely prevented the LTP induction $(\mathrm{P}>0.05$, Supplementary Fig. 1), further confirming that the NMDAR expressing the GluN2A subunit mediates the LTP induction of striatal SPNs.

\section{Alpha-synuclein affects LTP and NMDAR-mediated synaptic currents in SPNs of both direct and indirect pathways}

To investigate whether SPNs expressing either the D1-like or D2-like DA receptors (Calabresi et al., 2014) were differentially affected by $\alpha$-syn, we recorded SPNs from striatal slices of dtTomato mice. In these animals, we firstly optically identified fluorescent (D1-like expressing: D1Rpositive) and non-fluorescent (putative D2-like expressing: putative D2R-positive) SPNs (Fig. 3A). Subsequently, we patch-clamped SPNs for an electrophysiological characterization and for LTP analysis in both these neuronal sub-populations. We found that both fluorescent (D1R-positive) and non-fluorescent (putative D2R-positive) SPNs showed LTP of similar amplitude. In fact, 30 minutes after the induction of the HFS protocol, the EPSC amplitude was increased respect to the baseline by $134.6 \pm 4.0 \%\left(\mathrm{n}=6, \mathrm{t}_{(5)}=11.33, \mathrm{P}<0.001\right)$ and $137.1 \pm 5.9 \%\left(\mathrm{n}=6, \mathrm{t}_{(5)}=5.452, \mathrm{P}<0.001\right)$, respectively in fluorescent and non-fluorescent SPNs (Fig. 3B).

Moreover, we aimed at verifying whether $\alpha$-syn equally affected LTP of SPNs of both neuronal sub-populations. Striatal slices from dtTomato mice were incubated for 1-hour with $30 \mathrm{nM} \alpha$-syn. In these conditions we found that the induction of LTP was completely prevented in both fluorescent ( $\mathrm{n}=5, \mathrm{P}>0.05)$ and non-fluorescent SPNs (n=5, P>0.05) (Fig. 3C-D).

Since $\alpha$-syn affected NMDA-EPSCs (Fig. 2C-E), we tested whether this glutamatergic current was differentially altered by $\alpha$-syn in SPNs expressing and not expressing (putative D2R-positive) the D1 DA receptor. The NMDA-EPSC amplitudes were recorded in pharmacological isolation in the presence of $50 \mu \mathrm{M}$ picrotoxin plus $10 \mu \mathrm{M}$ CNQX in both fluorescent and non-fluorescent SPNs of dtTomato mice. After acquiring a stable baseline for 10 minutes, $30 \mathrm{nM} \alpha$-syn was bath applied for 
subsequent 10 minutes. We found that $\alpha$-syn significantly reduced the NMDA-EPSC in both SPN populations. The EPSCs were reduced by $27.2 \pm 4.2 \%$ in D1R-positive SPNs $\left(n=6, t_{(5)}=6.510\right.$, $\mathrm{P}<0.05$, Fig. 3E) and by $30.1 \pm 6.3 \%$ in putative D2R-positive SPNs $\left(\mathrm{n}=4, \mathrm{t}_{(3)}=4.764, \mathrm{P}<0.05\right.$, Fig. $3 \mathrm{~F})$ and the amplitude of these reductions was similar in these two groups of SPNs $(\mathrm{P}>0.05)$. These findings suggest that $\alpha$-syn exerts similar effects in SPNs of the direct and indirect basal ganglia pathways equally affecting the NMDAR-mediated synaptic current.

\section{Alpha-synuclein affects the NMDAR-current evoked in SPNs by cortico-striatal and thalamo- striatal optical stimulation}

Our data suggest that $\alpha$-syn reduced the GluN2A-EPSC amplitude following the stimulation of glutamatergic afferents to the striatum. However, striatal SPNs receive glutamatergic inputs by both the cortex and the thalamus and these pathways might be differentially regulated by pharmacological agents (Wu et al., 2015). Thus, we investigated whether $\alpha$-syn affected the NMDAR-mediated transmission in cortico-striatal synapses as well as in the thalamo-striatal pathway by using an optogenetic approach to better dissect inputs from these two brain structures. We firstly recorded NMDA-EPSCs in pharmacological isolation to evaluate the contribution of the GluN2A and GluN2B NMDAR subunits in cortico-striatal and thalamo-striatal synapses and then analysed the effect of $\alpha$-syn on these currents (Fig. 4A-H). SPNs EPSCs, evoked by optical stimulation of cortico-striatal projections, were recorded from Thy1-ChR2-YFP mice. GluN2A- or GluN2B-EPSCs were isolated in the presence of $50 \mu \mathrm{M}$ picrotoxin, $10 \mu \mathrm{M}$ CNQX and $3 \mu \mathrm{M}$ ifenprodil (GluN2B antagonist) or $10 \mu \mathrm{M}$ TCN-201 (GluN2A antagonist), respectively.

We found that the NMDA-EPSC was significantly reduced by $45.46 \pm 3.29 \%$ in the presence of the solution containing ifenprodil and by $35.04 \pm 5.47 \%$ in that with TCN-201 (ifenprodil, $\mathrm{n}=6$, $\mathrm{t}_{(5)}=13.86, \mathrm{P}<0.0001 ; \mathrm{TCN}-201, \mathrm{n}=6, \mathrm{t}_{(5)}=6.405, \mathrm{P}<0.05$, Fig. 4C). We subsequently tested the effect of $\alpha$-syn on the isolated GluN2A- and GluN2B-EPSC. $30 \mathrm{nM} \alpha$-syn significantly reduced the 
GluN2A-EPSC by $40.82 \pm 4.46 \%\left(\mathrm{n}=6, \mathrm{t}_{(5)}=9.148, \mathrm{P}<0.05\right.$, Fig. 4D). This effect was observed at 5 , but not at 2 minutes, after the $\alpha$-syn application (at least $n=4$ for all the time points, $P<0.001$, Fig. 4D). Notably, longer $\alpha$-syn applications (10 minutes) did not further decrease the NMDA current. Interestingly, we found no effect of $\alpha$-syn on the GluN2B-EPSC at all time-points tested (at least $\mathrm{n}=4$ for all time points, $\mathrm{P}>0.05$, Fig. 4D).

Striatal SPNs EPSCs were also evoked by optical stimulation of the thalamus of Thy1-ChR2-YFP mice to evaluate the contribution of the GluN2A and GluN2B currents in the NMDAR response activated by thalamic glutamatergic fibres. NMDA-EPSCs, recorded in the presence of picrotoxin plus CNQX, were significantly reduced by $34.08 \pm 2.87 \%$ in the presence of ifenprodil ( $\mathrm{n}=5$, $\mathrm{t}_{(4)}=11.87, \mathrm{P}<0.05$, Fig. $\left.4 \mathrm{~F}\right)$ and by $32.98 \pm 3.12 \%$ in TCN-201 $\left(\mathrm{n}=6, \mathrm{t}_{(5)}=10.57, \mathrm{P}<0.05\right.$, Fig. 4F $)$.

Isolated GluN2A and GluN2B currents were subsequently measured in the presence of $\alpha$-syn. We found that in these conditions the GluN2A-EPSCs were reduced by $42.35 \pm 4.0 \%\left(\mathrm{n}=7, \mathrm{t}_{(6)}=10.58\right.$, $\mathrm{P}<0.0001$, Fig. 4G) while, similarly to what observed for GluN2B-EPSCs evoked by optical cortico-striatal stimulation, $\alpha$-syn had no effect on the GluN2B currents ( $n=7, P>0.05$, Fig. 4G). This effect was observed at 5 after the $\alpha$-syn application. Notably, also in this case longer $\alpha$-syn applications did not further decrease the NMDA current and we found no effect of $\alpha$-syn on the GluN2B-EPSC at all time-points tested (at least $n=3$ for all time points, $P>0.05$, Fig. 4G).

\section{Monoclonal antibodies targeting $\alpha$-synuclein rescue the impairment of LTP}

Alpha-synuclein is a small protein with intrinsic tendency to aggregate. Oligomeric forms of the protein can impair neuronal function (Diogenes et al., 2012; Rockenstein et al., 2014). Accordingly, we found that $30 \mathrm{nM} \alpha$-syn cause LTP loss in SPNs by affecting the GluN2A NMDAR-mediated current. To counteract the effect of $\alpha$-syn on SPN LTP we used the monoclonal Ab Syn211, that recognizes all forms of human $\alpha$-syn (Giasson et al., 2000) and the $\mathrm{Ab}$ SynO2, which mainly 
recognizes early soluble oligomers and late aggregates amyloid fibrils of $\alpha$-syn (Majbour et al., 2016).

Incubation of the slices for 1 hour with the Syn211 Ab 1:10 alone did not affect LTP (158.3 $\pm 7.4 \%$, $\mathrm{n}=7, \mathrm{t}_{(6)}=6.863, \mathrm{P}<0.001$, Fig. $\left.5 \mathrm{~A}\right)$. However, incubation of the slices for 1 hour with the Syn $211 \mathrm{Ab}$ 1:10 in combination with $30 \mathrm{nM} \alpha$-syn was able to prevent the loss of LTP induced by $\alpha$-syn (153.0 $\pm 54.9 \%, \mathrm{n}=7, \mathrm{t}_{(6)}=10.52, \mathrm{P}<0.0001$, Fig. 5A). Conversely, a lower Ab concentration (1:100) was not sufficient to counteract the $\alpha$-syn effect on LTP amplitude; in fact, SPNs recorded in the presence of Syn211 Ab 1:100 plus $30 \mathrm{nM} \alpha$-syn showed no LTP (n=7, P>0.05, Supplementary Fig. 2).

We subsequently tested the effect of SynO2 Ab, which recognizes oligomeric forms of $\alpha$-syn more specifically than fibrillar forms. We found that while a very low concentration $(1: 10.000)$ of this $\mathrm{Ab}$ did not affect LTP per se $\left(143.2 \pm 3.8 \%, \mathrm{n}=7, \mathrm{t}_{(6)}=13.33, \mathrm{P}<0.0001\right.$, Fig. $\left.5 \mathrm{~B}\right)$, it rescued this form of synaptic plasticity when incubated in the presence of $30 \mathrm{nM} \alpha$-syn $\left(140.9 \pm 3.8 \%, \mathrm{n}=7, \mathrm{t}_{(6)}=12.01\right.$, $\mathrm{P}<0.0001$, Fig. 5B). Taken together these data suggest that Abs against $\alpha$-syn represent a valid tool for preventing the detrimental effect of $\alpha$-syn on striatal synaptic plasticity.

Next, we tested the ability of SynO2 and Syn 211 antibodies to capture $\alpha$-syn oligomers formed during incubation in Krebs solution. We conducted immunoprecipitation experiment with the samples collected at time 0 and 30 minutes with antibodies (SynO2 or Syn211) and estimated the $\alpha$ syn oligomers left in the sample using our oligomeric ELISA. Interestingly, when the samples were immunoprecipitated using SynO2, the supernatant contained very low levels of $\alpha$-syn oligomers with both high and low concentrations (Fig. 5C, left and right panel respectively), suggesting sequestration of $\alpha$-syn oligomers species with high efficacy (Fig. 5C, $* * \mathrm{P}<0.01$ ). Whereas, incubation with Syn211 antibody exerted a milder effect, and higher levels of $\alpha$-syn oligomers were detected in the supernatant (Fig. $5 \mathrm{C}, * * \mathrm{P}<0.01$ ). This dose-response nicely parallels that observed in their relative efficacy in rescuing the LTP impairment induced by $\alpha$-syn oligomers (Fig. 5A-B). 


\section{Incubation with alpha-synuclein specifically affects GluN2A localization at the post-synaptic membrane}

Notably, incubation of the slices with Syn211 Ab 1:10 or with SynO2 Ab 1:10.000 in combination with $30 \mathrm{nM} \alpha$-syn was sufficient to prevent the reduction of GluN2A levels in the postsynaptic compartment ( $\mathrm{n}=6-11, \mathrm{P}<0.01$, control vs $\alpha$-syn; $\mathrm{P}<0.05$, Syn211 plus $\alpha$-syn $v s \alpha$-syn; $\mathrm{P}<0.05$, SynO2 plus $\alpha$-syn $v s \alpha$-syn, Fig. 5D-E). In agreement with LTP results (see Supplementary Fig. 2), incubation of the slices with Syn211 Ab 1:100 did not revert the effect of $30 \mathrm{nM} \alpha$-syn on GluN2A synaptic levels ( $\mathrm{n}=7-11, \mathrm{P}<0.01$, control $v$ s $\alpha$-syn, Supplementary Fig. 3). No effect of $30 \mathrm{nM} \alpha$-syn on synaptic localization of GluN2B subunit of NMDAR or GluA1 and GluA2 subunits of AMPAR was observed either in absence or presence of co-incubation with Syn211 Ab 1:10 or with SynO2 Ab 1:10.000 (Supplementary Fig. 4A-D). Overall these results indicate that the observed decrease in GluN2A-NMDARs current induced by $\alpha$-syn is directly correlated to a reduction of the synaptic availability of this receptor subunit.

\section{Intrastriatal injections of alpha-synuclein protofibrils impairs visuo-spatial learning}

To test in vivo the effects of human $\alpha$-syn aggregates, we injected directly into the dorsal striatum preformed $\alpha$-syn fibrils (PFF), which is an already characterized progressive model of synucleinopathy. In fact, using a similar approach, a time-dependent spreading of $\alpha$-syn and neuronal degeneration has been reported about 9 months after the injection (Luk et al., 2012). Figure 6A shows the Transmission Electron Microscopy (TEM) analysis of protofibrils and shorter aggregates (putative oligomers) injected into the rat striatum. We decided to test the effects at 6 weeks post-injection (Fig. 7A-B); at this time point aggregates, and other $\alpha$-syn forms, are evidenced through their reaction with the phospho-Ser129hu- $\alpha$-Syn antibody (in red) and remained localized into the dorsal striatum (Fig. 6B-C). 
To test whether behavioural performance was affected, PBS- and $\alpha$-syn-injected rats were subjected to a visuo-spatial learning task. The task is based on a habituation/dishabituation paradigm in which animals during the habituation phase (from session 2 to session 7), through direct exploration, acquire information about 3 different objects and their spatial location in the arena (Fig. 7A-B). After this acquisition phase, one of the objects is displaced (session 8); the spatial preference for the displaced object (DO) over the non-displaced objects (NDO) is an index of spatial novelty detection. As reported in figure 7B, $\alpha$-syn-injected rats, as well as PBS-injected animals, explore the objects during the habituation phase, reducing the exploration time across sessions [Treatment $\left(\mathrm{F}_{1,15}=0.026 ; \mathrm{P}=0.874\right)$; Exploration $\left(\mathrm{F}_{5,75}=7.673 ; \mathrm{P}<0.0001\right)$; Exploration $\mathrm{x}$ Treatment $\left(\mathrm{F}_{5,75}=0.75\right.$; $\mathrm{P}=0.589$ )] (Fig. 7B). After the spatial displacement the PBS group showed a preference for DO as compared to NDO1 and NDO2, as expected; in contrast, the $\alpha$-syn-injected group did not show any preference for the DO [Treatment $\left(\mathrm{F}_{1,15}=0.152 ; \mathrm{P}=0.702\right) ; \mathrm{DO}\left(\mathrm{F}_{2,30}=3.387 ; \mathrm{P}=0.047\right) ; \mathrm{DO} \mathrm{x}$ Treatment $\left.\left(\mathrm{F}_{2,30}=3.347 ; \mathrm{P}=0.049\right)\right]$. This effect was not due to a general effect on object exploration, attention or motivation to explore novelty, as both groups showed a clear preference when the same object was substituted with a new one ( $\mathrm{SO})$ during the last testing session [Treatment $\left(\mathrm{F}_{1,15}=0.348\right.$; $\mathrm{P}=0.564) ; \mathrm{SO}\left(\mathrm{F}_{2,30}=15.153 ; \mathrm{P}<0.0001\right) ; \mathrm{SO} x$ Treatment $\left.\left(\mathrm{F}_{2,30}=0.87 ; \mathrm{P}=0.429\right)\right]$.

\section{Intrastriatal injections of alpha-synuclein aggregates impair the SPNs GluN2A-bearing}

\section{NMDAR-current}

We subsequently attempted to test the possible causal link among the presence of striatal $\alpha$-syn aggregates, the synaptic transmission deficits, as observed in the in vitro electrophysiological experiments using $\alpha$-syn oligomers, and the behavioural deficits of $\alpha$-syn-injected rats in performing the visuo-spatial learning task.

A group of human $\alpha$-syn- and sham-injected rats were sacrificed one week after the behavioural task. SPNs of the two groups were patch-clamped and EPSCs were evoked at $-80 \mathrm{mV}$ holding 
potential, in the presence of $50 \mu \mathrm{M}$ picrotoxin, and at $+40 \mathrm{mV}$, in picrotoxin plus $10 \mu \mathrm{M} \mathrm{CNQX}$, to calculate the NMDA/AMPA ratio of the synaptic current. Statistical analysis revealed that SPNs of $\alpha$-syn-injected rats presented reduced NMDA/AMPA ratio in respect to sham-injected animals $(\alpha-$ syn vs sham, $\mathrm{n}=5$ for each group, $\mathrm{t}$-test, $\mathrm{t}_{(8)}=6.6, \mathrm{P}<0.001$; Fig. $7 \mathrm{C}$ ). In addition, since $\alpha$-syn oligomers specifically reduced the GluN2A-bearing NMDAR-currents (Fig. 2C), EPSCs of SPNs from $\alpha$-syn- and sham-injected rats were also recorded at $+40 \mathrm{mV}$ holding current in the presence of CNQX plus $3 \mu \mathrm{M}$ ifenprodil to measure the GluN2A-EPSC amplitude. We found that in SPNs of $\alpha-$ syn-injected rats the GluN2A-current was significantly smaller than that of sham rats ( $\alpha$-syn $v s$ sham in ifenprodil, $\mathrm{t}$-test, $\mathrm{t}_{(11)}=2.2, \mathrm{P}<0.05$; Fig. 7D) confirming that the spreading of $\alpha$-syn aggregates into the striatum affects the striatal synaptic transmission by reducing the SPN GluN2ANMDA current with significant implications on behavioural learning.

\section{Behavioural effects produced by intrastriatal injection of oligomeric alpha-synuclein}

Intrastriatal injections of human $\alpha$-syn aggregates, containing protofibrils but also shorter forms (see Fig. 6A), clearly produced behavioural and synaptic alterations (See Fig. 7). These alterations could be due either to the action of oligomeric and monomeric $\alpha$-syn forms, similar to those applied in our in vitro experiments, or to the critical presence of fibrillary PFF forms of this protein. Thus, to better dissect the pathogenetic role of different species of $\alpha$-syn (protofibrils versus oligomers and monomers), we performed a similar in vivo intrastriatal injection with $\alpha$-syn cocktail mainly containing oligomers (OLIGO).

We injected the $\alpha$-syn cocktail ( $\alpha$-syn OLIGO) directly into the dorsal striatum (Fig. 8A-B), using the same protocol used for PFF. As previously indicated for PFF (see Fig. 6A), also the presence of $\alpha$-syn OLIGO has been detected by TEM (Fig. 8A). Electron Microscopy was always used to analyse the quality of $\alpha$-syn preparations before injection into the rat striatum confirming the 
presence of oligomers with very few protofibrils (Fig. 8A). In addition, $\alpha$-syn OLIGO has been confirmed by immunofluorescence with antibodies against $\alpha$-syn and phospho- $\alpha$-syn (Fig. 8B-C). As reported in Fig. 8D-F, $\alpha$-syn OLIGO-injected rats, as well as PBS-injected animals, explore the objects during the habituation phase, reducing the exploration time across sessions [Treatment $\left(\mathrm{F}_{1,10}=0.86 ; \mathrm{P}=0.375\right)$; Exploration $\left(\mathrm{F}_{5,50}=9.65 ; \mathrm{P}<0.0001\right)$; Exploration $\mathrm{x}$ Treatment $\left(\mathrm{F}_{5,50}=0.45\right.$; $\mathrm{P}=0.80)$ ] (Fig. 8D). The $\alpha$-syn OLIGO-injected group did not show any preference for the DO [Treatment $\left(\mathrm{F}_{1,10}=0.138 ; \mathrm{P}=0.702\right) ; \mathrm{DO}\left(\mathrm{F}_{2,20}=8.039 ; \mathrm{P}=0.0027\right) ; \mathrm{DO} \times$ Treatment $\left(\mathrm{F}_{2,20}=4.7\right.$; $\mathrm{P}=0.02)]$ and $\mathrm{SO}$ [Treatment $\left(\mathrm{F}_{1,10}=0.713 ; \mathrm{P}=0.41\right) ; \mathrm{SO}\left(\mathrm{F}_{2,20}=8.70 ; \mathrm{P}=0.0019\right) ; \mathrm{SO} \times$ Treatment $\left.\left(\mathrm{F}_{2,20}=3.99 ; \mathrm{P}=0.03\right)\right]$ (Fig. 8E-F), thus suggesting that it impaired both spatial and object novelty recognition, in the absence of major degeneration of $\mathrm{TH}$ positive terminals in the striatum (Supplementary Fig. 5).

Analysis of basal motor activity in the open field reveals that at this early stage neither animals injected with protofibrils nor animals injected with $\alpha$-syn OLIGO show significant changes of motor behaviour (Supplementary Fig. 6).

\section{DISCUSSION}

In this study we have investigated the effects of exogenous human $\alpha$-syn on synaptic transmission and plasticity of SPNs, the main neuronal subtype of the striatum. We obtained four novel findings: 1) $\alpha$-syn impairs LTP by altering the activity of the GluN2A-containing NMDARs in SPNs belonging to the direct and indirect basal ganglia pathways; 2) $\alpha$-syn produces a reduction of NMDAR-mediated currents in SPNs optically activated by stimulation of either cortical or thalamic afferents; 3 ) the application of $\alpha$-syn-neutralizing antibodies prevents the widespread impairment of striatal LTP; 4) intrastriatal injection of $\alpha$-syn PFF produces deficits in visuo-spatial learning and GluN2A NMDA receptor function; 5) the intrastriatal injection of oligomers ( $\alpha$-syn OLIGO) mimics the behavioural effects produced by PFF, causing an even more severe visuo-spatial 
alteration. These data suggest that oligomeric/monomeric forms play a more relevant role than fibrillary forms on striatal-dependent behaviour.

\section{Striatal dysfunction as an early effect of alpha-synuclein toxicity}

The complex alterations of the basal ganglia pathways in Parkinson's disease involve degeneration of nigral DA neurons that leads to reduced striatal DA content and impaired synaptic plasticity of SPNs (Calabresi et al., 2007; Gerfen and Surmeier, 2011). These changes are accompanied by accumulation of $\alpha$-syn (Spillantini and Goedert, 2016), exerting a toxic role in Parkinson's disease in its oligomeric forms (Conway et al., 1998; Winner et al., 2011; Rockenstein et al., 2014) rather than in the fibrillar state or when clustered into LBs (Ghosh et al., 2015).

Although it has been previously reported that in the hippocampus micromolar concentrations of extracellular $\alpha$-syn oligomers are able to impair LTP and to increase basal synaptic transmission through a NMDAR-mediated action (Diogenes et al., 2012), little is known on the direct effects of $\alpha$-syn oligomers on striatal neurotransmission, a fundamental player in the regulation of voluntary motor function in physiological conditions and in Parkinson's disease. Our findings, demonstrating widespread involvement of much lower (nanomolar) concentrations of $\alpha$-syn oligomers in the alteration of synaptic transmission in striatal SPNs, are particularly relevant given the role exerted by altered forms of this protein in Parkinson's disease (Polymeropoulos et al., 1997; Spillantini et al., 1997; Vekrellis et al., 2011). These results extend our previous findings demonstrating that $\alpha$ syn oligomers is able to block LTP of striatal ChIs at lower concentrations $(3 \mathrm{nM})$ respect to that effective on SPNs in the present study $(30 \mathrm{nM})$, by selectively modulating the GluN2D-expressing NMDA receptors (Tozzi et al., 2016). Taken together these findings suggest that increasing concentrations of $\alpha$-syn oligomers progressively affect NMDAR-mediated synaptic plasticity on distinct neuronal populations indicating that the vulnerability to this protein is cell- and regionspecific. 


\section{Distinct vulnerability of different NMDA receptor subunits to alpha-synuclein}

We found that $\alpha$-syn impairs NMDAR-dependent LTP in striatal SPNs with no detrimental effect on LTD, according to the observation that this latter form of synaptic plasticity in the striatum does not require NMDAR activation (Calabresi et al., 2007).

We observed that $\alpha$-syn affected SPNs glutamatergic transmission by selectively affecting GluN2Aexpressing NMDAR currents. This is a remarkable finding since NMDAR bearing the GluN2A receptor subunit is necessary for LTP induction (Paoletti et al., 2013). Accordingly, here we have shown that activity-dependent LTP in SPNs is blocked by antagonists acting on the GluN2A, but not GluN2B, NMDAR subunit. In line with these findings, our data rule out significant effects of $\alpha$ syn on currents mediated by GluN2B subunit of NMDAR and by AMPAR. Moreover, as evidenced by WB assay, $\alpha$-syn is able to lower the synaptic localization of GluN2A NMDAR subunit in striatal slices without affecting levels of other subunits of ionotropic glutamate receptors.

\section{Alpha-synuclein alters NMDAR-mediated excitatory transmission at SPNs of both direct and indirect pathways}

According to the classical view, striato-nigral SPNs express D1-like DA receptors, representing the direct pathway of the basal ganglia, while striato-pallidal SPNs express the D2-like DA receptors, representing neurons of the indirect pathway (Gerfen et al., 1990; Gerfen and Surmeier, 2011). This differential molecular expression of DA receptors is important for two functional reasons. Firstly, activation of D1 DA receptor signalling is required for LTP (Reynolds et al., 2001; Calabresi et al., 2007), while D2 DA receptors are mainly implicated in LTD (Kreitzer and Malenka, 2007; Shen et al., 2008). The second aspect regards their possible different role in the motor function. In fact, activation of the direct pathway by DA is thought to promote movement initiation, whereas the activation of the indirect pathway would be involved in inhibition of motor activity (Mink, 2003; 
Nambu, 2008; Gerfen and Surmeier, 2011). In apparent contrast with this dichotomous view, more recent studies propose a new vision of the basal ganglia functionality, based on signalling integration at different levels (Cui et al., 2013; Calabresi et al., 2014). Anatomical evidences reported the existence of "bridging collaterals", axon collaterals connecting neurons of the direct and indirect pathways (Cazorla et al., 2015). On the basis of these different theories, using striatal slices from dtTomato mice, we investigated whether the alteration of LTP caused by $\alpha$-syn occurs either in a single or in both sub-populations of SPNs. We found that the detrimental effect of $\alpha$-syn on LTP and on NMDAR synaptic currents is evident both in D1- and D2-expressing SPNs.

\section{Cortico-striatal and thalamo-striatal glutamatergic inputs are equally affected by alpha-} synuclein

Further evidence of the widespread toxic effect of $\alpha$-syn on striatal synapses come from our investigation of the NMDAR-mediated current in cortico-striatal and in thalamo-striatal pathways. The striatum receives glutamatergic inputs both from cerebral cortex and thalamus (McGeorge and Faull, 1989; Smith et al., 2004; Doig et al., 2010) and these two systems act in concert to regulate motor function differentially contributing to specific tasks. Cortical afferents projecting to the dorsal striatum are mainly involved in planning, learning and execution of motor behaviour (Jog et al., 1999), whereas the thalamo-striatal inputs modulate attention-related stimuli, being engaged in behavioural switching, reinforcement functions and attention set-shifting (Smith et al., 2011). Moreover, these two systems might differentially contribute, in Parkinson's disease patients, to the generation of glutamatergic dysfunction in the striatum. Thus, we have investigated a possible differential effect of $\alpha$-syn on cortico-striatal versus thalamo-striatal synapses. To this aim we used a distinct optical stimulation of the two pathways using para-horizontal brain slices. Compared to coronal slices, this particular tissue preparation allows to specifically discriminating the two projections converging on SPNs (Smeal et al., 2007). Interestingly, $\alpha$-syn caused a similar reduction 
of GluN2A NMDAR current evoked by selectively activating either cortical or thalamic fibres. This finding, together with previous experiments performed with electrical stimulation, supports the idea that $\alpha$-syn causes a widespread alteration of excitatory synaptic transmission within the striatum.

The widespread striatal synaptic dysfunction can be prevented by antibodies against alphasynuclein

Several studies described the consequences of the application of Abs against $\alpha$-syn, reporting improvement in intracellular $\alpha$-syn aggregates clearing, reduction of $\alpha$-syn fibrils propagation, together with attenuation of memory and neurodegenerative deficits (Spencer et al., 2017). In the attempt to clarify whether specific monoclonal Abs against $\alpha$-syn exert protective effects over synaptic plasticity, we employed Syn211 Ab and SynO2 Ab; both Abs recognizing all forms of human $\alpha$-syn, however, SynO2 Ab is much more potent than Syn211 Ab in binding $\alpha$-syn oligomers and fibrils (Giasson et al., 2000; Majbour et al., 2016). The depletion experiment we performed, confirmed that either Syn211 Ab or SynO2 Ab shows affinity for $\alpha$-syn oligomeric aggregates, with a different binding specificity between the two Abs. Interestingly, we found that, an overlapping dose-response effect of the two Abs in rescuing the LTP impairment induced by $\alpha$ syn in striatal SPNs. The two Abs also prevented the reduction of GluN2A expression levels caused by $\alpha$-syn. This protective effect is consistent with our observation that $\alpha$-syn alters LTP affecting NMDAR-mediated currents, in particular the GluN2A subunit. Interestingly, the PFF preparation injected intrastriatally in in vivo experiments contained both OLIGO and PFF aggregates.

\section{Differential effect of fibrillar versus oligomeric forms of alpha-synuclein on visuo-spatial learning}

Accumulating in vivo evidence showed that $\alpha$-syn protofibrils directly injected in the striatum lead to a time-dependent spreading accompanied by neuronal loss and motor deficits in mice within 9 
months after the injection (Luk et al., 2012). In this study we aimed at verifying whether the pathological interaction between $\alpha$-syn aggregates and NMDA receptors in the striatum led to impaired behavioural function; for this reason, we focused on an early time point when the aggregates remained localized within the striatum. To dissect the behavioural effects of $\alpha$-syn aggregates we used a visuo-spatial learning task that we have previously shown to rely on NMDA receptors activation in the dorsal striatum (De Leonibus et al., 2003; De Leonibus et al., 2005). $\alpha-$ syn PFF injected rats show impaired spatial novelty detection, although they normally explore the objects during the habituation phase and can discriminate a new object among familiar ones.

Interestingly, the intrastriatal injection of oligomers mimics the behavioural effects produced by PFF. This effect is even more severe than that one induced by PFF injection since while fibrils only affect the ability to discriminate the object displacement, oligomers impair both spatial and object novelty recognition. This is surprising considering that the PFF preparation injected intrastriatally in in vivo experiments contained both OLIGO and PFF aggregates and that the $\alpha$-syn cocktail producing the rapid synaptic and plastic alterations mainly contain oligomeric/monomeric forms, but little or no fibrils, as confirmed by the ThT analyses.

While the effect of in vivo injection of $\alpha$-syn on SPN NMDAR currents does not provide a direct mechanistic explanation that NMDAR changes are responsible of the described behavioural alteration, it confirms that $\alpha$-syn is able to produce molecular changes affecting the function of this receptor with possible behavioural consequences.

Our experiments in $\alpha$-syn-injected rats showed specific deficits in visuo-spatial exploration while locomotor activity was not affected respect to control animals. It possible that we failed to detect motor deficits because of the site and/or size of the injection within the striatum.

All together, these findings suggest that oligomeric $\alpha$-syn itself is harmful for synaptic plasticity and behavioural functions of the dorsal striatum. We speculate that further $\alpha$-syn aggregation into protofibrils might limit their direct impact within the area of injection, while favouring their 
spreading across brain regions as previously reported (Luk et al., 2012). Further studies are necessary to address this issue. The translational relevance of this preclinical data for PD are evidenced by findings in humans showing visuo-spatial learning deficit in de novo PD patients showing spatial location deficits (Pillon et al., 1997).

\section{Conclusions}

Although the role of $\alpha$-syn in the pathogenesis of Parkinson's disease has been deeply investigated, little is known on the effect of $\alpha$-syn on striatal synaptic transmission and related early behavioural alterations. Our research demonstrates a widespread and NMDAR-related dysfunction of striatal synaptic network induced by $\alpha$-syn. We report for the first time that $\alpha$-syn, through interaction with GluN2A-bearing NMDAR, impairs LTP in SPNs of the direct and indirect basal ganglia pathways and causes visuo-spatial learning deficit. The $\alpha$-syn-induced synaptopathy is also observed when neurons are optogenetically activated by either cortical or thalamic inputs. Notably, both plastic and behavioural alterations observed in our study do not seem to be dependent on dopaminergic degeneration. In fact, the plastic dysfunctions induced by acute in vitro application of $\alpha$-syn are not rescued by exogenous DA. Accordingly, the observed visuo-spatial learning deficits are not associated with a loss of dopaminergic terminals. Moreover, the open field analysis does not show overt motor impairment indicating that our in vivo model recapitulates an early stage of the disease. Finally, we provide evidence that antibodies neutralizing $\alpha$-syn are effective in blocking its detrimental actions. Taken together these data support the use of antibodies against $\alpha$-syn as a valid approach to preserve the functionality of synaptic plasticity in the striatal network.

FINANCIAL DISCLOSURES. All authors reported no biomedical financial interests or potential conflicts of interest. 
ACKNOWLEDGMENTS. This work was supported by grants from Fondazione Cariplo Grant 2014-0660 (FG and PC), MIUR-PRIN 2015FNWP34 (PC, FG and EDL), Ricerca Finalizzata RF2013-02356215 (PC and FG) and RF-2013-02357386 (BP). Valentina Durante at present has a fellowship from "Dipartimento Neuroscienze, psicologia, area del farmaco e salute del bambino, Università di Firenze", her position has been supported by "AIRAlzh Onlus-COOP Italia". This work/project was supported by a grant (V.G., P.C.) from the Fresco Parkinson Institute to New York University School of Medicine and The Marlene and Paolo Fresco Institute for Parkinson's and Movement Disorders, which was made possible with support from Marlene and Paolo Fresco. We thank Cristiano Spaccatini, Nadia Santo and Elisa Zianni for their excellent technical support.

SUPPLEMENTARY MATERIAL. Supplementary material is available at Brain online.

\section{REFERENCES}

Abeliovich A, Schmitz Y, Farinas I, Choi-Lundberg D, Ho WH, Castillo PE, et al. Mice lacking alpha-synuclein display functional deficits in the nigrostriatal dopamine system. Neuron 2000; 25(1): 239-52.

Arenkiel BR, Peca J, Davison IG, Feliciano C, Deisseroth K, Augustine GJ, et al. In vivo lightinduced activation of neural circuitry in transgenic mice expressing channelrhodopsin-2. Neuron 2007; 54(2): 205-18.

Cabin DE, Shimazu K, Murphy D, Cole NB, Gottschalk W, McIlwain KL, et al. Synaptic vesicle depletion correlates with attenuated synaptic responses to prolonged repetitive stimulation in mice lacking alpha-synuclein. J Neurosci 2002; 22(20): 8797-807.

Calabresi P, Maj R, Pisani A, Mercuri NB, Bernardi G. Long-term synaptic depression in the striatum: physiological and pharmacological characterization. J Neurosci 1992a; 12(11): 422433.

Calabresi P, Picconi B, Tozzi A, Di Filippo M. Dopamine-mediated regulation of corticostriatal synaptic plasticity. Trends Neurosci 2007; 30(5): 211-9.

Calabresi P, Picconi B, Tozzi A, Ghiglieri V, Di Filippo M. Direct and indirect pathways of basal ganglia: a critical reappraisal. Nat Neurosci 2014; 17(8): 1022-30.

Calabresi P, Pisani A, Mercuri NB, Bernardi G. Long-term Potentiation in the Striatum is Unmasked by Removing the Voltage-dependent Magnesium Block of NMDA Receptor Channels. Eur J Neurosci 1992b; 4(10): 929-35. 
Cazorla M, Kang UJ, Kellendonk C. Balancing the basal ganglia circuitry: a possible new role for dopamine D2 receptors in health and disease. Mov Disord 2015; 30(7): 895-903.

Conway KA, Harper JD, Lansbury PT. Accelerated in vitro fibril formation by a mutant alphasynuclein linked to early-onset Parkinson disease. Nat Med 1998; 4(11): 1318-20.

Cui G, Jun SB, Jin X, Pham MD, Vogel SS, Lovinger DM, et al. Concurrent activation of striatal direct and indirect pathways during action initiation. Nature 2013; 494(7436): 238-42.

De Leonibus E, Lafenetre P, Oliverio A, Mele A. Pharmacological evidence of the role of dorsal striatum in spatial memory consolidation in mice. Behav Neurosci 2003; 117(4): 685-94.

De Leonibus E, Manago F, Giordani F, Petrosino F, Lopez S, Oliverio A, et al. Metabotropic glutamate receptors 5 blockade reverses spatial memory deficits in a mouse model of Parkinson's disease. Neuropsychopharmacology 2009; 34(3): 729-38.

De Leonibus E, Oliverio A, Mele A. A study on the role of the dorsal striatum and the nucleus accumbens in allocentric and egocentric spatial memory consolidation. Learn Mem 2005; 12(5): 491-503.

De Leonibus E, Pascucci T, Lopez S, Oliverio A, Amalric M, Mele A. Spatial deficits in a mouse model of Parkinson disease. Psychopharmacology (Berl) 2007; 194(4): 517-25.

Ding J, Peterson JD, Surmeier DJ. Corticostriatal and thalamostriatal synapses have distinctive properties. J Neurosci 2008; 28(25): 6483-92.

Diogenes MJ, Dias RB, Rombo DM, Vicente Miranda H, Maiolino F, Guerreiro P, et al. Extracellular alpha-synuclein oligomers modulate synaptic transmission and impair LTP via NMDA-receptor activation. J Neurosci 2012; 32(34): 11750-62.

Doig NM, Moss J, Bolam JP. Cortical and thalamic innervation of direct and indirect pathway medium-sized spiny neurons in mouse striatum. J Neurosci 2010; 30(44): 14610-8.

Dunah AW, Wang Y, Yasuda RP, Kameyama K, Huganir RL, Wolfe BB, et al. Alterations in subunit expression, composition, and phosphorylation of striatal N-methyl-D-aspartate glutamate receptors in a rat 6-hydroxydopamine model of Parkinson's disease. Mol Pharmacol 2000; 57(2): 342-52.

El-Agnaf OM, Salem SA, Paleologou KE, Cooper LJ, Fullwood NJ, Gibson MJ, et al. Alphasynuclein implicated in Parkinson's disease is present in extracellular biological fluids, including human plasma. FASEB J 2003; 17(13): 1945-7.

Gallegos S, Pacheco C, Peters C, Opazo CM, Aguayo LG. Features of alpha-synuclein that could explain the progression and irreversibility of Parkinson's disease. Front Neurosci 2015; 9: 59. 
Gardoni F, Picconi B, Ghiglieri V, Polli F, Bagetta V, Bernardi G, et al. A critical interaction between NR2B and MAGUK in L-DOPA induced dyskinesia. J Neurosci 2006; 26(11): 291422.

Gerfen CR, Engber TM, Mahan LC, Susel Z, Chase TN, Monsma FJ, Jr., et al. D1 and D2 dopamine receptor-regulated gene expression of striatonigral and striatopallidal neurons. Science 1990; 250(4986): 1429-32.

Gerfen CR, Surmeier DJ. Modulation of striatal projection systems by dopamine. Annu Rev Neurosci 2011; 34: 441-66.

Ghiglieri V, Calabrese V, Calabresi P. Alpha-Synuclein: From Early Synaptic Dysfunction to Neurodegeneration. Front Neurol 2018; 9: 295.

Ghosh D, Singh PK, Sahay S, Jha NN, Jacob RS, Sen S, et al. Structure based aggregation studies reveal the presence of helix-rich intermediate during alpha-Synuclein aggregation. Sci Rep 2015; 5: 9228.

Giasson BI, Jakes R, Goedert M, Duda JE, Leight S, Trojanowski JQ, et al. A panel of epitopespecific antibodies detects protein domains distributed throughout human alpha-synuclein in Lewy bodies of Parkinson's disease. J Neurosci Res 2000; 59(4): 528-33.

Jocoy EL, Andre VM, Cummings DM, Rao SP, Wu N, Ramsey AJ, et al. Dissecting the contribution of individual receptor subunits to the enhancement of N-methyl-d-aspartate currents by dopamine D1 receptor activation in striatum. Front Syst Neurosci 2011; 5: 28.

Jog MS, Kubota Y, Connolly CI, Hillegaart V, Graybiel AM. Building neural representations of habits. Science 1999; 286(5445): 1745-9.

Kirik D, Rosenblad C, Bjorklund A. Characterization of behavioral and neurodegenerative changes following partial lesions of the nigrostriatal dopamine system induced by intrastriatal 6hydroxydopamine in the rat. Exp Neurol 1998; 152(2): 259-77.

Kreitzer AC, Malenka RC. Endocannabinoid-mediated rescue of striatal LTD and motor deficits in Parkinson's disease models. Nature 2007; 445(7128): 643-7.

Lim DH, Mohajerani MH, Ledue J, Boyd J, Chen S, Murphy TH. In vivo Large-Scale Cortical Mapping Using Channelrhodopsin-2 Stimulation in Transgenic Mice Reveals Asymmetric and Reciprocal Relationships between Cortical Areas. Front Neural Circuits 2012; 6: 11.

Luk KC, Kehm V, Carroll J, Zhang B, O'Brien P, Trojanowski JQ, et al. Pathological alphasynuclein transmission initiates Parkinson-like neurodegeneration in nontransgenic mice. Science 2012; 338(6109): 949-53. 
Majbour NK, Vaikath NN, van Dijk KD, Ardah MT, Varghese S, Vesterager LB, et al. Oligomeric and phosphorylated alpha-synuclein as potential CSF biomarkers for Parkinson's disease. Mol Neurodegener 2016; 11: 7.

McGeorge AJ, Faull RL. The organization of the projection from the cerebral cortex to the striatum in the rat. Neuroscience 1989; 29(3): 503-37.

Mellone M, Stanic J, Hernandez LF, Iglesias E, Zianni E, Longhi A, et al. NMDA receptor GluN2A/GluN2B subunit ratio as synaptic trait of levodopa-induced dyskinesias: from experimental models to patients. Front Cell Neurosci 2015; 9: 245.

Mink JW. The Basal Ganglia and involuntary movements: impaired inhibition of competing motor patterns. Arch Neurol 2003; 60(10): 1365-8.

Nambu A. Seven problems on the basal ganglia. Curr Opin Neurobiol 2008; 18(6): 595-604.

Nilsson MR. Techniques to study amyloid fibril formation in vitro. Methods 2004; 34(1): 151-60.

Paoletti P, Bellone C, Zhou Q. NMDA receptor subunit diversity: impact on receptor properties, synaptic plasticity and disease. Nat Rev Neurosci 2013; 14(6): 383-400.

Paxinos GW, C. . The Rat Brain Stereotaxic Coordinates. 5th Edn San Diego. Elsevier Academic Press 2005.

Pillon B, Ertle S, Deweer B, Bonnet AM, Vidailhet M, Dubois B. Memory for spatial location in 'de novo' parkinsonian patients. Neuropsychologia 1997; 35(3): 221-8.

Polymeropoulos MH, Lavedan C, Leroy E, Ide SE, Dehejia A, Dutra A, et al. Mutation in the alpha-synuclein gene identified in families with Parkinson's disease. Science 1997; 276(5321): 2045-7.

Reynolds JN, Hyland BI, Wickens JR. A cellular mechanism of reward-related learning. Nature $2001 ; 413(6851): 67-70$.

Rockenstein E, Nuber S, Overk CR, Ubhi K, Mante M, Patrick C, et al. Accumulation of oligomerprone alpha-synuclein exacerbates synaptic and neuronal degeneration in vivo. Brain 2014; 137(Pt 5): 1496-513.

Rodo C, Sargolini F, Save E. Processing of spatial and non-spatial information in rats with lesions of the medial and lateral entorhinal cortex: Environmental complexity matters. Behav Brain Res 2017; 320: 200-9.

Royston P. A Toolkit for Testing for Non-Normality in Complete and Censored Samples. Journal of the Royal Statistical Society Series D (The Statistician) 1993; 42(1): 37-43. 
Sciamanna G, Tassone A, Mandolesi G, Puglisi F, Ponterio G, Martella G, et al. Cholinergic dysfunction alters synaptic integration between thalamostriatal and corticostriatal inputs in DYT1 dystonia. J Neurosci 2012; 32(35): 11991-2004.

Shen W, Flajolet M, Greengard P, Surmeier DJ. Dichotomous dopaminergic control of striatal synaptic plasticity. Science 2008; 321(5890): 848-51.

Smeal RM, Gaspar RC, Keefe KA, Wilcox KS. A rat brain slice preparation for characterizing both thalamostriatal and corticostriatal afferents. J Neurosci Methods 2007; 159(2): 224-35.

Smith Y, Raju DV, Pare JF, Sidibe M. The thalamostriatal system: a highly specific network of the basal ganglia circuitry. Trends Neurosci 2004; 27(9): 520-7.

Smith Y, Surmeier DJ, Redgrave P, Kimura M. Thalamic contributions to Basal Ganglia-related behavioral switching and reinforcement. J Neurosci 2011; 31(45): 16102-6.

Spencer B, Valera E, Rockenstein E, Overk C, Mante M, Adame A, et al. Anti-alpha-synuclein immunotherapy reduces alpha-synuclein propagation in the axon and degeneration in a combined viral vector and transgenic model of synucleinopathy. Acta Neuropathol Commun 2017; 5(1): 7 .

Spillantini MG, Goedert M. Synucleinopathies: past, present and future. Neuropathol Appl Neurobiol 2016; 42(1): 3-5.

Spillantini MG, Schmidt ML, Lee VM, Trojanowski JQ, Jakes R, Goedert M. Alpha-synuclein in Lewy bodies. Nature 1997; 388(6645): 839-40.

Tanaka M, Kim YM, Lee G, Junn E, Iwatsubo T, Mouradian MM. Aggresomes formed by alphasynuclein and synphilin-1 are cytoprotective. J Biol Chem 2004; 279(6): 4625-31.

Tozzi A, de Iure A, Bagetta V, Tantucci M, Durante V, Quiroga-Varela A, et al. Alpha-Synuclein Produces Early Behavioral Alterations via Striatal Cholinergic Synaptic Dysfunction by Interacting With GluN2D N-Methyl-D-Aspartate Receptor Subunit. Biol Psychiatry 2016; 79(5): 402-14.

Vaikath NN, Majbour NK, Paleologou KE, Ardah MT, van Dam E, van de Berg WD, et al. Generation and characterization of novel conformation-specific monoclonal antibodies for alpha-synuclein pathology. Neurobiol Dis 2015; 79: 81-99.

Vastagh C, Gardoni F, Bagetta V, Stanic J, Zianni E, Giampa C, et al. N-methyl-D-aspartate (NMDA) receptor composition modulates dendritic spine morphology in striatal medium spiny neurons. J Biol Chem 2012; 287(22): 18103-14.

Vekrellis K, Xilouri M, Emmanouilidou E, Rideout HJ, Stefanis L. Pathological roles of alphasynuclein in neurological disorders. Lancet Neurol 2011; 10(11): 1015-25. 
Winner B, Jappelli R, Maji SK, Desplats PA, Boyer L, Aigner S, et al. In vivo demonstration that alpha-synuclein oligomers are toxic. Proc Natl Acad Sci U S A 2011; 108(10): 4194-9.

Wong YC, Krainc D. alpha-synuclein toxicity in neurodegeneration: mechanism and therapeutic strategies. Nat Med 2017; 23(2): 1-13.

Wu YW, Kim JI, Tawfik VL, Lalchandani RR, Scherrer G, Ding JB. Input- and cell-type-specific endocannabinoid-dependent LTD in the striatum. Cell Rep 2015; 10(1): 75-87.

\section{Figure legends}

Figure 1. Effect of $\alpha$-synuclein on the intrinsic membrane properties and synaptic plasticity of striatal SPNs. (A) Representative voltage traces (top) and current-voltage graph (bottom) recorded from SPNs during the injection of hyperpolarizing and depolarizing steps of current in control conditions ( $n=5)$ and in the presence of $30 \mathrm{nM} \alpha$-syn $(n=4)$ show no differences in the firing pattern discharge and intrinsic membrane properties of these neurons. (B) Examples traces (top) and time course (bottom) of the excitatory postsynaptic currents (EPSCs) measured before and 30 minutes after the application of the HFS protocol show no differences in the LTD induction in SPNs recorded from control slices $(n=6)$ and from slices incubated with $\alpha$-syn $(n=6)$. (C) Paired EPSC traces (top) and time-course graph of the EPSC amplitude (bottom) recorded in SPNs from control slices $(n=7)$ and in SPNs of slices incubated with $30 \mathrm{nM} \alpha$-syn $(\mathrm{n}=7)$ before and after the delivery of the HFS protocol show the block of LTP in SPNs recorded from slices treated with $\alpha$-syn. (D) Histogram showing the EPSC amplitude 30 min following the HFS protocol of SPNs recorded from control striatal slices and from slices incubated with increasing concentrations of $\alpha$-syn $(3 \mathrm{nM}, \mathrm{n}=6$, $10 \mathrm{nM}, \mathrm{n}=7,300 \mathrm{nM}, \mathrm{n}=6$ ). (E) EPSC traces (top) and time-course graph of the EPSC amplitude (bottom) measured in SPNs of control slices $(\mathrm{n}=7)$ and of slices incubated with $30 \mathrm{nM} \alpha$-syn in the presence of $30 \mu \mathrm{M}$ dopamine (DA) $(n=6)$ bath applied before and after the HFS protocol. Data are represented as mean \pm SEM. (F) Histogram of the ELISA shows time- and dose-dependent $\alpha$-syn 
oligomers; higher level of $\alpha$-syn oligomers were detected in the $300 \mathrm{nM} \alpha$-syn samples collected at 30 min. (G) ThT assay shows that little fluorescence was detected with the $30 \mathrm{nM}$ concentration, independently on the timing of sampling. In contrast, high fluorescence signal was measurable with the $300 \mathrm{nM}$ dose. Data are reported as percentage of fluorescence registered at $\mathrm{T} 0 \pm \mathrm{SEM} . * \mathrm{P}<0.05$, $* * \mathrm{P}<0.01, * * * \mathrm{P}<0.001$

Figure 2. Effect of alpha-synuclein on AMPAR- and NMDAR-mediated currents. (A) Example traces (top) and histogram (bottom) showing the AMPAR-mediated synaptic current of SPNs recorded in pharmacological isolation, in the presence of APV and picrotoxin, before and after the application of $30 \mathrm{nM} \alpha$-syn (n=6). (B) Example traces of EPSCs (top) evoked at +40 and $-80 \mathrm{mV}$ holding potential and histogram (bottom) showing the AMPAR RI of SPNs recorded in control conditions and in the presence of $\alpha$-syn $(n=6)$. (C) Histogram showing the averaged EPSC amplitude carried by the GluN2A-expressing NMDAR measured in SPNs in the presence of picrotoxin, CNQX and ifenprodil (open orange bar) and after subsequent addition of $30 \mathrm{nM} \alpha$-syn $(n=6)$ (filled cyan bar). The superimposed traces show EPSCs acquired at $+40 \mathrm{mV}$ holding potential of a SPN in the presence of picrotoxin + CNQX (black trace), picrotoxin + CNQX + ifenprodil (orange trace) and picrotoxin $+\mathrm{CNQX}+$ ifenprodil $+\alpha$-syn (cyan trace). (D, E) Histograms showing the averaged EPSC amplitudes carried by the GluN2B-expressing NMDAR measured in SPNs in the presence of picrotoxin, CNQX and NVP-AAM077 ( $\mathrm{n}=6)$ (D, open violet bar) or picrotoxin, CNQX and TCN-201 (n=6) (E, open violet bar) and in the presence of $30 \mathrm{nM} \alpha$-syn on this current $(n=6)$ (D-E, filled cyan bars). Superimposed traces showing EPSCs acquired from a single SPN following the application of picrotoxin + CNQX (D, black trace), picrotoxin + CNQX + NVP-AAM077 (D, violet trace), picrotoxin + CNQX + NVP-AAM077 + $\alpha$-syn (D, cyan trace) or picrotoxin + CNQX (E, black trace), picrotoxin + CNQX + TCN-201 (E, violet trace), picrotoxin + CNQX + TCN-201 $+\alpha$-syn $(\mathrm{E}$, cyan trace). (F, G) Representative WB images and histograms 
showing the effects of incubation (1 hour) of corticostriatal slices with $30 \mathrm{nM} \alpha$-syn on the synaptic localization of GluA1 and GluA2 AMPAR subunits (n=8) (F) and GluN2A and GluN2B NMDAR subunits $(n=6)(G)$. Data are normalized against tubulin and are shown in the graph as mean \pm SEM. $* \mathrm{P}<0.05,{ }^{* *} \mathrm{P}<0.01$.

\section{Figure 3. Effect of alpha-synuclein on SPNs of the direct and indirect basal ganglia pathway.}

(A) Images of three regions acquired from a striatal slice of a transgenic Drd1a-tdTomato mouse showing fluorescent D1R-positive neurons (left, in red pseudocolor and top right) and a nonfluorescent (putative D2R-positive) SPN (bottom right). (B) Example traces and time-course graph showing the LTP induced in both fluorescent $(n=6)$ and non-fluorescent $(n=6)$ SPNs after the delivery of the HFS protocol. (C, D) EPSC pairs (top) and time-courses graphs of the EPSC amplitude (bottom) showing that $\alpha$-syn is able to block the LTP in both D1R-positive (n=5) (C) and putative D2R-positive SPNs (n=5) (D) following the HFS protocol. (E, F) Histograms showing the mean EPSC amplitudes of the NMDAR-mediated synaptic currents measured in pharmacological isolation in D1R-positive $(n=6)(E)$ and in putative D2R-positive SPNs $(n=4)(F)$ both in control condition and after the application of $30 \mathrm{nM} \alpha$-syn. The superimposed traces show EPSCs acquired from a D1R-positive SPN (E) and putative D2R-positive SPN (F) in the presence of CNQX plus picrotoxin and in CNQX plus picrotoxin plus $\alpha$-syn. Note that $\alpha$-syn is able to reduce the NMDARmediated current in a similar manner in these neuronal sub-populations. Data are represented as mean \pm SEM. $* \mathrm{P}<0.05, * * \mathrm{P}<0.01$

Figure 4. Effect of alpha-synuclein on SPNs activated by optical stimulation of cortico-striatal and thalamo-striatal glutamatergic afferents. (A) Representative para-horizontal brain slice including cortical, striatal and thalamic regions and (B) scheme of a para-horizontal slice showing the regions of the cortex and the thalamus where optical stimulation can activate glutamatergic 
afferents to SPNs recorded in the striatum. $(\mathbf{C}, \mathbf{F})$ Histograms of the SPNs mean NMDA-EPSC amplitudes optically stimulated in the cortex (C) or in the thalamus (F) in the presence of $3 \mu \mathrm{M}$ ifenprodil to isolate the GluN2A-EPSC ( $n=6$ cortex, $n=5$ thalamus) (orange open bar) or in the presence of $10 \mu \mathrm{M}$ TCN-201 $(\mathrm{n}=6)$ to isolate the GluN2B-EPSC (violet open bar). Data are presented as percentage of the control NMDAR-EPSCs recorded in the presence of picrotoxin and CNQX (dotted line). (D, G) Histograms of the averaged GluN2A- and GluN2B-EPSC amplitudes optically stimulated in the cortex (D) or in the thalamus (G) measured in SPNs in the presence of picrotoxin, CNQX, ifenprodil (orange outlined bars) without $(\mathrm{n}=6)$ and with $30 \mathrm{nM} \alpha$-syn (orange outlined, cyan filled bar) and picrotoxin, CNQX, TCN-201 (violet outlined bars) without ( $\mathrm{n}=6)$ and with $30 \mathrm{nM} \alpha$-syn (violet outlined, cyan filled bar). $\alpha$-syn (n=4-6) is applied for 2,5 and $10 \mathrm{~min}$. Data are presented as percentage of the control GluN2A- and GluN2B-EPSCs. (E, H) Top, superimposed EPSCs evoked by optical stimulation of the cortex $(\mathrm{E})$ or the thalamus $(\mathrm{H})$ acquired from a SPN in the presence of picrotoxin, CNQX, ifenprodil (orange trace) and after further the application of $30 \mathrm{nM} \alpha$-syn (cyan trace). Bottom, EPSCs acquired from a SPN in the presence of picrotoxin, CNQX, TCN-201 (violet trace) and after further the application of $30 \mathrm{nM} \alpha$-syn (cyan trace). Note that $\alpha$-syn significantly reduces the GluN2A- but not GluN2B-EPSC. Data are represented as mean $\pm \mathrm{SEM} . * * \mathrm{P}<0.01, * * * \mathrm{P}<0.001$

\section{Figure 5. Action of monoclonal antibodies against $\alpha$-synuclein on SPNs synaptic plasticity. (A)}

Time-course graph showing the EPSC amplitudes of SPNs recorded before and after the LTP induction by HFS in slices incubated for 1 hour with $30 \mathrm{nM} \alpha$-syn and in slices treated with Syn 211 $\mathrm{Ab}(1: 10)$ alone $(\mathrm{n}=7)$ or with Syn211 Ab (1:10) plus $\alpha$-syn $(\mathrm{n}=7)$. (B) Time-course of the SPNs EPSC amplitudes recorded before and after the HFS protocol in slices treated with $\alpha$-syn and in SPNs in the presence of SynO2 Ab alone $(1: 10.000)(n=7)$ or SynO2 Ab $(1: 10.000)$ plus $\alpha$-syn $(n=7)$. Note that the treatment with Syn211 or SynO2 Abs is able to restore the LTP in the presence 
of $\alpha$-syn. (C) Immunoprecipitation experiment with the samples collected at time 0 and 30 min with antibodies (SynO2 or Syn211) estimates the $\alpha$-syn oligomers left in the sample using oligomeric ELISA. (D, E) WB analysis for GluN2A subunit of the NMDAR in the postsynaptic fraction (TIF) obtained from striatal slices incubated with $\alpha$-syn alone (30 nM) or with $\alpha$-syn plus Syn211 (1:10) or Syn-O2 (1:10.000) antibodies and control slices. $\alpha$-syn reduced GluN2A localization at postsynaptic sites, while treatment with two different anti- $\alpha$-syn antibodies was able to rescue GluN2A levels in the TIF. Data are normalized against tubulin and are shown in the graph as mean \pm SEM. $* \mathrm{P}<0.05, * * \mathrm{P}<0.01$.

Figure 6. Intra-striatal PFF alpha-synuclein injection. (A) Representative TEM image of preformed fibrils (PFF) preparation injected in rat striatum. (B, C) Representative brain slice of animals injected with $\alpha$-syn in the striatum. Immunofluorescence for $\alpha$-syn and phospho-Ser129-hu$\alpha$-Syn. High levels of phosphor-Ser129-hu- $\alpha$-Syn are detectable at the injection site within the striatum, with no spreading in the surrounding brain regions. (B) A higher magnification (60x) evidences the $\alpha$-syn aggregates positive for phospho-Ser129hu- $\alpha$-Syn. (B) Representative brain slice of animals injected with PBS in the striatum. No positive staining of phospho-Ser129-hu- $\alpha-$ Syn is detectable in the striatum of the control group at low and high magnification (C).

Figure 7. Behavioural and electrophysiological alterations in rats injected with human alphasynuclein. (A) Schematic representation of the visuo-spatial novelty reaction task. Rats were habituated to the empty arena (S1). During the habituation phase they were allowed to explore for 6 consecutive sessions (S2-S7) three different objects kept in constant position. Spatial learning was tested by displacing the position of two objects, the blu one (Displaced object-DO), and comparing its relative preference as compared to the non-displaced objects (NDO), the black and the purple once (S8). Animals were given a further session to habituate to the spatial change (S9). During the 
last session object learning was tested by substituting one of the three objects (the blu one, substituted object-SO) with a new one (orange), and the other two objects with identical copies, to avoid the use of olfactory cues for object recognition (non-substituted objects-NSO) (S10). (B) Histograms showing the mean time (s) objects exploration during the habituation phase by control and $\alpha$-syn rats (left panel); Re-exploration index of displaced DO and NDO objects after the spatial change, showing that control, but not $\alpha$-syn rats, prefer DO vs. NDO (center panel); Re-exploration index of substituted SO and NSO objects after the object change, showing that both control and $\alpha$ syn rats preferred SO vs. NSO (right panel). ${ }^{*} \mathrm{P}<0.05 \mathrm{DO} / \mathrm{SO}$ vs. NDO/NSO, within group; \# $\mathrm{P}<0.05 \alpha$-syn vs. control, Duncan post-hoc test. DO: displaced objects; NDO: non-displaced objects; SO: substituted object; NSO: non-substituted object. Control rats, n=9, $\alpha$-syn rats, n=8. (C) Histogram showing the NMDA/AMPA ratio measured in SPNs of $\alpha$-syn- $(n=5)$ and of shaminjected (control) rats $(\mathrm{n}=5)$. Traces showing an EPSC measured at $+40 \mathrm{mV}$ and $-80 \mathrm{mV}$ holding potential in a SPN of a control and $\alpha$-syn rat. (D) Histogram showing the NMDA-current amplitude of SPNs from $\alpha$-syn- $(n=5)$ and control rats $(n=5)$ before (dashed line) and after the application of ifenprodil to isolate the GluN2A-mediated NMDA-current. Traces showing an EPSC measured at $+40 \mathrm{mV}$ holding potential in a SPN of a control and $\alpha$-syn rat before and after the application of ifenprodil. $* \mathrm{P}<0.05, * * \mathrm{P}<0.01$

Figure 8. Intra-striatal oligomeric alpha-synuclein injection and behavioural alterations. (A) Representative TEM image of OLIGO $\alpha$-syn preparation injected in rat striatum showing a majority of small oligomers with very few protofibrils. (B-C) Representative images of animals injected with $\alpha$-syn in the striatum. Immunofluorescence for hu- $\alpha$-syn (B) and phospho-Ser129-hu- $\alpha$-Syn (C). 40x magnification images evidences that high levels of hu- $\alpha$-syn (B) and phosphor-Ser129-hu- $\alpha-$ Syn (C) are detectable within the striatum. Representative images of animals injected with PBS in the striatum (B, C). No positive staining of phospho-Ser129-hu- $\alpha-S y n$ (C) is detectable in the 
striatum of the control group at 40x magnification. Arrows indicate hu- $\alpha$-syn and phospho-Ser129hu- $\alpha$-Syn positive cells; scale bars: $50 \mu \mathrm{m}$. (D-F) Histograms showing the mean time (s) objects exploration during the habituation phase by control and oligomeric $\alpha$-syn rats. Re-exploration index of displaced DO and NDO objects after the spatial change, showing that control, but not $\alpha$-syn rats, prefer DO vs. NDO (center panel). Re-exploration index of substituted SO and NSO objects after the object change, showing that both control and $\alpha$-syn rats preferred SO vs. NSO (right panel). Control rats, $\mathrm{n}=6$, oligomeric $\alpha$-syn rats, $\mathrm{n}=6$. ${ }^{*} \mathrm{P}<0.05 \mathrm{DO} / \mathrm{SO}$ vs. NDO/NSO, within group, Duncan post-hoc test. DO: displaced objects; NDO: non-displaced objects; SO: substituted object; NSO: non-substituted object. 
A
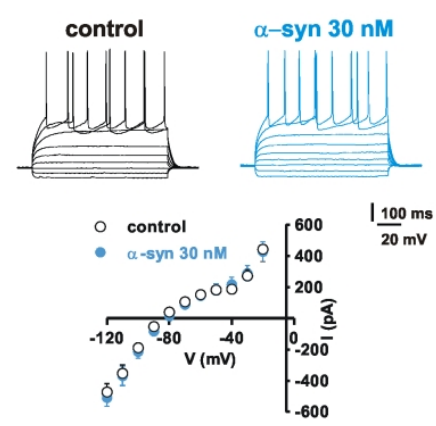

C
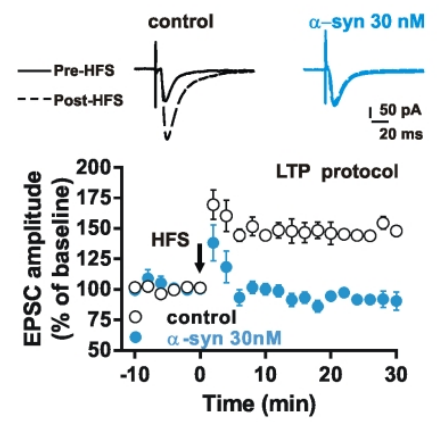

E
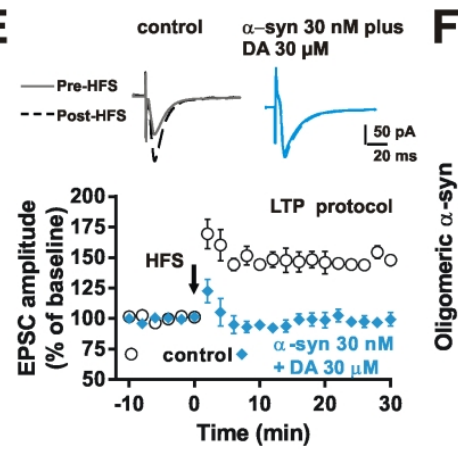

F
B
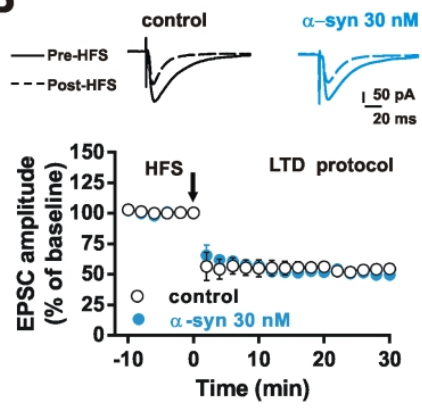

D

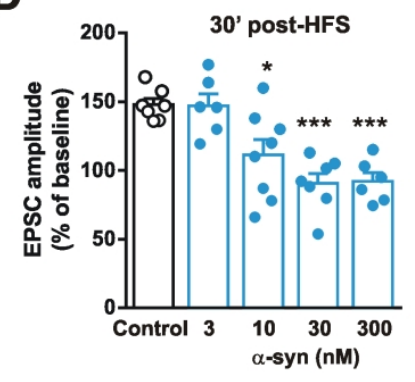

G

ELISA

ThT Assay
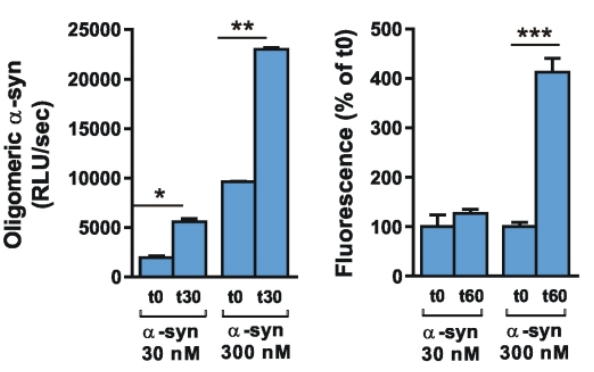

Fig. 1 

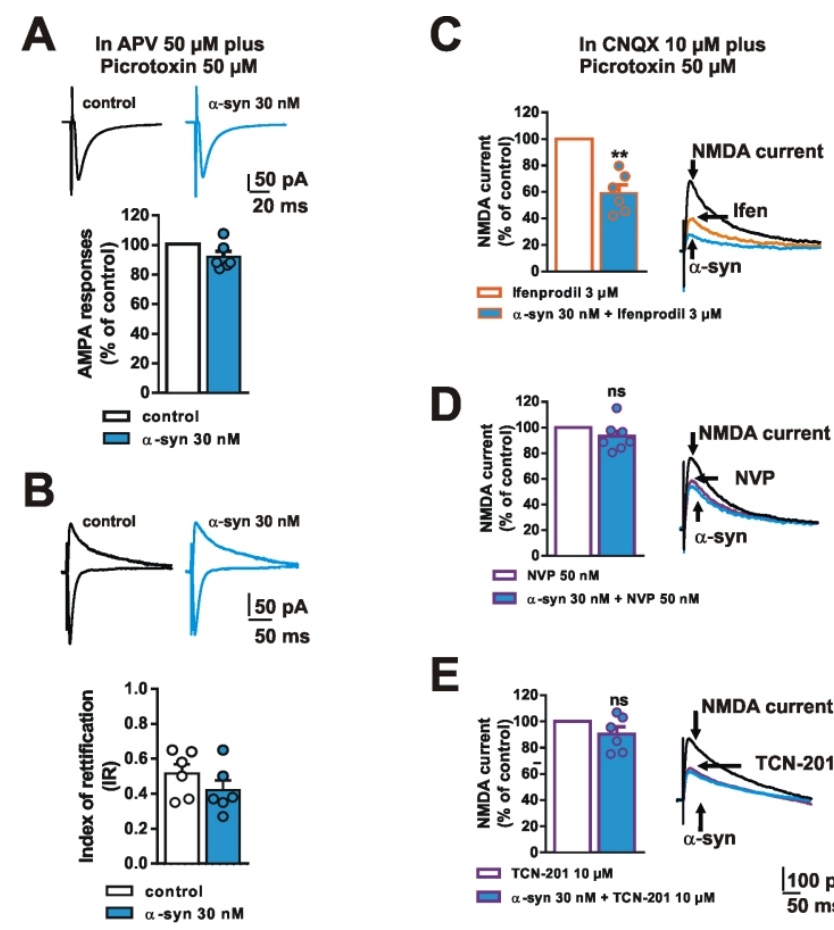

E
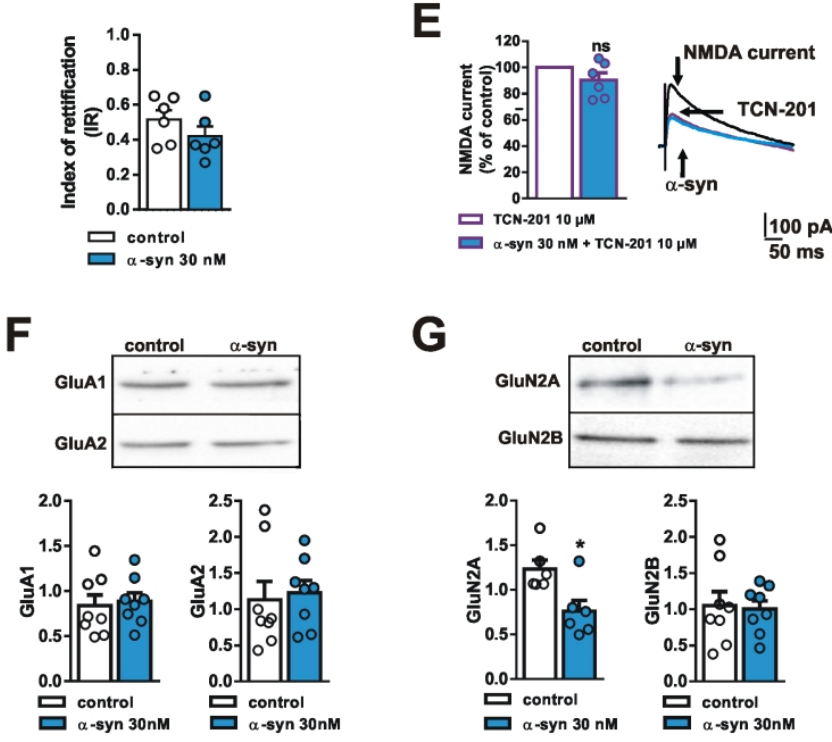

Fig. 2 
A
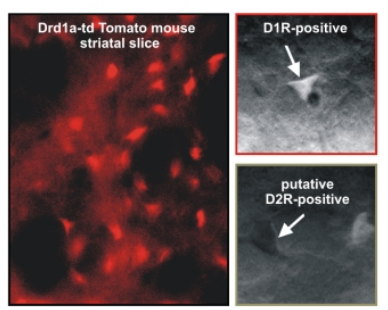

C

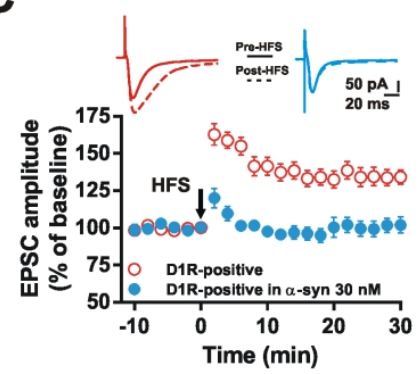

$\mathbf{E}$
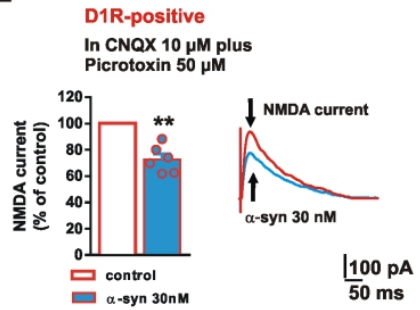

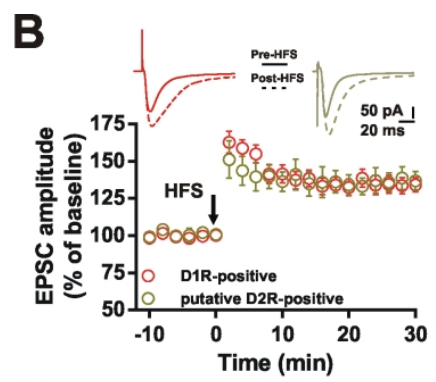

D

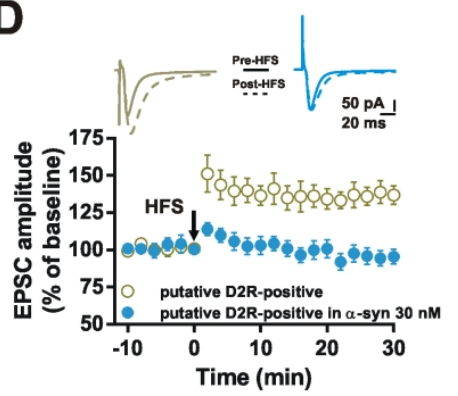

$\mathbf{F}$

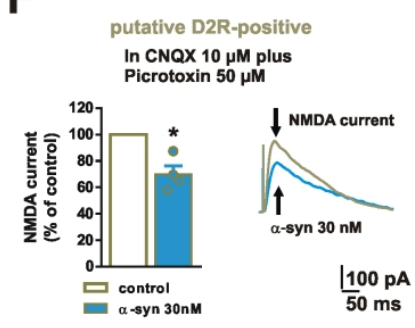

Fig. 3 
A
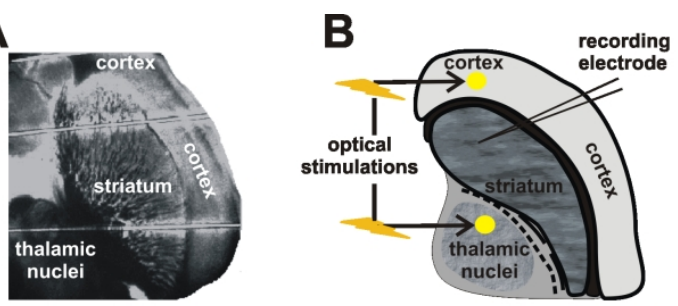

CORTEX

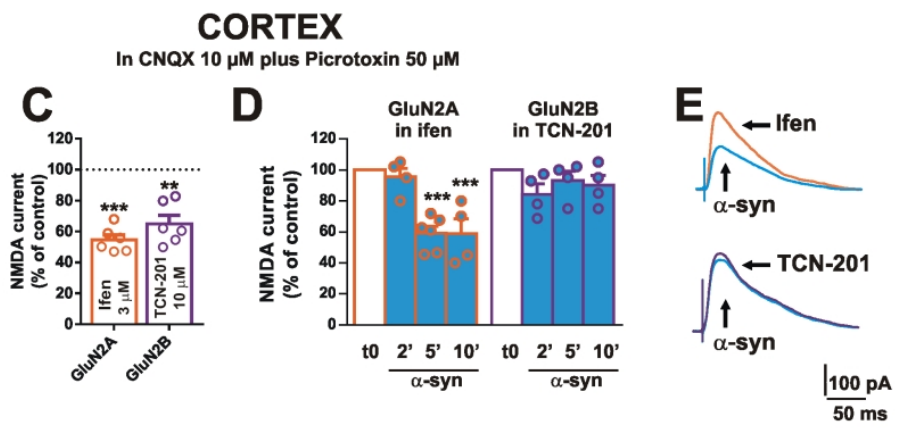

THALAMUS

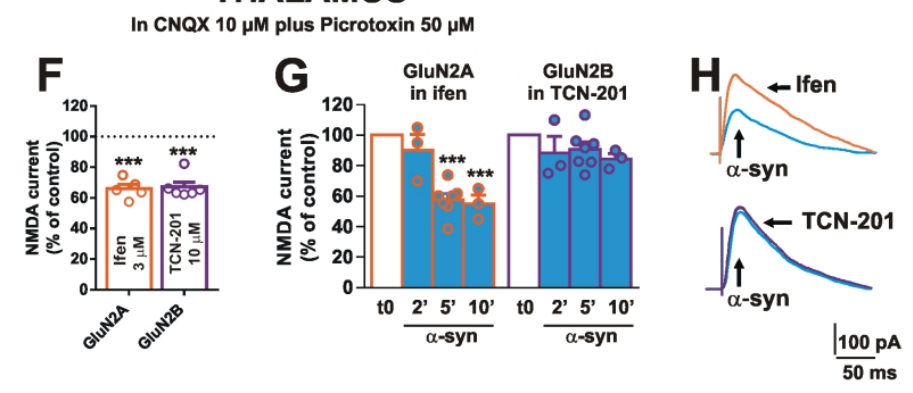

Fig. 4 
A

- $\alpha$-syn 30nM $\odot$ Syn211(1:10) - Syn211 (1:10) plus $\alpha$-syn30 nM

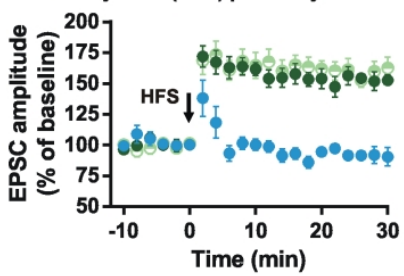

C

Depletion experiment
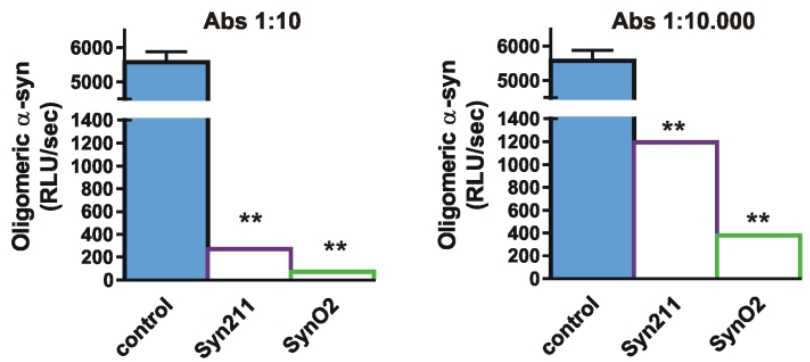

D

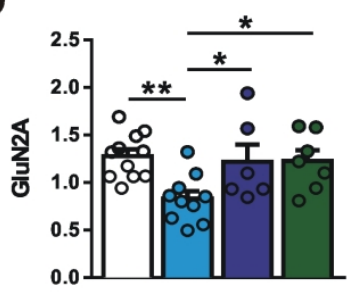

E
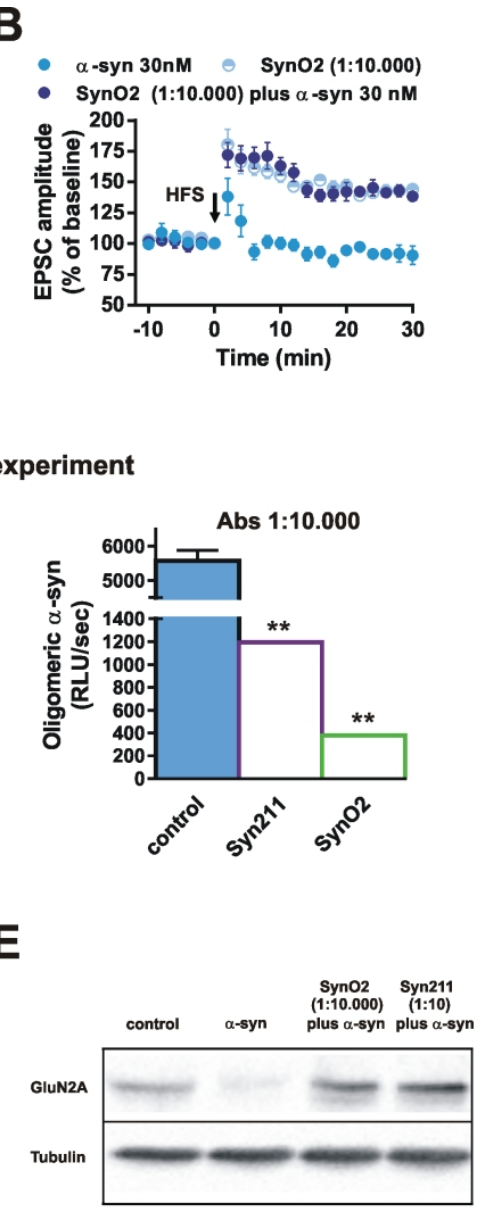

control

$\alpha-\operatorname{syn} 30 \mathrm{nM}$

Syn02 (1:10.000) plus $\alpha$-syn $30 \mathrm{nM}$

Syn211 (1:10) plus $\alpha$-syn $30 \mathrm{nM}$ 

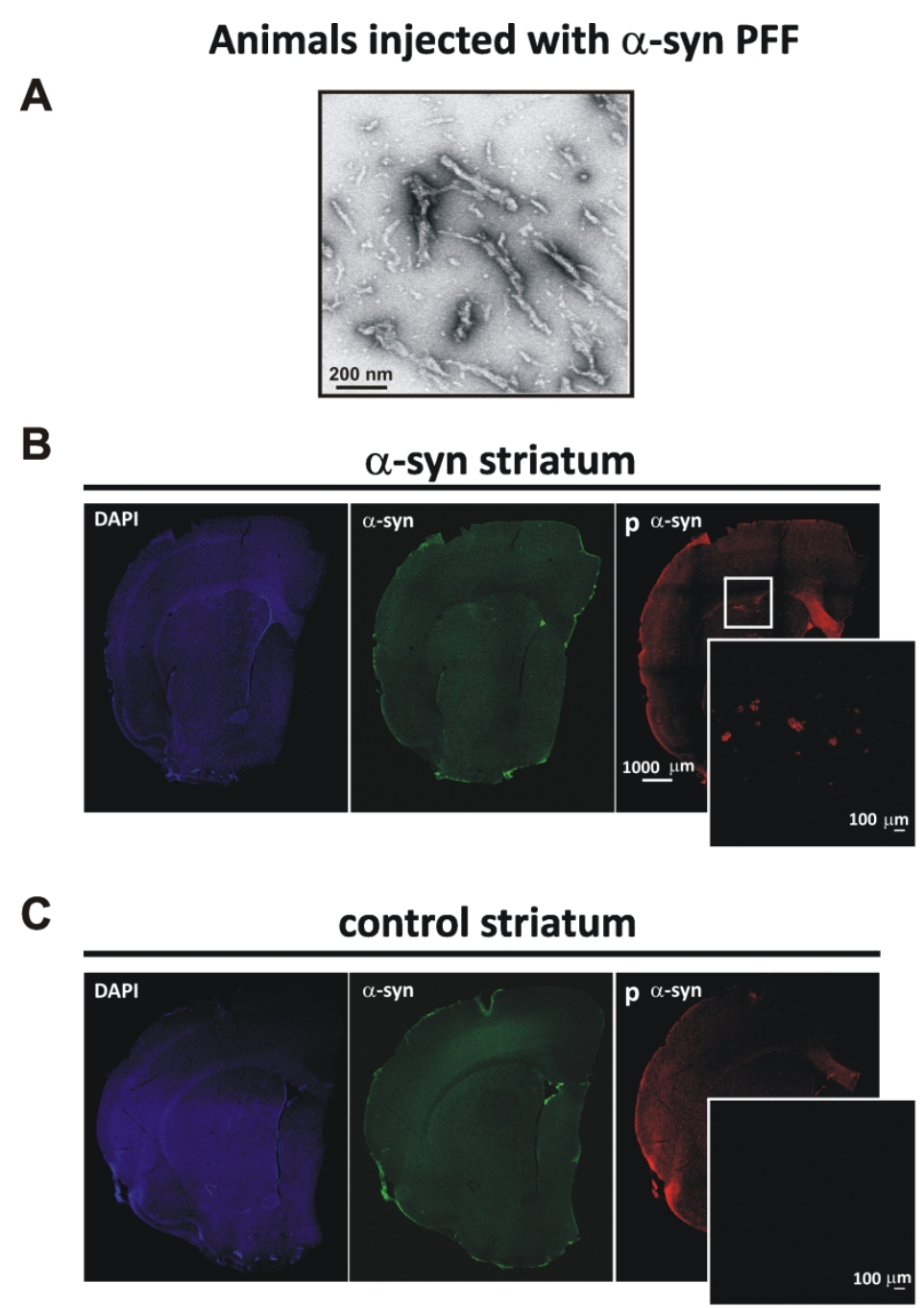

Fig. 6 

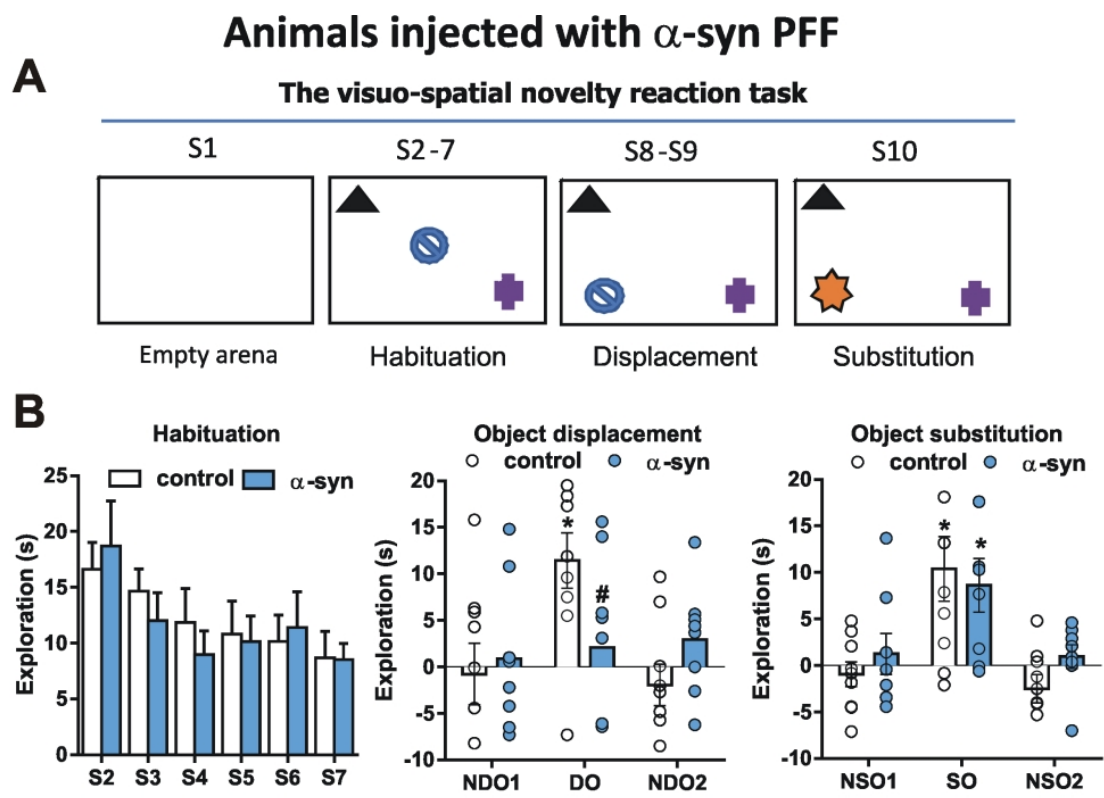

C

D In CNQX $10 \mu \mathrm{M}$ plus Picrotoxin $50 \mu \mathrm{M}$
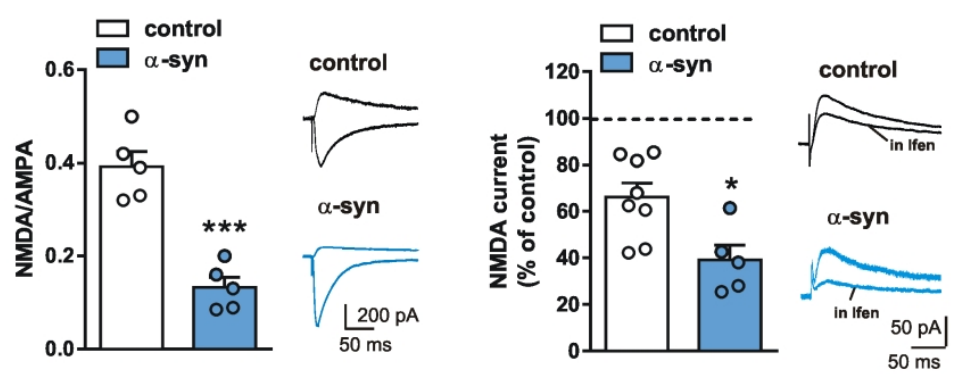

Fig. 7 


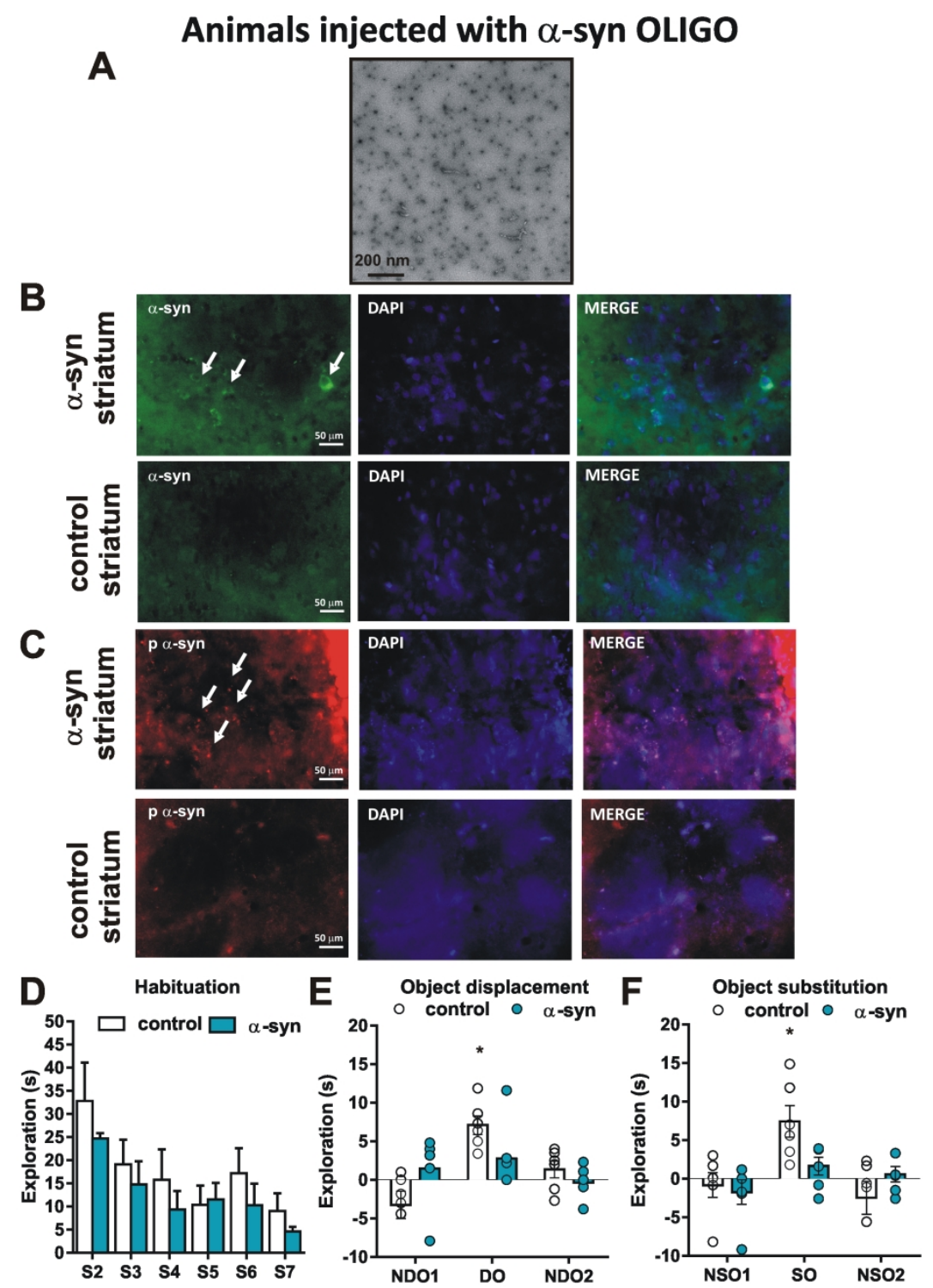

Fig. 8 


\section{Alpha-synuclein targets GluN2A NMDA receptor subunit causing striatal synaptic dysfunction and visuo-spatial memory alteration}

Durante $\mathrm{V}^{1}$, de Iure $\mathrm{A}^{1,2}$, Loffredo $\mathrm{V}^{3,4}$, Vaikath $\mathrm{N}^{5}$, De Risi $\mathrm{M}^{6}$ Paciotti $\mathrm{S}^{7}$, Quiroga-Varela $\mathrm{A}^{1}$, Chiasserini $\mathrm{D}^{1}$, Mellone $\mathrm{M}^{8}$, Mazzocchetti $\mathrm{P}^{1}$, Calabrese $\mathrm{V}^{1}$, Campanelli $\mathrm{F}^{1}$, Mechelli $\mathrm{A}^{1}$, Di Filippo $\mathrm{M}^{1}$, Ghiglieri $\mathrm{V}^{9,10}$, Picconi $\mathrm{B}^{2}$, El-Agnaf $\mathrm{OM}^{5}$, De Leonibus $\mathrm{E}^{3,6}$, Gardoni $\mathrm{F}^{8}$, Tozzi $\mathrm{A}^{7,9}$, and Calabresi $\mathrm{P}^{1,9^{*}}$

1. Neurological Clinic, Department of Medicine, Hospital Santa Maria della Misericordia, University of Perugia, Perugia, Italy;

2. Laboratory of Experimental Neurophysiology, IRCCS San Raffaele Pisana, Rome, Italy

3. Institute of Cellular Biology and Neurobiology, National Research Council, Monterotondo (Rome), Italy;

4. PhD Program in Behavioral Neuroscience, Sapienza University of Rome, Italy;

5. Neurological Disorders Research Center, Qatar Biomedical Research Institute (QBRI), Hamad Bin Khalifa University (HBKU), Qatar Foundation, Doha, Qatar;

6. Telethon Institute of Genetics and Medicine, Telethon Foundation, Pozzuoli (NA), Italy;

7. Department of Experimental Medicine, Section of Physiology and Biochemistry, University of Perugia, Perugia, Italy;

8. Department of Pharmacological and Biomolecular Sciences, University of Milan, Italy;

9. Laboratory of Neurophysiology, Santa Lucia Foundation, IRCCS, Rome, Italy;

10. Department of Philosophy, Human, Social and Educational Sciences, University of Perugia, Perugia, Italy.

Correspondence to:

Professor Paolo Calabresi

Neurological Clinic, Department of Medicine, University of Perugia,

Hospital Santa Maria della Misericordia,

06132 - S. Andrea delle Fratte, Perugia, Italy

Phone +39-075-578-4230; Fax +39-075-578-4229

Email: paolo.calabresi@unipg.it

Running title: Alpha-synuclein in synaptic transmission 


\section{Supplementary Figures}

\section{Supplementary Figure 1. The induction of SPNs LTP depends on NMDA receptor expressing} the GluN2A subunit. Example traces (top) and time-course of the EPSC amplitudes (bottom) of SPNs recorded in control slices, in slices treated with ifenprodil, or TCN-201 or NVP-AAM077 before and after the application of the HFS protocol. Note the presence of LTP in control slices and in slices treated with ifenprodil and the absence of LTP in SPNs recorded from slices treated with TCN-201 or NVP-AAM077. Data are represented as mean \pm SEM.

Supplementary Figure 2. Syn211 Ab (1:100) is not able to counteract the detrimental effect of 30 nM $\alpha$-synuclein. Time-course graph of the EPSC amplitudes recorded in SPNs of slices incubated with $30 \mathrm{nM} \alpha$-syn and in SPNs of slices treated with Syn $211 \mathrm{Ab}(1: 100)$ plus $\alpha$-syn. Note that Syn211 Ab (1:100) is not able to recover the detrimental effect of $30 \mathrm{nM} \alpha$-syn on LTP. Data are represented as mean $\pm \mathrm{SEM}$.

Supplementary Figure 3. SynO2 Ab recognizing oligomeric a-synuclein more specifically than Syn211 Ab at a higher concentration of $\alpha$-synuclein. $\alpha$-syn was dissolved in oxygenated Ringer solution at a concentration of $300 \mathrm{nM}$ and incubated up to 60 minutes. At different time points $(\theta$, $15,30,60$ minutes) aliquots of the solution were taken ( $200 \mathrm{ng}$ ) and separated using non-reducing SDS PAGE. Proteins were transferred on a blotting membrane and incubated with different antibodies. At this concentration $\alpha$-syn aggregated in SDS-resistant dimers, trimer, tetramers similarly to the concentration having effect on SPNs $(30 \mathrm{nM})$. Additionally, high molecular weight oligomers were also present. The preferential binding of the two antibodies was similar to that at 30 $\mathrm{nM}$, with the SynO2 antibody recognizing oligomeric $\alpha$-syn more specifically than Syn211. 
Supplementary Figure 3. Syn211 Ab (1:100) does not revert the effect of $\alpha$-syn on the postsynaptic localization of NMDAR GluN2A subunit. WB analyses from control slices, slices treated with $30 \mathrm{nM} \alpha$-syn or with $\alpha$-syn plus Syn211 Ab (1:100) showed no change in the postsynaptic levels of NMDAR GluN2A induced by the Ab. Data are normalized against tubulin and are shown in the graph as mean \pm SEM.

Supplementary Figure 4. No effect of $\alpha$-syn in presence or absence of Syn211 or SynO2 Abs on the postsynaptic localization of NMDAR GluN2B and AMPAR GluA1 and GluA2 subunits. (A-D) WB analyses from control slices, slices treated with $30 \mathrm{nM} \alpha$-syn or with $\alpha$-syn plus Syn 211 $\mathrm{Ab}(1: 10)$ or with $\alpha$-syn plus SynO2 Ab (1:10.000) showed no change in the postsynaptic levels of NMDAR GluN2B and AMPAR GluA1 and GluA2 subunits. Data are normalized against tubulin and are shown in the graph as mean \pm SEM.

Supplementary Figure 5. Representative images of animals injected with alpha-synuclein oligomers in the striatum; immunofluorescence for hu- $\alpha$-syn and TH. (A-B) 40x magnification images evidences that hu- $\alpha$-syn (A) is detectable within the striatum, while $\mathrm{TH}$ expression is comparable to that of control mice (B). Arrows indicate hu- $\alpha$-syn positive cells; scale bars: $50 \mu \mathrm{m}$.

Supplementary Figure 6. Motor behaviour alterations in rats injected with human oligomeric and PFF alpha-synuclein. Histograms showing the mean distance travelled (cm), immobility time (s) and maximal speed $(\mathrm{cm} / \mathrm{s})$ across time intervals in the empty open field during the first session (S1) of the visuo-spatial task for animals injected with protofibrils ( $\alpha$-syn PFF) (A) $\alpha$-syn, oligomeric ( $\alpha$-syn OLIGO) (B) and relative control (PBS) injected animals. No significant group effects were detected. 


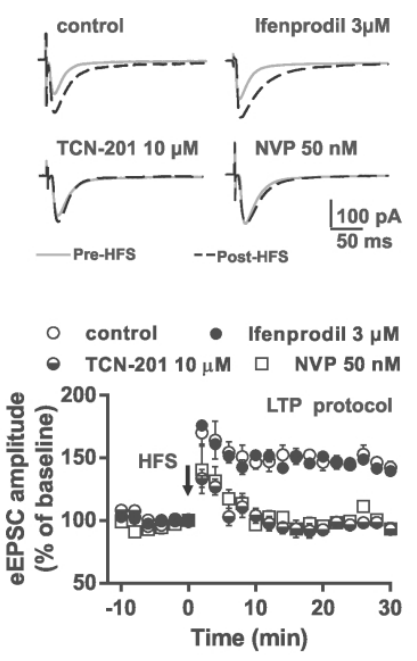

Supplementary Fig. 1 


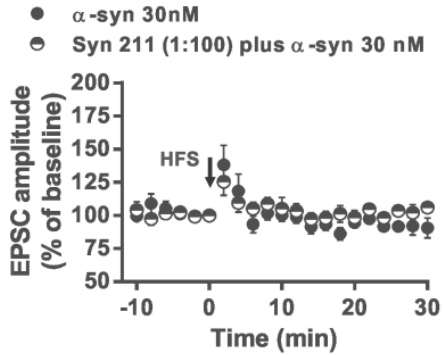

Supplementary Fig. 2 

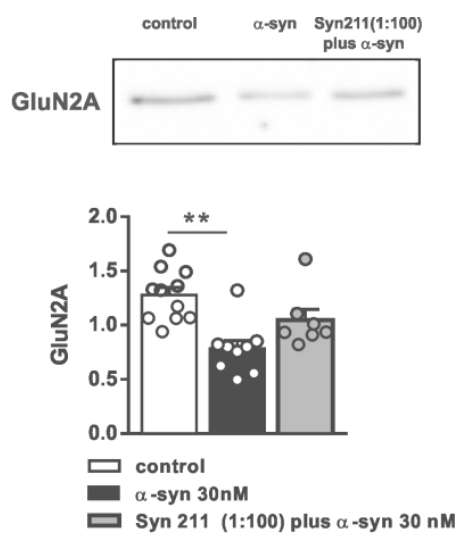

Supplementary Fig. 3 


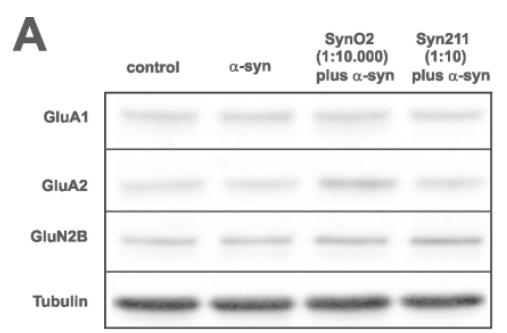

B

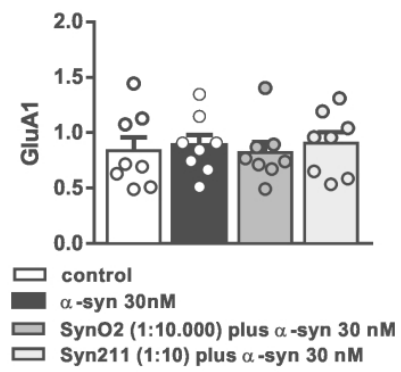

C

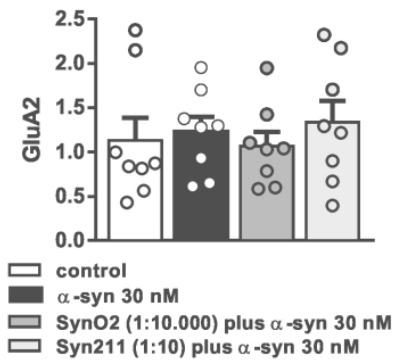

D

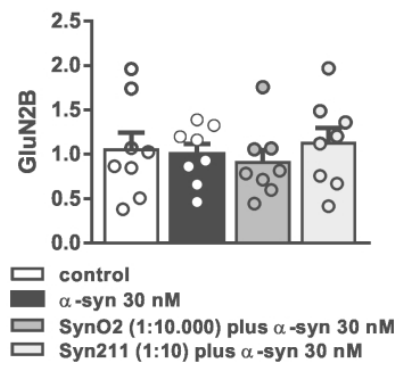

Supplementary Fig. 4 
Animals injected with $\alpha$-syn OLIGO

A $\alpha$-syn striatum

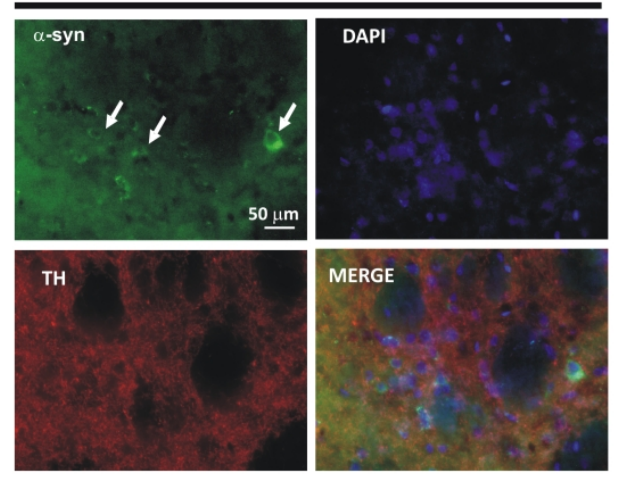

B control striatum

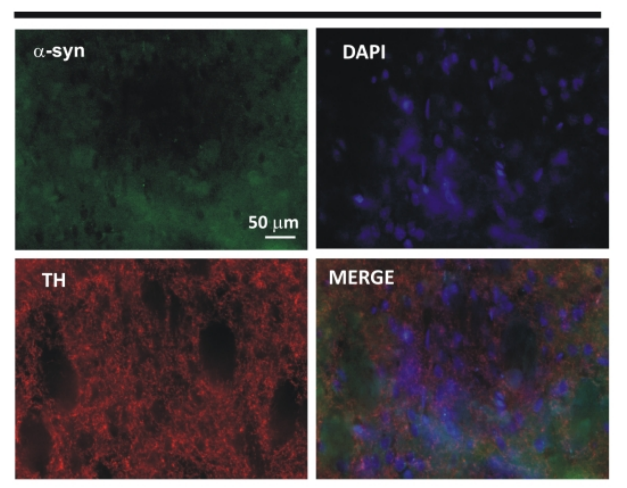

Supplementary Fig. 5 


\section{A Animals injected with $\alpha$-syn PPF}
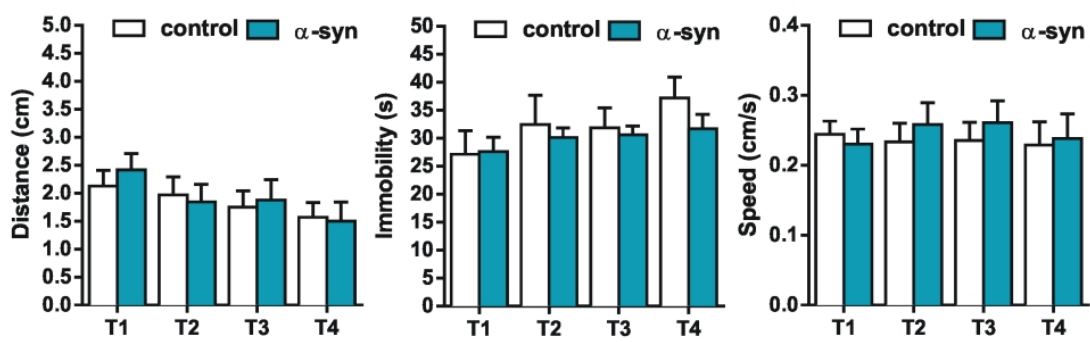

B

Animals injected with $\alpha$-syn OLIGO
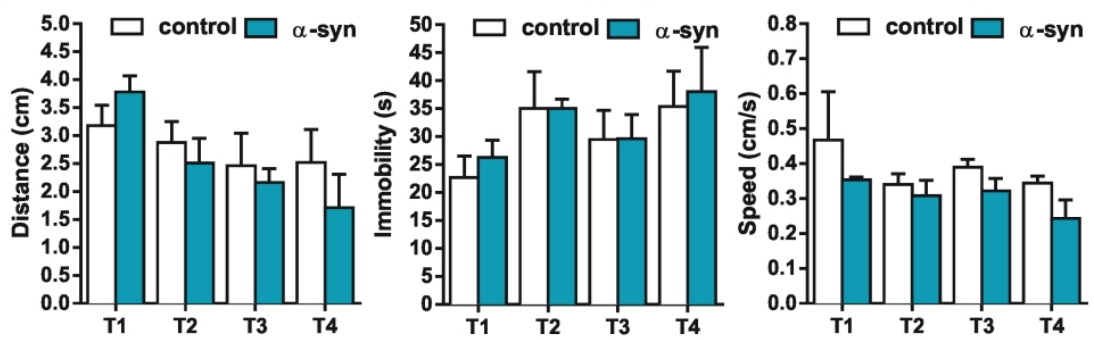

Supplementary Fig. 6 